Florida International University

FIU Digital Commons

FIU Electronic Theses and Dissertations

University Graduate School

$11-15-2013$

\title{
A Novel Signal Processing Method for Intraoperative Neurophysiological Monitoring in Spinal Surgeries
}

Krishnatej Vedala

Florida International University, kveda001@fiu.edu

DOI: $10.25148 /$ etd.FI13121209

Follow this and additional works at: https:// digitalcommons.fiu.edu/etd

Part of the Bioelectrical and Neuroengineering Commons, Biomedical Commons, Biomedical Devices and Instrumentation Commons, $\underline{\text { Neurosciences Commons, Other Neuroscience and }}$ Neurobiology Commons, Signal Processing Commons, and the Surgical Procedures, Operative Commons

\section{Recommended Citation}

Vedala, Krishnatej, "A Novel Signal Processing Method for Intraoperative Neurophysiological Monitoring in Spinal Surgeries" (2013). FIU Electronic Theses and Dissertations. 1038.

https://digitalcommons.fiu.edu/etd/1038 


\section{FLORIDA INTERNATIONAL UNIVERSITY \\ Miami, Florida}

\section{A NOVEL SIGNAL PROCESSING METHOD FOR INTRAOPERATIVE NEUROPHYSIOLOGICAL MONITORING IN SPINAL SURGERIES}

A dissertation submitted in partial fulfillment of the requirements for the degree of DOCTOR OF PHILOSOPHY

in

ELECTRICAL ENGINEERING

by

Krishnatej Vedala

2013 
To: Dean Amir Mirmiran

College of Engineering and Computing

This dissertation, written by Krishnatej Vedala, and entitled A Novel Signal Processing Method for Intraoperative Neurophysiological Monitoring in Spinal Surgeries, having been approved in respect to style and intellectual content, is referred to you for judgment.

We have read this dissertation and recommend that it be approved.

Armando Barreto

Jean Andrian

Naphtali Rishe

Malek Adjouadi, Major Professor

Date of Defense: November 15, 2013

The dissertation of Krishnatej Vedala is approved.

\begin{tabular}{r} 
Dean Amir Mirmiran \\
College of Engineering and Computing \\
\hline Dean Lakshmi N. Reddi \\
University Graduate School
\end{tabular}

Florida International University, 2013 
(C) Copyright 2013 by Krishnatej Vedala

All rights reserved. 


\section{DEDICATION}

I dedicate this dissertation to my wonderful parents and my sister. 


\section{ACKNOWLEDGMENTS}

This dissertation has been made possible by the collective support of my family, friends and mentors. I would like to thank my parents and my sister who are my greatest support system in life. Their unconditional love and support have kept me going through the hard times.

My mentor Dr. Malek Adjouadi has taught me a great deal in professional, personal and intellectual development. He has always had my best interest at heart and has provided me with every opportunity to grow. I am grateful for all the kindness and patience that he has shown me over the years. I would like to thank my gurus, Dr. Armando Barreto, Dr. Jean Andrian and Dr. Naphtalie Rishe, naming a few, whose interaction gave me new insights to my research work.

I would also like to thank our collaborators Dr. Ilker Yaylali and Dr. Melvin Ayala for their invaluable advice and directions. My thanks also go to my colleages at Center for Advanced Technology and Education (CATE) Dr. Mercedes Cabrerizo, Dr. Mohammed Goryawala, Dr. Changan Han, Dr. Jin Wang, Dr. Yu Chen and many more current and former members for their many useful suggestions.

I would also like to thank the members of the Electrical Engineering Department, Mr. Oscar Silveira, Ms. Pat Brammer and Ms. Ana Saenz and Ms. Maria Benincasa from the School of Engineering for the support throughout the course of my studies and the laughs we have shared.

I would like to express my special thanks to my friends around the globe. They have

come and gone but each one leaving a permanent impression in my life in a special 
way and taught me to live life to the fullest. Their support and good wishes have been the driving force in helping me successfully finish my dissertation. 


\title{
ABSTRACT OF THE DISSERTATION \\ A NOVEL SIGNAL PROCESSING METHOD FOR INTRAOPERATIVE \\ NEUROPHYSIOLOGICAL MONITORING IN SPINAL SURGERIES
}

\author{
by \\ Krishnatej Vedala \\ Florida International University, 2013 \\ Miami, Florida \\ Professor Malek Adjouadi, Major Professor
}

Intraoperative neurophysiologic monitoring (IONM) is an integral part of spinal surgeries and involves the recording of somatosensory evoked potentials (SSEP). However, clinical application of IONM still requires anywhere between 200 to 2000 trials to obtain an SSEP signal, which is excessive and introduces a significant delay to prevent potential neurological risks during surgery. The main objective of this dissertation is to develop a means to obtain the SSEP signal using a much reduced number of trials (20 trials or less) while still optimizing the effectiveness of the monitoring system. The preliminary research steps were to determine those characteristics that distinguish the SSEP with the ongoing brain activity. We first established that the brain activity is indeed quasi-stationary whereas an SSEP is expected to be identical every time a trial is recorded.

A novel algorithm is subsequently developed using Chebyshev time windowing for preconditioning of SSEP trials to retain the morphological characteristics of somatosensory evoked potentials (SSEP). This preconditioning was followed by the application of a principal component analysis (PCA)-based algorithm utilizing quasistationarity of EEG on 12 preconditioned trials. A unique Walsh transform operation was then used to identify the position of the SSEP event. An alarm is raised 
when there is a $10 \%$ time in latency deviation and/or $50 \%$ peak-to-peak amplitude deviation, as per the clinical requirements. The algorithm shows consistency in the results in monitoring SSEP in up to 6-hour surgical procedures even under this significantly reduced number of trials.

In this study, the analysis was performed on the data recorded in 29 patients who underwent surgery during which the posterior tibial nerve was stimulated and SSEP response was recorded from scalp EEG. This method is shown empirically to be more clinically viable than present day approaches. In all 29 cases, the algorithm took on an average 4sec to extract an SSEP signal, as compared to conventional methods, which take up to several minutes.

The monitoring process using the algorithm was successful and proved conclusive under the clinical constraints throughout the different surgical procedures with an accuracy of $91.5 \%$. Higher accuracy and faster execution time, observed in the present study, in determining the SSEP signals provided for a much improved and effective neurophysiological monitoring process. 


\section{TABLE OF CONTENTS}

CHAPTER

PAGE

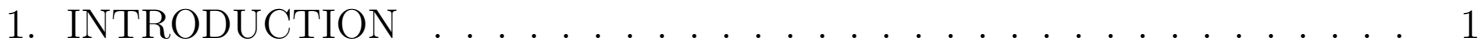

1.1 Intraoperative Monitoring . . . . . . . . . . . . . . . . . . 1

1.2 Motivation . . . . . . . . . . . . . . . . . . . 2

1.3 Hypotheses . . . . . . . . . . . . . . . . . . . . . . 3

1.4 Research Questions . . . . . . . . . . . . . . . . . . . . . . 4

1.5 Significance of the study . . . . . . . . . . . . . . . . 4

1.6 Literature Survey . . . . . . . . . . . . . . . . . . . . . 5

1.7 Database and Recordings $\ldots \ldots \ldots \ldots \ldots$

2. ALGORITHM VERSION 1 - EIGEN SPACE FILTERING . . . . . . . 10

2.1 Background . . . . . . . . . . . . . . . . . . . . . . 10

2.2 Structure of the algorithm . . . . . . . . . . . . . . . . . . 11

2.3 Implementation . . . . . . . . . . . . . . . . . . . . . 15

2.3.1 AMUSE algorithm - Noise components filtering . . . . . . . . . . 23

2.3.2 Walsh transform - Automated peak detection . . . . . . . . . . . 25

2.4 Implementation Results . . . . . . . . . . . . . . . . . . . 26

3. ALGORITHM VERSION 2 - TEMPORAL FILTERING . . . . . . . 37

3.1 Background . . . . . . . . . . . . . . . . . . . 37

3.2 Gaussian Template . . . . . . . . . . . . . . . . . . . . . 37

3.3 Chebyshev Filtering . . . . . . . . . . . . . . . . . 38

4. ALGORITHM VERSION 3 - COMBINATION OF EIGEN \& TEMPORAL FILTERING . . . . . . . . . . . . . . . . . . . 44

4.1 Merits of Pre-filtering . . . . . . . . . . . . . . . . . . . . 44

4.2 Implementation . . . . . . . . . . . . . . . . . . . . . . . . 44

4.2 .1 Data Acquisition . . . . . . . . . . . . . . . . . . . . . 44

4.2 .2 Pre-filtering or Pre-conditioning . . . . . . . . . . . . . . . . 47

4.2 .3 Eigen Space . . . . . . . . . . . . . . . . . . . . . . . . 47

4.2 .4 Reconstruction . . . . . . . . . . . . . . . . . . 51

4.2 .5 Information Extraction . . . . . . . . . . . . . . . 51

4.3 Results . . . . . . . . . . . . . . . . . . . . 51

4.3 .1 Implementation . . . . . . . . . . . . . . . . . . . . . . 51

4.3 .2 False Alarms . . . . . . . . . . . . . . . . . . . . . . . . . 54

5. CONCLUSIONS AND FUTURE WORK . . . . . . . . . . . 63

5.1 Conclusions from algorithm versions . . . . . . . . . . . . . 63

5.1 .1 Version $1 \ldots \ldots \ldots \ldots$

5.1 .2 Version $2 \ldots \ldots \ldots \ldots \ldots$

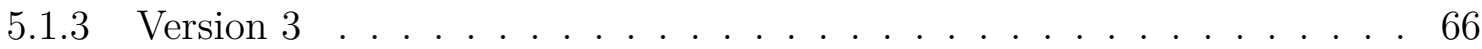


5.2 Discussion . . . . . . . . . . . . . . . . . . . . . . . . . 69

5.3 Future Study and Recommendations . . . . . . . . . . . . . . 71

5.3.1 Exhaustive study . . . . . . . . . . . . . . . . 71

5.3.2 Practical implementation . . . . . . . . . . . . . . . 71

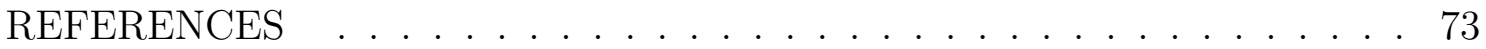

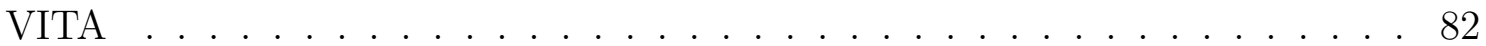




\section{LIST OF TABLES}

TABLE

PAGE

2.1 Results of analysis from the implementation of algorithm of version 1 . . 27

4.1 Patient details and algorithm version 3 accuracy results . . . . . . . . . 48

4.2 Summary of the results from the implementation of the proposed algorithm showing the average variability in the time latency and amplitude and the total number of false alarms raised for each patient. . . 57

4.3 Amplitude and time latency consistency for patient 10 from table 4.1 . . 59

5.1 Summary of results showing the average percentage error and percentage deviation ..................... 65 


\section{LIST OF FIGURES}

FIGURE

PAGE

1.1 Screen-shot of CASCADE IONM system displaying SSEP extracted from four bi-polar electrodes. . . . . . . . . . . . . . 8

2.1 Flowchart for the algorithm version 1 and the details of AMUSE algorithm are indicated by the arrow. . . . . . . . . . . . . . 12

2.2 Comparison between algorithm version 1 and clinical implmentations . . 16

2.2 (cont.) . . . . . . . . . . . . . . . . . 17

$2.3 \mathbf{Y}_{1}(n)$ - The ten components of the ten trials recorded, $\mathbf{X}_{1}(n)$, from the $\mathrm{C}_{\mathrm{Z}}-\mathrm{F}_{\mathrm{Z}}$ channel and decomposed using AMUSE algorithm. The $\mathrm{X}$-axis represents the sampling time intervals. . . . . . . . . . . . 19

2.4 PSD of two components from fig. 2.3 . . . . . . . . . . . . . . 20

2.4 (cont.) . . . . . . . . . . . . . . . . . . . . 21

2.5 Reconstruction of SSEP from high frequency and low frequency compo-

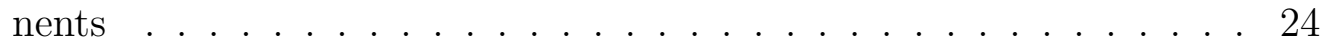

2.6 Significance of removing the low-frequency components . . . . . . . . . . 33

2.7 Application of Walsh transform to identify the position of the SSEP . . 34

2.8 Automated detection of evoked response from 10 trials. . . . . . . . . . 35

2.9 Consistency in detecting P37 and N45 peak latencies from the $\mathrm{C}_{\mathrm{Z}}-\mathrm{F}_{\mathrm{Z}}$ recording of subject 20 with the peak latencies (algorithm vs. clinical) as given in table 2.1d. . . . . . . . . . . . . . . . . . . . . . 36

3.1 Outline of the SSEP recording timeline as compared with the clinical monitoring. By the time one clinical SSEP is obtained, the algorithm version 2 obtained 10 SSEP signals.

3.2 Comparison of signals before and after applying Chebyshev filtering with the actual average signal. . . . . . . . . . . . . . . . . . . 42

3.3 The signal to noise ratio (SNR) comparison for raw signals and after. SNR values are provided for the two cases when 12 trials are used and for 200 raw trials. . . . . . . . . . . . . . . . . . . 43

4.1 Flowchart for the complete algorithm version 3. . . . . . . . . . . 45

4.2 A set of twelve successive unrejected recordings for the patient 7 in table 4.1. . . . . . . . . . . . . . . . . . . . . 46

4.3 The components of a set of 12 raw sweeps obtained using the AMUSE algorithm . . . . . . . . . . . . . . . . . . . 49

4.4 The components of a set of 12 pre-conditioned sweeps obtained using the AMUSE algorithm . . . . . . . . . . . . . . . . . . 50

4.5 A comparison of the components and the extracted SSEP from fig. 4.3 (dotted lines) and fig. 4.4 (solid lines). . . . . . . . . . . . . . . . 52

4.6 Comparison of SSEP extracted by the algorithm using only 12 trials with that obtained using the traditional method of averaging of 113 trials. 
4.7 Outline of the SSEP recording timeline as compared with the clinical monitoring. By the time one clinical SSEP is obtained, the algorithm version 3 obtaining 12 SSEP signals. . . . . . . . . . . . . 54

4.8 Sequence of extracted SSEPs of $100 \mathrm{~ms}$ throughout the surgery. . . . . . 56

4.9 The signal to noise ratio (SNR) comparison for clinical SSEP signals and post-processed signals. SNR values are provided for the two cases when 12 trials are used and for 200 raw trials. . . . . . . . . . . . . . 60

4.10 Comparison of a false positive SSEP signals as detected due to high amplitude variation for patient 8 in table $4.1 \mathrm{C}_{3}-\mathrm{C}_{4}$ montage at 23min through the procedure with the baseline SSEP. . . . . . . . 61

4.11 The baseline signal and tSSEP signals at different times shown for com-

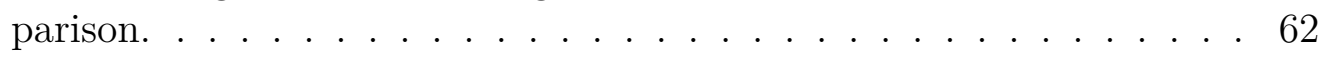




\section{SYMBOLS AND ABBREVIATIONS}

$\begin{array}{ll}\text { AMUSE } & \text { Algorithm for multiple signal extraction } \\ \text { AVF } & \text { Arteriovenous fistula } \\ \text { DCT } & \text { Discrete cosine transform } \\ \text { EEG } & \text { Electroencephalogram } \\ \text { FFT } & \text { Fast Fourier transform } \\ \text { FIR } & \text { Finite impulse response } \\ \text { IFFT } & \text { Inverse FFT } \\ \text { IIR } & \text { Infinite impulse response } \\ \text { IOM } & \text { Intraoperative monitoring } \\ \text { IONM } & \text { Intraoperative neurophysiological monitoring } \\ \text { LPF } & \text { Low pass filter } \\ \text { MEP } & \text { Motor evoked potentials } \\ \text { N45 } & \text { Negative peak of SSEP at 45ms } \\ \text { P37 } & \text { Positive peak of SSEP at 37ms } \\ \text { PCA } & \text { Principal component analysis } \\ \text { SNR } & \text { Signal to noise ratio } \\ \text { SEP } & \text { Sensory evoked potentials } \\ \text { SSEP } & \text { Somatosensory evoked potentials } \\ \text { SVD } & \text { Singular value decomposition } \\ \text { TLIF } & \text { Transforaminal lumbar interbody fusion surgery } \\ \text { tSSEP } & \text { Tibial nerve SSEP }\end{array}$




\section{CHAPTER 1 \\ INTRODUCTION}

\subsection{Intraoperative Monitoring}

Intraoperative monitoring (IOM) is an important protocol that clinicians adhere to during surgeries. The patient undergoing a surgery is continuously monitored for a variety of physiological processes so as to make sure that the surgery does not lead to unanticipated and potential long term changes. The IOM protocols were developed and form standard protocols in procedures when the spine, brain and peripheral nerves are at risk. Examples of IOM include the following:

(a) Intraoperative fetal monitoring during nonobstetric surgery in pregnancy (Kilpatrick et al., 2010)

(b) Coronary sinus lactate assay for metabolic monitoring of heart (Crittenden, 2001)

(c) Sensory evoked potentials for functional integrity of sensory pathways. (Grundy, 1983)

Iatrogenic spinal cord injury is the most feared complication of scoliosis surgery. The use of somatosensory-evoked potentials (SSEPs) as a monitoring tool during neurosurgical procedures has been reported widely in literature dating as far back as the late 1940s (Dawson, 1947).SSEPs are nowadays routinely used for monitoring the function of the spinal cord in procedures when the spine, brain and peripheral nerves are at risk. They have been utilized in major studies that have reported procedures affecting the spine such as deformity correction, spinal fracture repair 
and tumor removal (Jones et al., 1983; Nash et al., 1977; Nuwer and Dawson, 1984); procedures affecting the brain such as aneurysm repair and carotid endarterectomy (Friedman et al., 1991; Hargadine and Snyder, 1982; Lam et al., 1991); and procedures involving the peripheral nerves (Mahla et al., 1984; Nercessian et al., 1989; Porter et al., 1989). They are also used for the identification of the sensory portion of the sensorimotor cortex (Celesia, 1979). SSEPs are also widely used for intraoperative neurophysiological monitoring (IONM) in surgeries for scoliosis (Pastorelli et al., 2011; Schwartz et al., 2007), pedicle-screw placement procedures (Jou et al., 2003) and spinal cord related surgical procedures (Dinner et al., 1986; McGarry et al., 1984; Nuwer et al., 1995; Deletis and Sala, 2008; Deletis, 2007).

\subsection{Motivation}

Somatosensory evoked potentials (SSEP) monitoring is a valuable tool for medical diagnostics and surgical purposes (Dinner et al., 1986; Strahm et al., 2003; Khan et al., 2006; Epstein et al., 1993; Toleikis, 2005). These electroencephalography (EEG)-based signals are obtained through external stimulus applied to a sensory organ such as the tibial nerve, and are identified by a positive peak followed by a negative peak with a specific time range and amplitude.

The SSEP are characterized by a fixed time difference between the application of the stimulus and the occurrence of these two peaks (i.e., time latency) and the peak-to-peak amplitude of the signal. The peaks are identified by an alphabet (P or $\mathrm{N}$ ) indicating positive or negative peak followed by a number indicating the peak latency in milliseconds. For a healthy average human, the tibial nerve SSEP peaks are P37 and N45 i.e., a positive peak at $37 \mathrm{~ms}$ and a negative peak at $47 \mathrm{~ms}$. In the recorded EEG signals, the SSEP is the required signal and all other superimposed 
signals are considered as noise. The signal to noise ratio (SNR) of SSEP is very low because of other signals superimposed on the EEG, and this has been the major barrier for their study. It was Dr. Dawson who first introduced the potential application of SSEP (Dawson, 1947) and also realized the difficulty in extracting them from the EEG, and hence his approach of averaging the signals in time has proven very effective and remains the predominant practice. This method, however,

improves the SNR by a factor of $\sqrt{N}$ and hence requires a large number of trials to be averaged to obtain the desired SSEP, typically ranging from few hundreds up to 3000 trials. The stimulus is typically provided at a rate of $3 \mathrm{~Hz}$ (Society, 2006) and hence the time required before obtaining the SSEP could be quite large. Thus, a robust method of recording and monitoring SSEP with a minimum number of trials, although challenging, provides valuable information for the clinicians during surgery.

\subsection{Hypotheses}

The following are the main hypotheses that are assumed for intra-operative monitoring within the research context of this dissertation:

(a) The recorded EEG signals are wide sense ergodic processes with zero-mean white Gaussian noise.

(b) The eigen system components, of the recorded EEG signals, obtained using the algorithm are mutually independent.

(c) The SSEP obtained from the first recording, called the baseline signal, is the ideal SSEP and is clinically assumed to remain constant for that patient throughout the surgical procedure. 
(d) Time latency deviation by no more than $10 \%$ from the baseline, and peak-topeak amplitude deviations of no more than 50\% from the baseline are considered standard safeguards.

\subsection{Research Questions}

Research questions that are posed on the basis of the aforementioned hypotheses include:

(a) What characterizes an evoked potential from a regular action potential?

(b) Why do conventional signal processing algorithms fail to extract SSEP from an EEG recording?

(c) In conventional PCA analysis, the components corresponding to the higher eigenvalues are the noise introducing components. Would this conventional wisdom work for SSEP signals as well? Does this signify the extremely low signal-to-noise ratio (SNR) of the SSEP?

(d) On what factors is the optimum number of signals for PCA analysis based upon?

(e) If the SSEP being recorded is the result of a pulse stimulus, can the nerve conduction and electrical pathway be modeled as the step response of an unknown linear or non-linear electrical system?

\subsection{Significance of the study}

The research is significant for two specific reasons:

(a) Higher prospects for detecting SSEP signals with high accuracy and consistency throughout the entire surgical procedure and with the ability of using a 
much-reduced optimal number of trials will provide the surgeons timely valuable information to guide their course of action during the surgical procedures while ensuring a more effective monitoring process.

(b) Provide as a consequence enhancements to intraoperative neurophysiological monitoring since the SSEP signals can now be acquired faster for closer scrutiny of neurological function during spinal surgery and thus reduce the likelihood of post-operative physiological complications.

\subsection{Literature Survey}

Although effective, the averaging technique has a major drawback in that it requires a significant amount of trials to be collected in order to generate a realistic SSEP, which is not practical in terms of the monitoring time required (Hussain, 2008). As a result of this unyielding problem, various efforts have been made to reduce the number of trials. Efforts to reduce the number of trials have used techniques such as parametric decomposition (Bai et al., 2001), Bayesian analysis (Truccolo et al., 2003) and digital filters (Friedman et al., 1991). Another study reported the use of amplitude-modulated stimulus while performing steady-state analysis on the recorded signals (Noss et al., 1996). Also, the latency as the time difference between successive trials was used for noise removal (Kong and Oiu, 2001).

More recent advances using functional source separation of SSEP signals have been utilized to provide information about underlying EEG characteristics that can be used for SSEP detection (Porcaro et al., 2009). Phase-based techniques have also been used to successfully reduce the number of trials to 200 (Simpson et al., 2000). A more recent approach using neural networks was used to classify auditory-evoked 
potentials and to classify anesthetic states but relied on 1000 trials (Zhang et al., 2001).

Some studies have also shown detection of SSEP from as low as a single trial but such techniques are greatly affected by the recording noise (Hu et al., 2011a; Nishida et al., 1993; Turetsky et al., 1989). Other studies have shown a comparison of various blind source separation techniques for SSEP detection (Liu et al., 2011). These often rely on correlation measures to evaluate the extracted SSEP from the baseline or true SSEP and do contend with the amount of noise present in the EEG recordings.

Blind source separation does not guarantee extraction of the SSEP by one of its components. The understanding of the fact that SSEP is one of the sources resulting to the EEG and the remaining finite number of sources can be attributed to noise. It then becomes tempting to develop and apply blind source separation (BSS) algorithms to extract the useful source, the SSEP. Here, stress must be placed on the fact that all the sources of the EEG change in time and amplitude. It then follows that none of PCA or ICA components can on their own give complete information of the SSEP. BSS has been proved quite effective in extracting auditory evoked potentials using higher order correlations. This was facilitated by the proximity of stimulus source to the brain.

However, the sources of SSEP are the distal ends of tibial and ulnar nerves and the recorded signals also have the disturbance arising from the ongoing surgical procedure. Previous studies on SSEP using PCA using a multitude of trials always found that the SSEP waveform characteristics are always distributed among more than one component. Each eigenvector contributes to a certain percentage of variance in the data. Hence, different PCA and ICA methods extract different information 
from the SSEP but not a complete SSEP unless a large number of trials are used for analysis and thus affecting the goal of the study.

Standard algorithms often consider time-amplitude variations between individual trials and common features between the individual signals. The later fact led to the use of the principal component analysis (PCA) for estimating components associated with noise (Glaser and Ruchkin, 1976; Moore, 1981; Regan, 1990; Suter, 1970). The PCA is based on eigen-decomposition of the raw SSEP signal matrix. A modified version of PCA-based signal decomposition technique named Algorithm for Multiple Signal Extraction (AMUSE) (Crespo-Garcia et al., 2008; Tong et al., 1990) showed great potential at reducing the number of trials to the ten noise-free trials of the first twenty trials only.

\subsection{Database and Recordings}

All data for all the patients that were considered as part of the study in this dissertation came de-identified from recorded surgeries performed at Oregon Health and science University (OHSU) hospital for over a period of one year from September 2010 to November 2011. The data was collected initially from 16 patients with just one set of recordings and thereafter 12 more patients with continuous recordings throughout the surgeries were obtained. The clinical monitoring was performed using CASCADE IONM as shown in fig. 1.1. The tSSEP were chosen because of their wide study and reliability as they lie farthest from the brain and provide the longest

path for the SSEP (Fukuda et al., 2007; Kany and Treede, 1997; Sako et al., 1998; Terada et al., 2009; van de Wassenberg et al., 2008).

Stimuli of intensity $45 \mathrm{~mA}$ were applied to the posterior tibial nerve with a pulse 


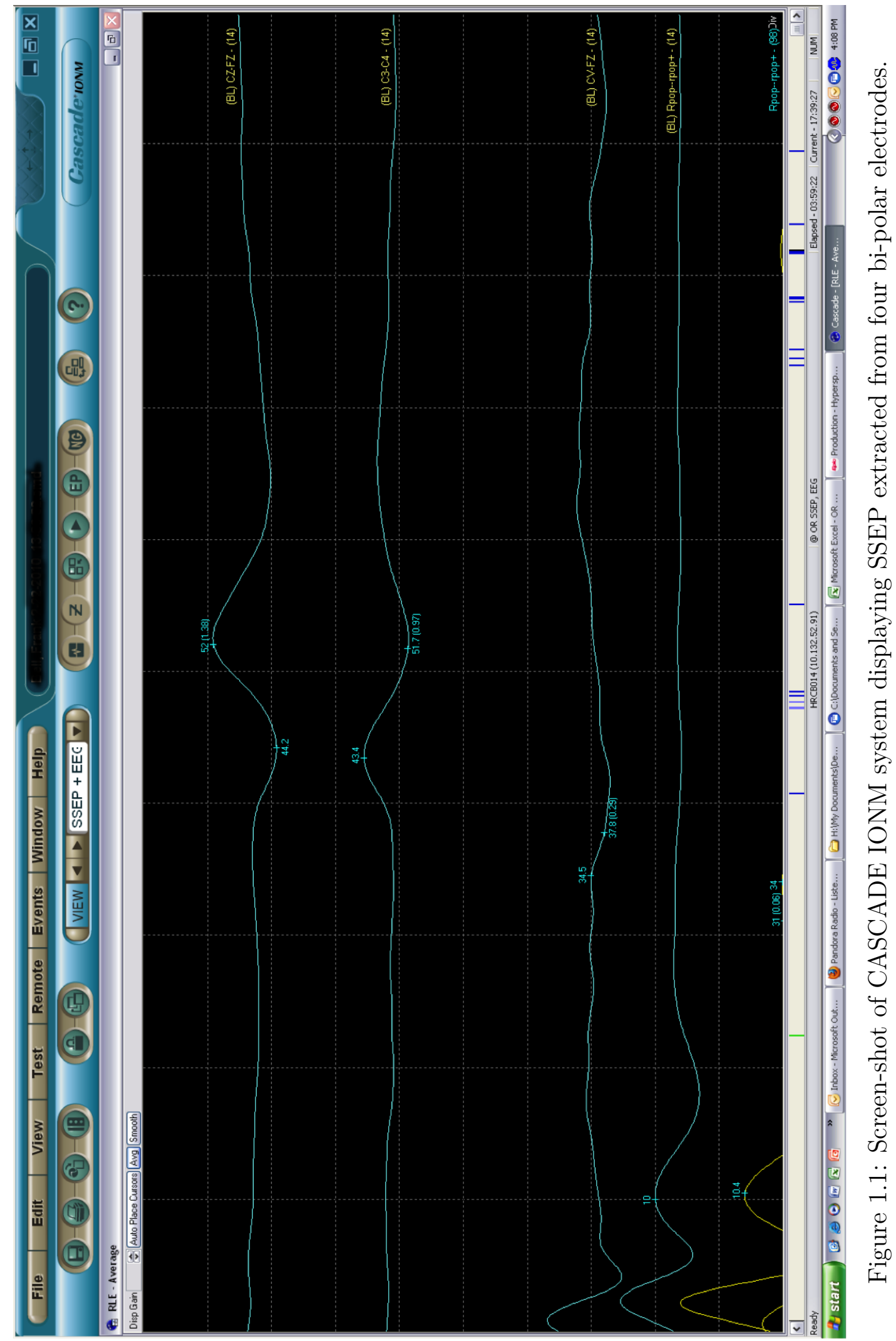


repetition rate of 3.1 per second and the evoked potentials were recorded from the scalp following the international 10/20 system. Two bipolar recordings viz., the $\mathrm{C}_{3}{ }^{-}$ $\mathrm{C}_{4}$ and $\mathrm{C}_{\mathrm{Z}}-\mathrm{F}_{\mathrm{Z}}$ were recorded and digitized at $6400 \mathrm{~Hz}$ sampling rate for a duration of $100 \mathrm{~ms}$ ensuing a total of 640 samples and band-limited between $30 \mathrm{~Hz}$ and $500 \mathrm{~Hz}$. The surgical procedures lasted for 1.5 to 6 hours during which time the patients were monitored continuously. All the surgeries were successful without the raise of any alarms in the tSSEP and the primary goal was to observe if the proposed algorithm was able to correctly validate the results. The surgical cases presented have been successful and no alarm was raised during the procedures to prove the consistency of the algorithm.

Consistency in positive and negative peaks and the peak-to-peak amplitude are the main characteristics of the SSEP sought in the monitoring process for any patient undergoing surgery. In a tSSEP signal, the typical time latency for a positive peak is $37 \mathrm{~ms}$ and that of a negative peak is $45 \mathrm{~ms}$ (referred to as P37 and N45, respectively). (Pastorelli et al., 2011; Nuwer, 2008). During a surgical procedure, these values obtained from the first recording are termed as the baseline values and are clinically expected to remain consistent throughout the procedure. Any research endeavor involving SSEP would have to extract and then effectively and accurately monitor such main characteristics during the entire procedure, and if for any unforeseen event, such monitoring must also include timely warning for any cause for alarm. 


\section{CHAPTER 2}

\section{ALGORITHM VERSION 1 - EIGEN SPACE FILTERING}

\section{$2.1 \quad$ Background}

The approach that was first undertaken in this study reduces this average to an optimal number of ten trials using an eigen-decomposition technique coupled with a unique Walsh operator to pinpoint the position of maximum amplitude, which served as an indicator of the presence of the SSEP peaks. A measure of caution was taken, in that a thorough mathematical assessment of the eigen components was performed at the onset to remove any trial that was fraught with noise in order not to burden the averaging process with the intention not to exceed 10 trials as

a maximum. In the clinical cases involved in this study, with a stimulus rate of $3.1 \mathrm{~Hz}$, using 200 to 500 trials, the time required to record the trials varied anywhere between 70 s to $3.2 \mathrm{~min}$.

Standard algorithms often consider time-amplitude variations between individual trials and common features between the individual signals. The latter fact prompted the use of principal component analysis (PCA) for estimating components associated with noise and the SSEP (Suter, 1970; Glaser and Ruchkin, 1976; Moore, 1981; Regan, 1990). The PCA is an analysis that is based on eigen-decomposition of a signal matrix. A modified version of PCA based signal decomposition technique named Algorithm for Multiple Signal Extraction (AMUSE) (Crespo-Garcia et al., 2008; Tong et al., 1990) was implemented to reduce the number of trials to 10. In retrospect, the AMUSE algorithm is equivalent to cascading two PCA systems (Tong et al., 1991), with the following basic assumptions: 
1. Data is a set of zero-mean wide sense ergodic process, the components of which are mutually independent.

2. Noise in the data is assumed zero-mean white Gaussian noise.

To satisfy the first condition, however, the arrangement of the recorded trials was verified to be Toeplitz matrix and thus implying ergodicity (Wirfalt and Jansson, 2010).

Walsh transform was implemented such as to automate the latency detection after obtaining the SSEP signal. The broad scope of applicability of the Walsh transform yielded, as examples, excellent results in (a) extracting stereo features to recover depth information in 2-D images (Adjouadi and Candocia, 1994; Adjouadi et al., 1996; Candocia and Adjouadi, 1997) and (b) in detecting interictal spikes in EEG data as means to detect seizures in pediatric epilepsy (Adjouadi et al., 2004, 2005;

Tito et al., 2010). In this SSEP application, a second order Walsh operator was found to be extremely effective in localizing the SSEP under only 10 trials even when the morphology of this signal is not yet quite similar as that of the so-called true morphology obtained with a much larger number of trials (200 to 500).

\subsection{Structure of the algorithm}

The structure of the proposed algorithm is illustrated in fig. 2.1. This figure contains the main mathematical derivations that were used with a brief description of what each step performs.

1. The original data matrix, which certainly includes noisy signals, is generated using recorded SSEP signals during successive trials from the same bipolar 


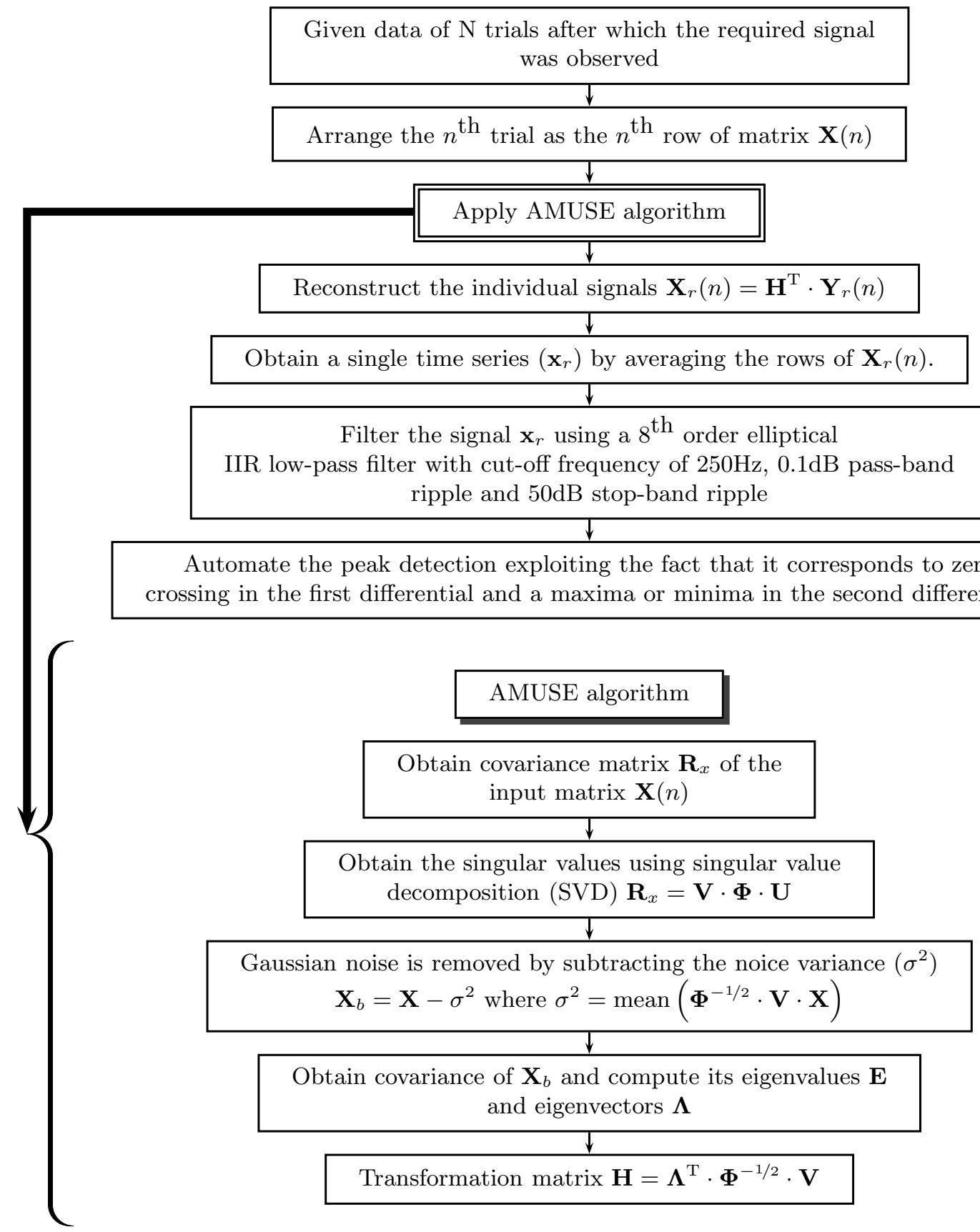

Figure 2.1: Flowchart for the algorithm version 1 and the details of AMUSE algorithm are indicated by the arrow. 
recording channel spanning the rows

$$
\mathbf{X}=\left[\begin{array}{cccc}
x_{1}(1) & x_{2}(1) & \cdots & x_{m}(1) \\
x_{1}(2) & x_{2}(1) & \cdots & x_{m}(1) \\
\vdots & \vdots & \ddots & \vdots \\
x_{1}(n) & x_{2}(n) & \cdots & x_{m}(n)
\end{array}\right]=\left[\begin{array}{c}
\mathbf{x}(1)^{T} \\
\mathbf{x}(2)^{T} \\
\vdots \\
\mathbf{x}(n)^{T}
\end{array}\right]
$$

where $\mathbf{X}(n)=\left[\begin{array}{llllll}x_{1}(n) & x_{2}(n) & \cdots & x_{m}(n) & \cdots & x_{M}(n)\end{array}\right]^{T}$ is the $\mathrm{n}^{\text {th }}$ trial data, $\mathbf{x}(n)$ is the vector of $\mathrm{n}^{\text {th }}$ trial and $x_{m}(n)$ is the $\mathrm{m}^{\text {th }}$ sample from $\mathrm{n}^{\text {th }}$ trial data.

2. The AMUSE algorithm (Tong et al., 1991; Crespo-Garcia et al., 2008) similar to the principal component analysis is applied on the matrix $\mathbf{X}$ following the steps below:

(a) Compute the $(\mathrm{N} \times \mathrm{N})$ covariance matrix:

$$
\mathbf{R}_{x}=\mathbf{X} \cdot \mathbf{X}^{T}
$$

(b) Determine the singular values $\boldsymbol{\Phi}$ of $\mathbf{R}_{x}$ using singular value decomposition (SVD) technique giving three matrices; $\mathbf{U}$ is a unitary matrix, $\mathbf{V}$ is a diagonal matrix for transformation and $\boldsymbol{\Phi}$ is the required matrix:

$$
\mathbf{R}_{x}=\mathbf{V} \cdot \boldsymbol{\Phi} \cdot \mathbf{U}
$$

(c) Remove the Gaussian noise components by subtracting the noise variance 
estimated as the mean of the singular matrix with the equation eq. (2.4)

$$
\sigma^{2}=\operatorname{mean}\left(\boldsymbol{\Phi}^{-\frac{1}{2}} \cdot \mathbf{V} \cdot \mathbf{X}\right)
$$

such that

$$
\mathbf{X}_{b}=\mathbf{X}-\sigma^{2}
$$

(d) Determine the covariance of $\mathbf{X}_{b}$ and further decompose it to find its eigenvalues and the corresponding eigenvectors $\Lambda$ to be used in step (e). This step ensures that all the singular values are distinct.

(e) Obtain the transformation matrix

$$
\mathbf{H}=\boldsymbol{\Lambda}^{T} \cdot \boldsymbol{\Phi}^{-\frac{1}{2}} \cdot \mathbf{V}
$$

(f) Determine the independent components as

$$
\mathbf{Y}(n)=\mathbf{H} \cdot \mathbf{X}
$$

3. Individual components were then studied and those that had the difference between first two consecutive frequency peaks less than $-30 \mathrm{~dB} / \mathrm{Hz}$ were removed. To remove a component, the corresponding row of $\mathbf{Y}(n)$ was replaced with zeros and a new matrix $\mathbf{Y}_{r}(n)$ was consequently constructed.

4. New individual signals as rows of $\mathrm{X}$ were then retrieved from the matrix $\mathbf{Y}(n)$ obtained in step 2-(e) above using the equation:

$$
\mathbf{X}_{r}(n)=\mathbf{H}^{T} \cdot \mathbf{Y}_{r}(n)
$$


5. Arithmetic mean was computed across each column to obtain a single time varying signal and was passed through a $250 \mathrm{~Hz}$ low pass filter (LPF), for experimental reasons that are detailed in the implementation section.

6. Detection of the peak that coincides with the occurrence of the SSEP was automated using the Walsh transformation method (Weide et al., 1978; Smith, 1981; Adjouadi et al., 2004, 2005) to indicate the evoked potential response.

Thus, implementing the algorithm on its own assuming the number of sources equal to the number of trials and a suitable noise variance $\sigma$ will remove the white Gaussian noise from the signals. Additional improvement can be obtained if the known noise components in the matrix are removed. Since the eigenvalues are arranged in descending order of their magnitude, the estimated components are arranged according to increasing statistical variance; often referred to as signal complexity implying addition of more frequency components. The second condition is automatically satisfied since the brain activity is a random process.

\subsection{Implementation}

As an illustrative example, fig. 2.2 shows comparative results of the algorithm using 10 trials for a subjects 16 and 20, shown here as illustrative examples, in contrast with the results obtained using the conventional method with 200 trials. MATLAB® programs were created by the authors that take the raw signal vector and the possible number of components as input and returns the estimated components, the transformation matrix, and reconstructed signals as the output. Once the components are estimated, filtering is used to remove unwarranted components. 


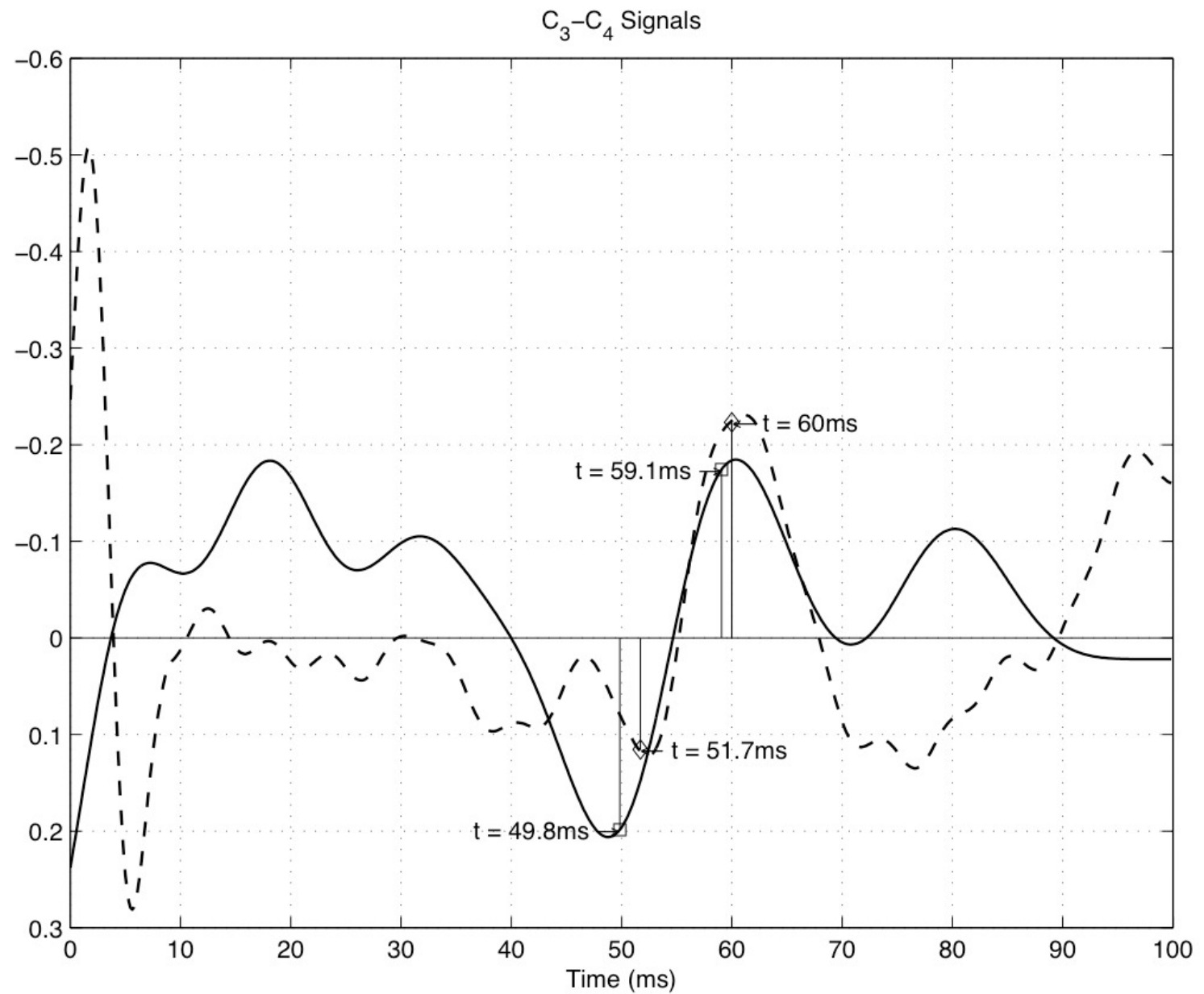

(a) Subject 16: A close approximation and a very similar morphology

Figure 2.2: Illustration showing a comparison between the results of the algorithm using 10 trials (solid line) and the clinical data using 200 trials (dotted line). Time instances shown to the left of the markers are the locations where the evoked potential was detected using the Walsh transformation method on $\mathrm{C}_{3}-\mathrm{C}_{4}$ signal. The time values adjecent the markers are the time instances of the SSEP selected by clinical experts. 


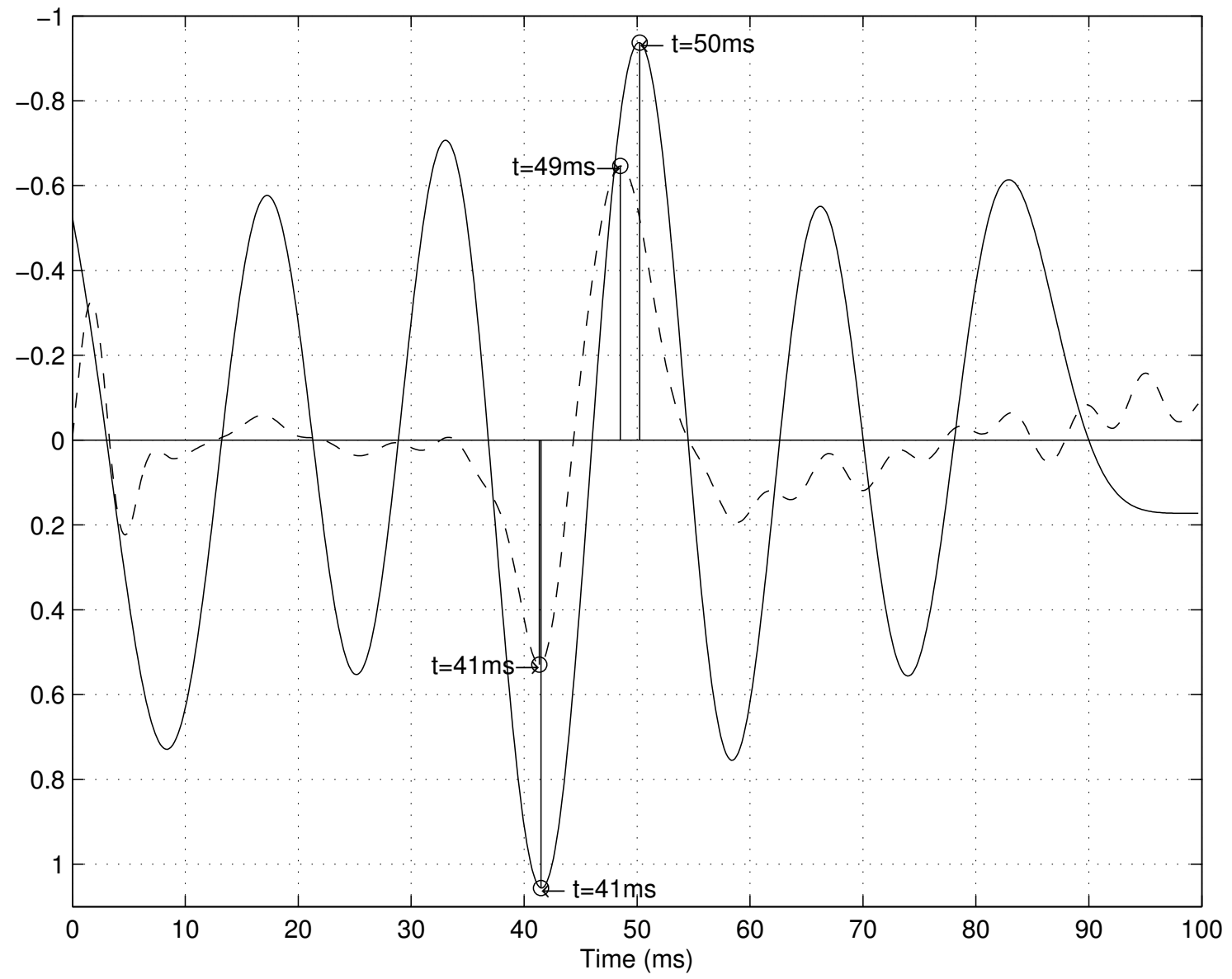

(b) Subject 20: A very close approximation with very ambiguous morphology

Figure 2.2: (cont.) 
Note the small discrepancies that exist between the markers for the negative and positive peaks as detected through 10 trials only in contrast to those of the signal obtained using 200 trials. These results are considered significant although the morphologies of the two signals are still quite different.

This particular study involved initially 16 subjects with the objective to estimate the location of the SSEP event using only 10 trials in comparison to the locations provided by clinical experts using 200 to 500 trials; and four other subjects were later included in the study with recordings provided at different stages of their respective surgical procedures. These later datasets were assessed to ascertain consistency and reproducibility of the results. For the recording process, two bipolar channels $\mathrm{C}_{3}-\mathrm{C}_{4}$ and $\mathrm{C}_{\mathrm{Z}}-\mathrm{F}_{\mathrm{Z}}$ were used to record the desired signals. For these patients, the SSEP signals were recorded by applying stimuli of intensity $45 \mathrm{~mA}$ and pulse duration of $200 \mu$ s at posterior tibial nerve of the right leg with a $3.1 \mathrm{~Hz}$ repetition rate. The positive terminal of stimulus is connected to the distal end and the negative terminal to the proximal end of the tibial nerve. The amplifier gain was set to 10 and a $25 \mu \mathrm{V}$ trial rejection threshold is used. The data was recorded at $6400 \mathrm{~Hz}$ sampling rate for duration of $100 \mathrm{~ms}$ and with the $60 \mathrm{~Hz}$ external interference component removed, yielding 640 samples per signal. The raw trial signals are band-limited from $10 \mathrm{~Hz}$ to $1000 \mathrm{~Hz}$ and the clinical average between $30 \mathrm{~Hz}$ and $500 \mathrm{~Hz}$. For illustration purposes, the 10 resulting independent components in one of the studies are shown in fig. 2.3 with similar results obtained for the other recording channel.

From fig. 2.3, it is evident that the higher eigenvectors contain the larger amount of background EEG components. These characteristic low frequency components were observed in data obtained from all the test subjects and varied from $6 \mathrm{~Hz}$ to $25 \mathrm{~Hz}$, while the high frequency components were as high as $418 \mathrm{~Hz}$. The frequency 

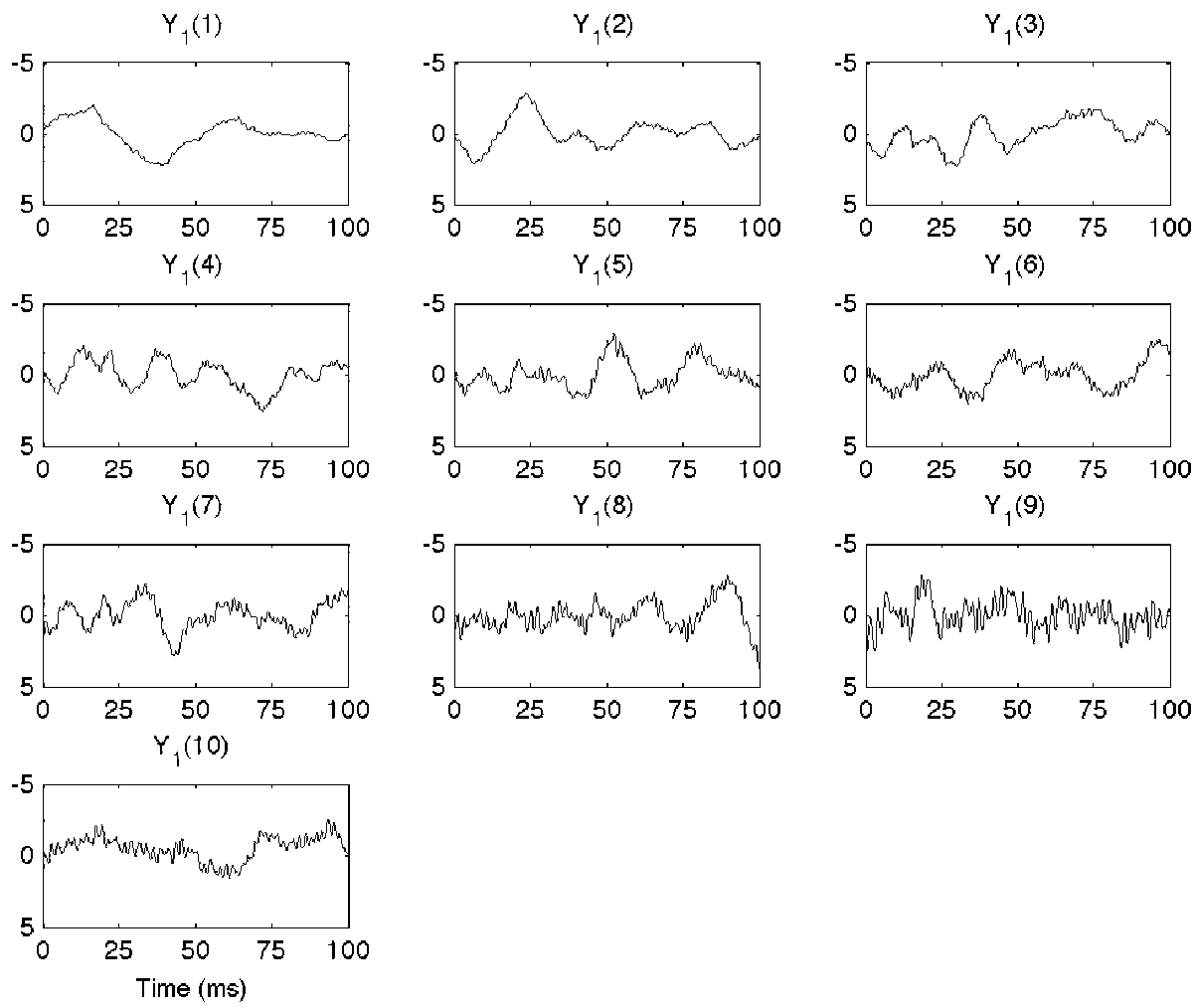

Figure 2.3: $\mathbf{Y}_{1}(n)$ - The ten components of the ten trials recorded, $\mathbf{X}_{1}(n)$, from the $\mathrm{C}_{\mathrm{Z}}-\mathrm{F}_{\mathrm{Z}}$ channel and decomposed using AMUSE algorithm. The $\mathrm{X}$-axis represents the sampling time intervals. 


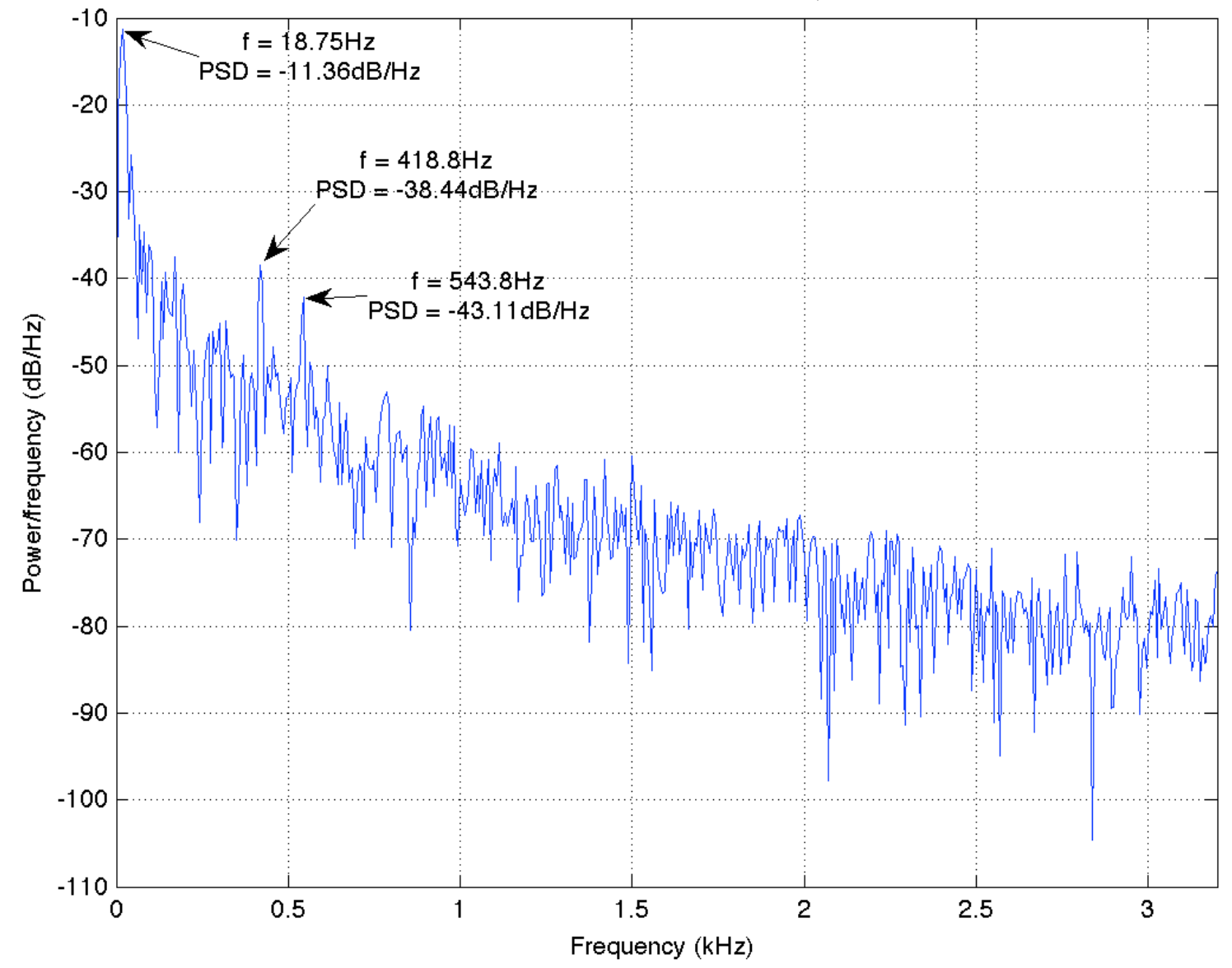

(a) PSD of $Y_{1}(1)$, obtained from the largest eigenvalue

Figure 2.4: PSD of two components from fig. 2.3: The symbol $f$ indicates the frequency and 'PSD' indicates the PSD at the corresponding frequency. PSD was estimated using periodogram estimation method (Stoica and Moses, 1997). 


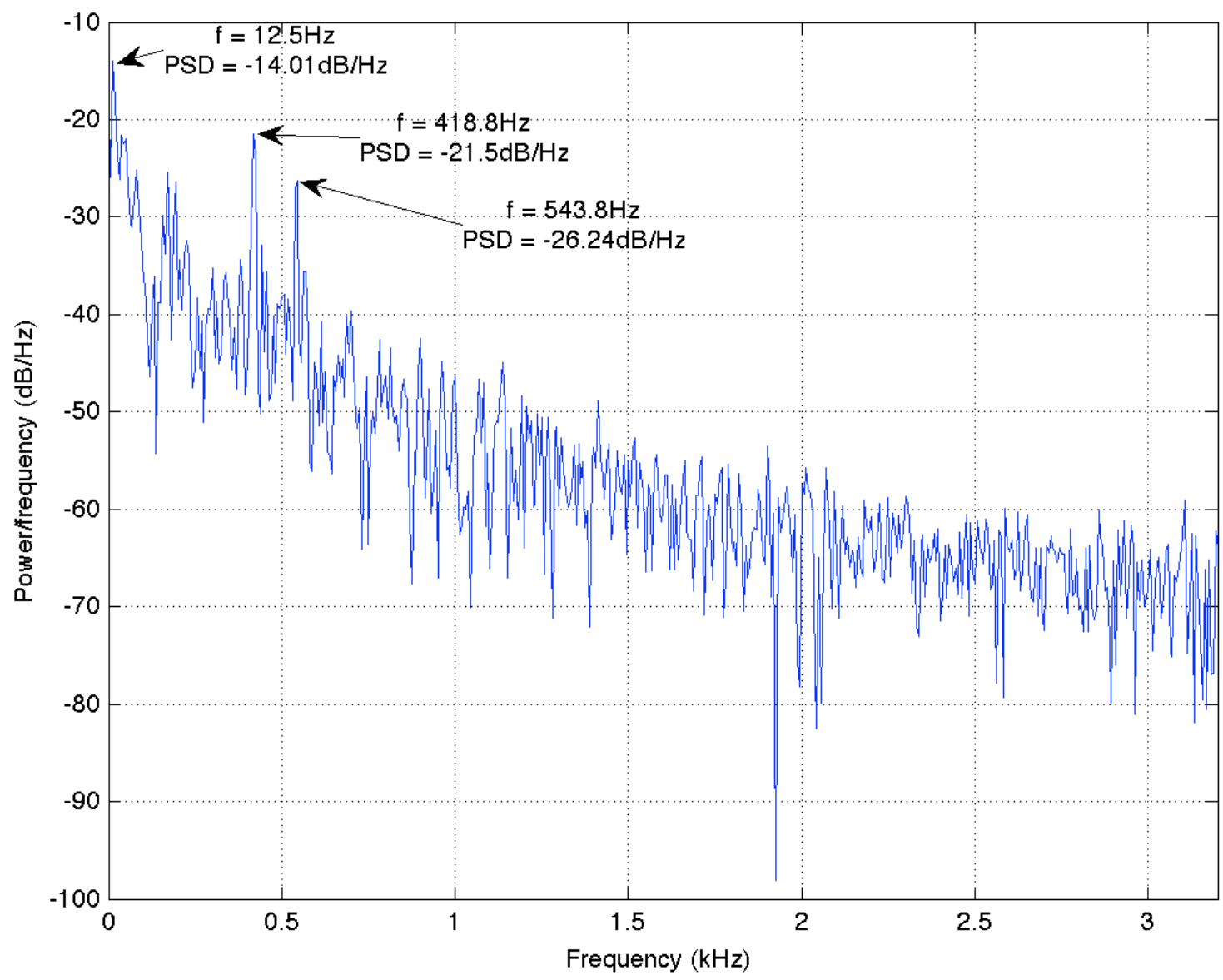

(b) PSD of $Y_{1}(10)$, obtained from the lowest eigenvalue and has multiple sharp peaks Figure 2.4: (cont.) 
and amplitude variations in a component represent the separation between signals from successive trials. Thus, the frequency represents frequent variations between trials and the amplitude represents the amount of variation between the individual trials. Hence, the power spectral density (PSD) (Stoica and Moses, 1997) analysis of these signals will give a better understanding of both the amount of variation and the frequency of variation in order to effectively remove background EEG activity, which is regarded as noise in SSEP signals.

One important observation that can be made was that the lower eigenvalue components had a very sharp power peaks at a single frequency and the higher eigenvalue components had sharp spikes at multiple frequencies as shown in fig. 2.4.

Figure 2.4a has a sharp peak at $18.75 \mathrm{~Hz}$ and the difference between the first two peaks is $27.08 \mathrm{~dB} / \mathrm{Hz}$, indicating that the $18.75 \mathrm{~Hz}$ component contributes 500 times more than the $418.8 \mathrm{~Hz}$ component. In fig. $2.4 \mathrm{~b}$, the difference between the first two peaks is $7.49 \mathrm{~dB} / \mathrm{Hz}$, indicating that the $12.5 \mathrm{~Hz}$ component contributes only 5.6 times more than the $418.8 \mathrm{~Hz}$ component. The cause can be attributed to the statistical variance exhibited by the higher magnitude eigenvalues. The power spectrum was computed using the fast Fourier transform (FFT) method (Frigo and Johnson, 1998).

It can be assumed that since these singular frequency components will contribute solely to their corresponding frequencies they can be eliminated. For reconstruction purposes, the components that have less than two peaks higher than $-30 \mathrm{~dB} / \mathrm{Hz}$ were removed. The $-30 \mathrm{~dB} / \mathrm{Hz}$ threshold was arbitrarily chosen based on the total power contribution of the component. To show the significance of the observation, raw data from the first 10 trials are averaged in time compared with the average of 300 trials from the same subject during the same operation; the low-frequency components 
were estimated and removed and a final signal was obtained as shown in fig. 2.6.

\subsubsection{AMUSE algorithm - Noise components filtering}

These characteristic low frequency components were observed in data obtained from all the test subjects and varied from $6 \mathrm{~Hz}$ to $25 \mathrm{~Hz}$, while the high frequency components were as high as $418 \mathrm{~Hz}$. The frequency and amplitude variations in a component represent the separation between signals from successive trials. Thus, the frequency represents frequent variations between trials and the amplitude represents the amount of variation between the individual trials. Hence, the power spectral density (PSD) analysis of these signals will give a better understanding of both the amount of variation and the frequency of variation in order to effectively remove background EEG activity, which is regarded as noise in SSEP signals.

An important observation that can be made was that the lower eigenvalue components had very sharp power peaks at single frequency and the higher eigenvalue components had sharp spikes at multiple frequencies. The cause can be attributed to the statistical variance exhibited by the higher magnitude eigenvalues. An example of high and low frequency components is presented in fig. 2.5. Notice that the reconstructed signal resembles the desired SSEP waveform in terms of peak latencies more closely upon the removal of the high-power low frequency components.

It can be inferred that since these singular frequency components will contribute solely to their corresponding frequencies they can be eliminated. For reconstruction purposes, the components that have less than two peaks higher than $-30 \mathrm{~dB} / \mathrm{Hz}$ were removed. The $-30 \mathrm{~dB} / \mathrm{Hz}$ threshold was arbitrarily chosen based on the total power contribution of the component. To show the significance of the observation, raw data from the first 10 trials are averaged in time compared with the average of 200 

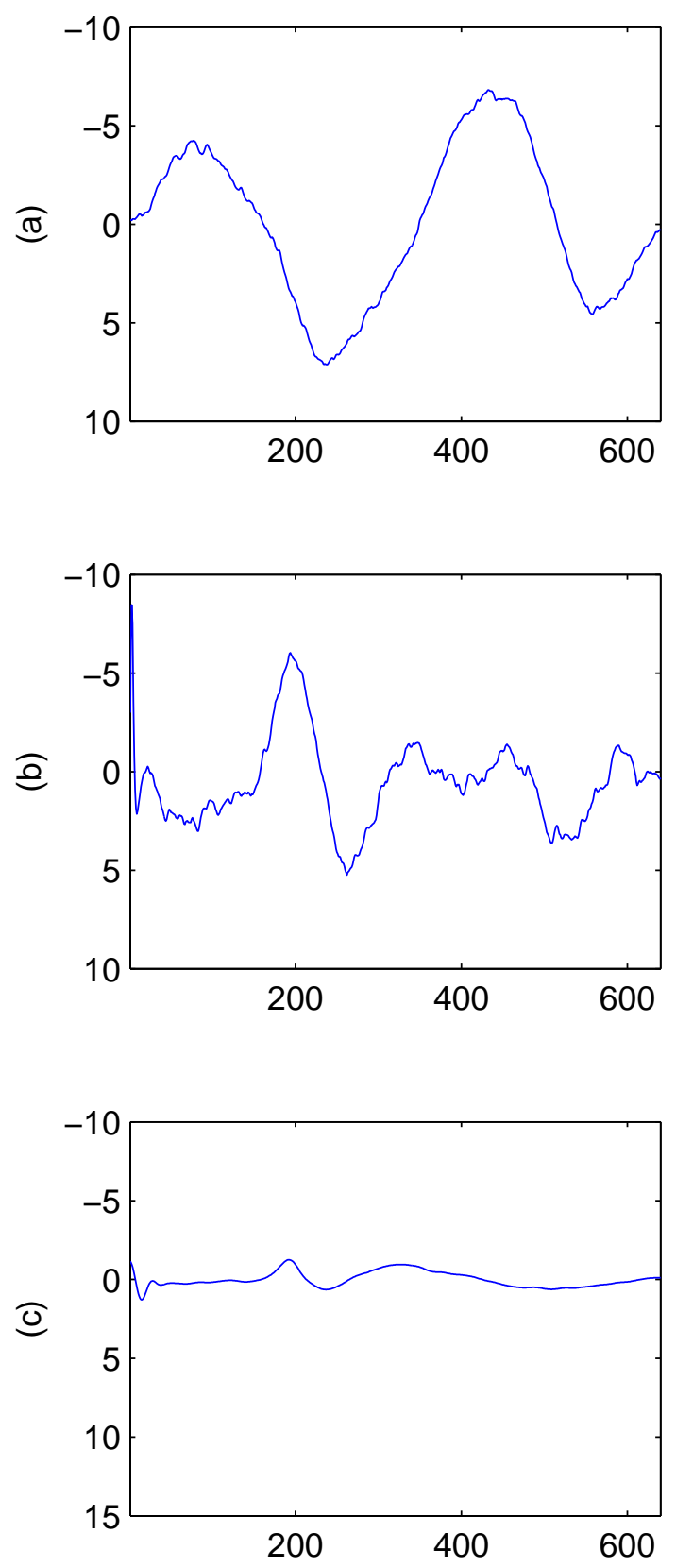

Figure 2.5: Reconstruction of SSEP from high frequency and low frequency components and compared with the known clinical average. (a) SSEP reconstruction after eliminating high-frequency components, (b) SSEP reconstruction after eliminating low-frequency components and (c) the known clinical SSEP waveform. The X-axis represents the time samples and Y-axis represents the amplitude. 
trials from the same subject during the same operation; the low-frequency components were estimated and removed and a final signal was obtained as was shown in fig. 2.6.

It should be indicated that the signal obtained after filtering is still not smooth enough to obtain a valid inference. From the frequency spectra obtained, frequencies higher than $250 \mathrm{~Hz}$ were randomly distributed and hence need to be filtered out from the signal. The average signal obtained was filtered using an eighth order Butterworth LPF with a cut-off frequency of $250 \mathrm{~Hz}$. The signal was then tested for the maxima and minima using the first order and second order differentials. The peaks in the Walsh-transformed signal are then used to automate the identification of the evoked response. A difference operator can be used but they have a serious drawback of being highly susceptible to noise, and thus a smoothing operator need to be included to improve the differentiator's signal to noise ratio (SNR). The Walsh differentiation method (Adjouadi et al., 2004, 2005; Weide et al., 1978) was utilized to overcome the problem, as described in the following section.

\subsubsection{Walsh transform - Automated peak detection}

The second order Walsh operator of length four $\left(W_{N}^{r} \equiv W_{4}^{2}\right)$, equivalent to a fourpoint second- order differentiation operator, was convolved with the average signal to obtain a Walsh-transformed signal whose peak location is found to determine the peak locations of the SSEP (at least for $70 \%$ of the cases where noise was not preponderant). The magnitude of each of the points as a function of time of this Walsh-transformed signal was defined by performing the following convolution:

$$
\mathbf{W}=\frac{1}{4}\left(w_{4}^{2} * \mathbf{X}_{\text {final }}\right)
$$


where, $w_{4}^{2}=\left[\begin{array}{llll}1 & -1 & -1 & 1\end{array}\right]$ is the Walsh kernel, which is functionally similar to the second-order derivative and the center point difference $\left[\begin{array}{ccc}1 & -2 & 1\end{array}\right]$, and where the symbol '*' represents the convolution operation. The peaks of $\mathbf{W}$ are obtained to localize the two peaks of the SSEP response corresponding to P37 and N45 for the tibial nerve. The Walsh maximum always corresponds to either P37 or N45 that is verified from the sign of the amplitude of the signal at the detected time instance. Once the maximum peak is obtained automatically, the next maximum point with opposite polarity defines the second peak.

Figure 2.7 shows the process as it applies for subject 2 and as can be seen the method effectively filters the noise and highlights the evoked response with just 10 trials. The figure compares the time occurrences of Walsh transform peaks detected on the given average signal in fig. 2.7(a) with that from the signal obtained from 10 trials using the proposed algorithm in fig. 2.7(b). The algorithm was successfully implemented on 20 subjects ensuring the repeatability of the algorithm.

A similar Walsh transformation when squared can be easily compared with the second order derivative. Figure 2.8 shows such an implementation wherein the Walsh transform is able to detect the SSEP peaks even though the morphology of the signal is incomprehensible.

\subsection{Implementation Results}

The details on the trials and SSEP locations of the 20 subjects included in the study are summarized in tables 2.1a to 2.1d (Vedala et al., 2012b,a). For each of the first 16 subjects, table 2.1a provides the SSEP response locations for the two bipolar recording channels obtained clinically and are contrasted to those locations 


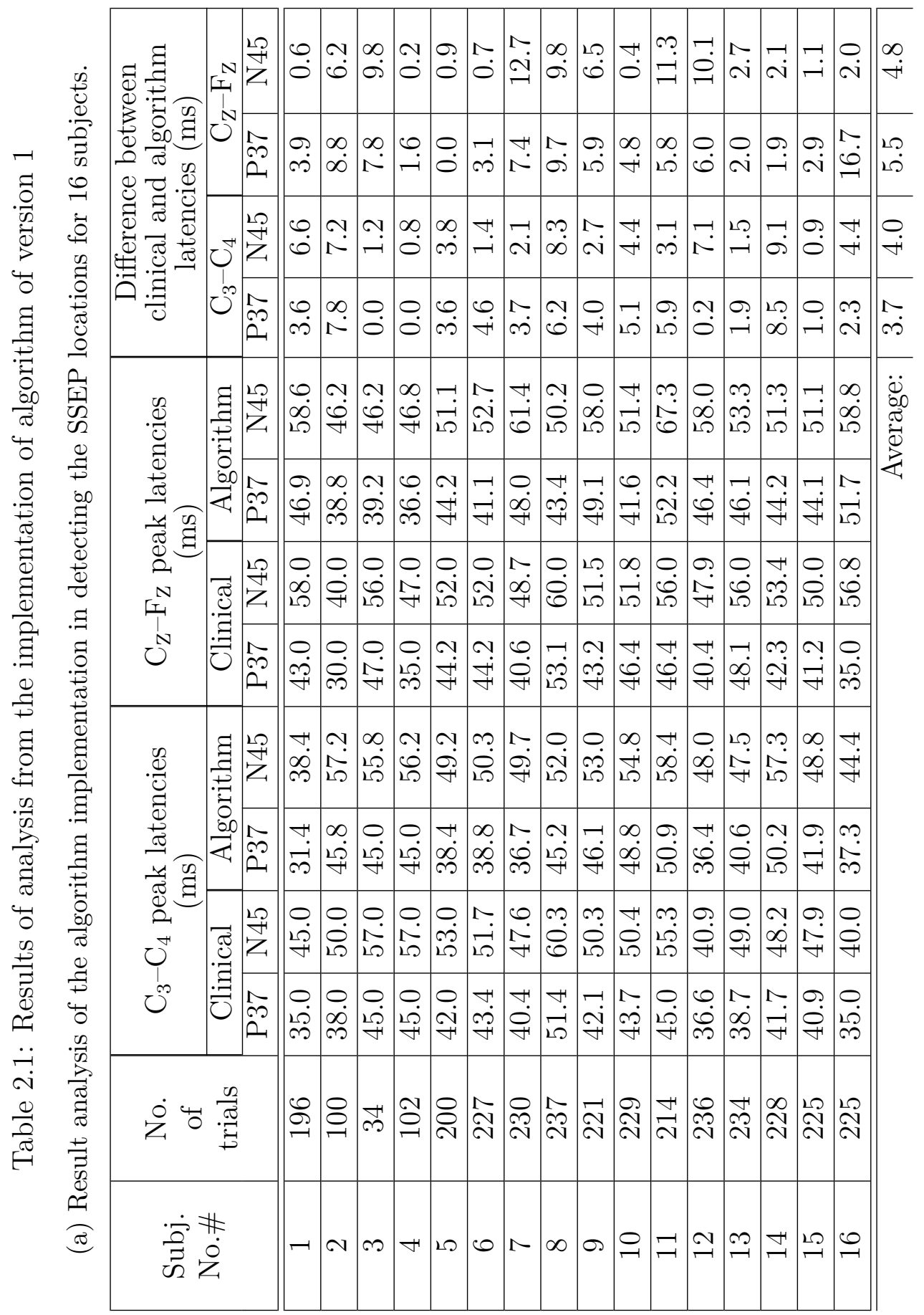




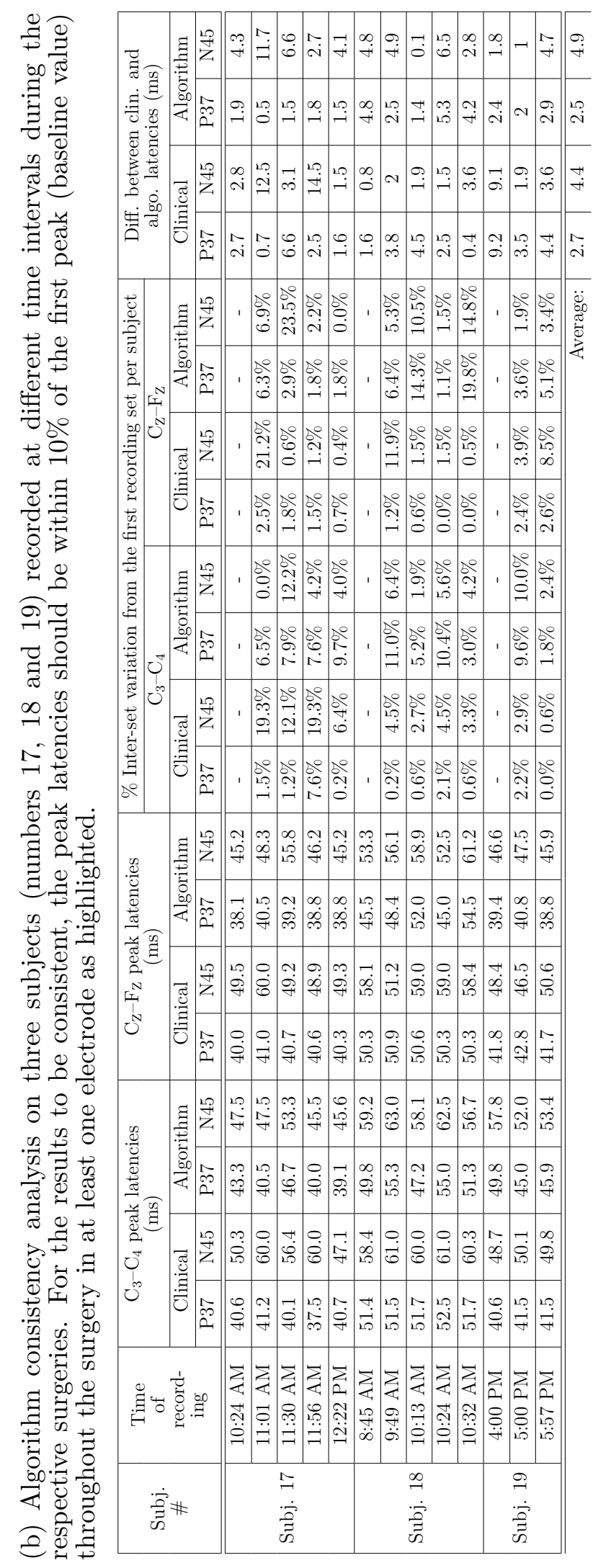




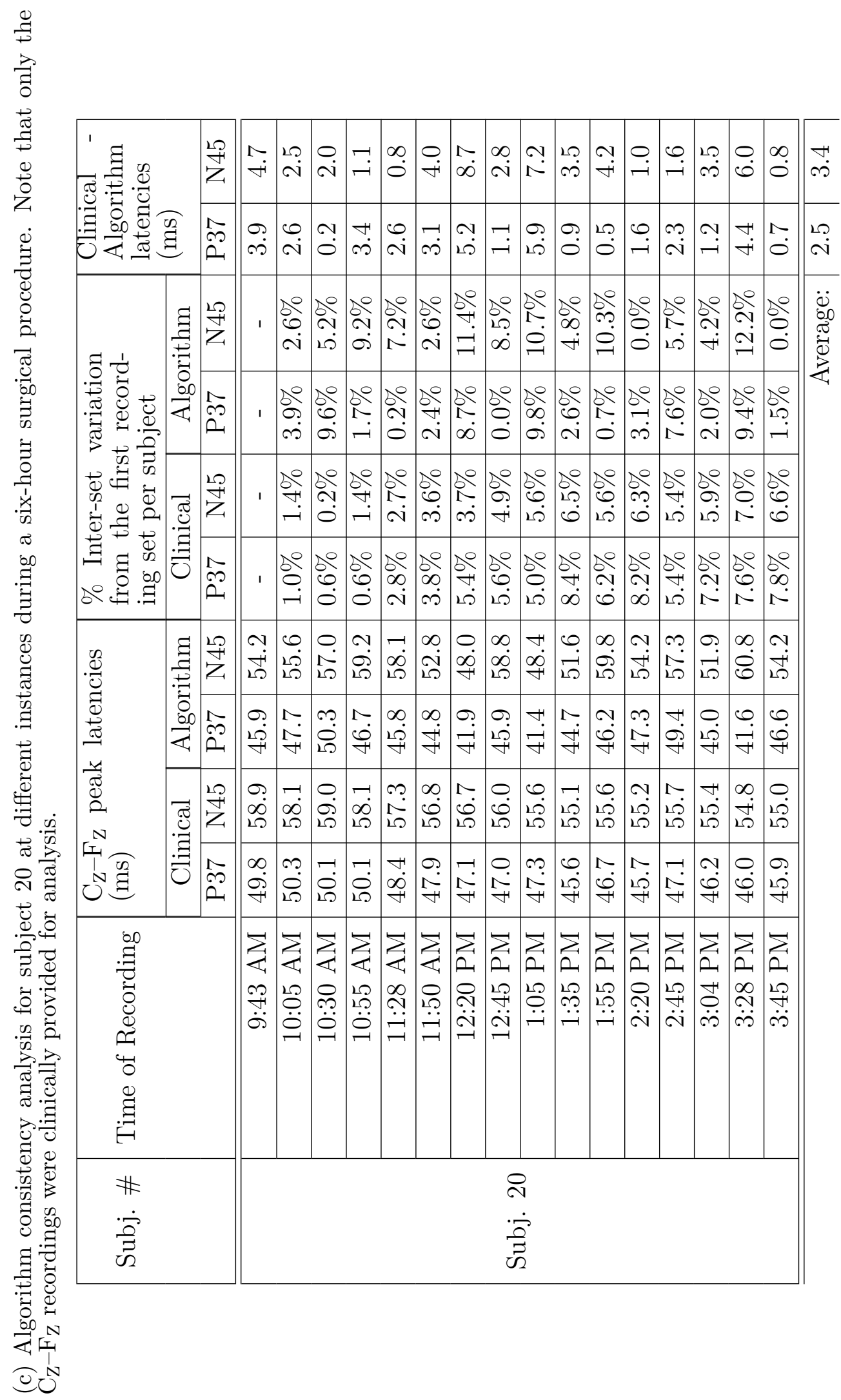


(d) Amplitude consistency for subjects 17 thru 20 corresponding to the peak latencies shown in tables 2.1b and 2.1c. For Subjects 17, 19 and 20, the amplitudes are from $\mathrm{C}_{\mathrm{Z}}-\mathrm{F}_{\mathrm{Z}}$ channel and for subject 18 from the $\mathrm{C}_{3}-\mathrm{C}_{4}$ channel.

\begin{tabular}{|c|c|c|c|}
\hline Subj. \# & $\begin{array}{l}\text { Time of } \\
\text { recording }\end{array}$ & P-P Ampl. $(\mu \mathrm{V})$ & P-P Ampl. Error (\%) \\
\hline \multirow{5}{*}{ Subj. 17} & $10: 24 \mathrm{AM}$ & 0.66 & - \\
\hline & 11:01 AM & 0.57 & $13 \%$ \\
\hline & 11:30 AM & 0.65 & $2 \%$ \\
\hline & $11: 56 \mathrm{AM}$ & 0.93 & $41 \%$ \\
\hline & 12:22 PM & 0.61 & $9 \%$ \\
\hline \multirow{5}{*}{ Subj. 18} & $8: 45 \mathrm{AM}$ & 0.53 & - \\
\hline & 9:49 AM & 0.28 & $47 \%$ \\
\hline & 10:13 AM & 0.46 & $12 \%$ \\
\hline & 10:24 AM & 0.50 & $5 \%$ \\
\hline & 10:32 AM & 0.63 & $19 \%$ \\
\hline \multirow{3}{*}{ Subj. 19} & 4:00 PM & 0.51 & - \\
\hline & 5:00 PM & 0.73 & $43 \%$ \\
\hline & $5: 57 \mathrm{PM}$ & 0.52 & $2 \%$ \\
\hline \multirow{16}{*}{ Subj. 20} & 9:43 AM & 0.91 & - \\
\hline & 10:05 AM & 0.86 & $5 \%$ \\
\hline & 10:30 AM & 0.70 & $23 \%$ \\
\hline & $10: 55 \mathrm{AM}$ & 0.62 & $32 \%$ \\
\hline & $11: 28 \mathrm{AM}$ & 0.48 & $47 \%$ \\
\hline & $11: 50 \mathrm{AM}$ & 0.65 & $28 \%$ \\
\hline & $12: 20 \mathrm{PM}$ & 0.85 & $6 \%$ \\
\hline & 12:45 PM & 0.82 & $10 \%$ \\
\hline & 1:05 PM & 1.00 & $10 \%$ \\
\hline & $1: 35 \mathrm{PM}$ & 0.74 & $18 \%$ \\
\hline & 1:55 PM & 0.51 & $44 \%$ \\
\hline & $2: 20 \mathrm{PM}$ & 0.66 & $27 \%$ \\
\hline & 2:45 PM & 0.70 & $23 \%$ \\
\hline & 3:04 PM & 0.87 & $4 \%$ \\
\hline & $3: 28 \mathrm{PM}$ & 0.92 & $2 \%$ \\
\hline & $3: 45 \mathrm{PM}$ & 0.74 & $18 \%$ \\
\hline
\end{tabular}


determined by the proposed algorithm. It includes the error (difference) in milliseconds (ms) of the estimated SSEP location with respect to the corresponding actual location as provided by the clinicians. For 14 of these 16 subjects, the location determined by the proposed algorithm relied solely on the first ten trials or recorded signals. For the remaining two subjects, it was noted that two of the first ten trials were corrupted and were replaced by the successively recorded signals to constitute the required 10 trials, for consistency purposes.

With the initial findings in using these 16 subjects, four more subjects were added to the study to assess consistency in detecting the baseline peak latencies. To prove the plausibility of an SSEP monitoring system, the system should show consistency at different times in a single surgical procedure. Four such cases were then obtained and analyzed for at least one consistent peak throughout the procedure. The highlighted entries show the consistency of the peaks i.e., within $\pm 10 \%$ of the first peak latency (baseline value) as detected by the algorithm using ten trials. Tables 2.1b to $2.1 \mathrm{~d}$ show recordings at different times through these added four surgeries and a comparison of peaks is provided between the estimated peak latencies using the algorithm and those provided clinically.

It can be seen from tables $2.1 \mathrm{a}$ to $2.1 \mathrm{~d}$ and figs. 2.7 and 2.9 that the algorithm output from ten trials using the proposed algorithm closely mimics (within a $10 \%$ time latency deviation and within 50\% peak-to-peak amplitude deviation) the average signal obtained clinically using a multitude of trials and the response could be clearly visualized. This algorithm is simple enough to be implemented in the recording device itself.

Present day recording systems, such as CASCADE® Intra-operative Monitoring rely 
on amplitude threshold or area under the curve schemes for the SSEP peak detection. A well-defined criterion needs to be applied to appropriately remove the noisy trial recordings and a much better response can be obtained while still contending with a limited number of trials. Another observation is that the individual trials show a typical frequency pattern wherein the average power density in the frequency range of $0 \mathrm{~Hz}$ to $50 \mathrm{~Hz}$ is at least $10 \mathrm{~dB} / \mathrm{Hz}$ greater than that in the frequency range of $100 \mathrm{~Hz}$ to $200 \mathrm{~Hz}$. This information can be utilized to implement automated noisy signal elimination and enhance the performance of the system. The algorithm implemented for all the subjects achieved very promising SSEP detection results with an accurate detection in at least one bipolar recording channel.

With all these results, a retrospective on the merits of the proposed algorithm helps us to

- identify and get rid of the corrupted SSEP signal components,

- determine the time (latency) variations in different SSEP trials and

- justify our assumption of independent SSEP trial signals.

The drawback of the algorithm was that the resulting SSEP waveform might not be true to its morphology even though the Walsh transform is able to detect the peaks. This issue was addressed in version 2 of the algorithm. 


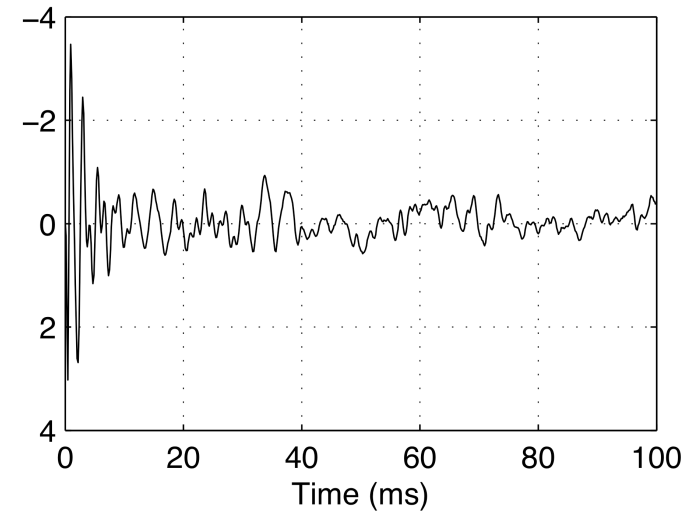

(a) Average of 10 raw trials

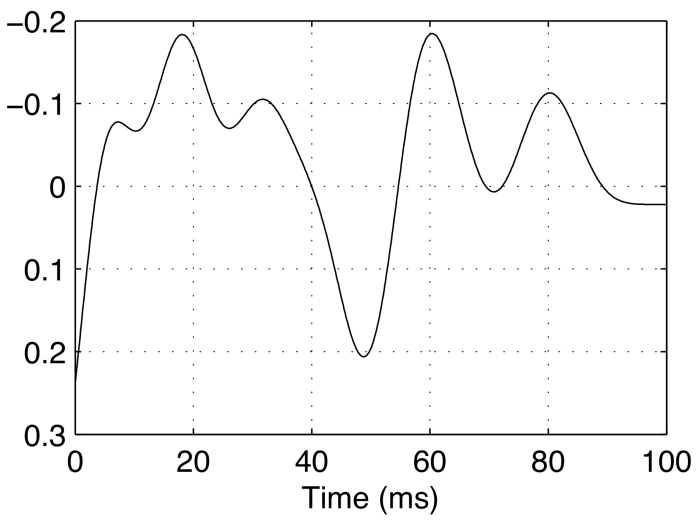

(c) Filtered signal from 10 trials

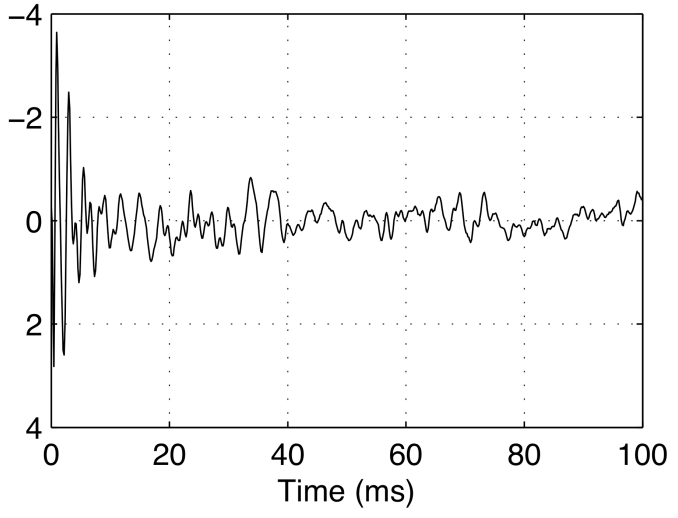

(b) Low frequency components

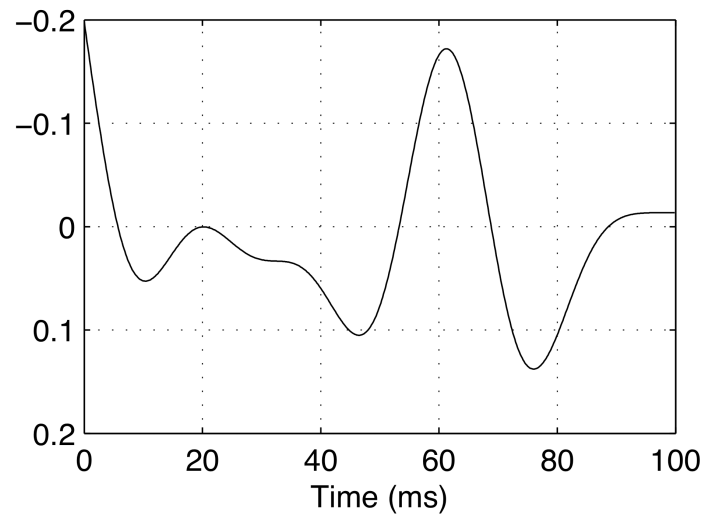

(d) Average signal from 200 trials

Figure 2.6: Significance of removing the low-frequency components: (a) Average of raw signals from 10 trials, (b) noise components estimated from the 10 trials in fig. 2.3, (c) average of raw signals from 200 trials and (d) signal obtained after filtering signal in (a) showing the possible location of the SSEP response. The X-axis represents the time in seconds. 

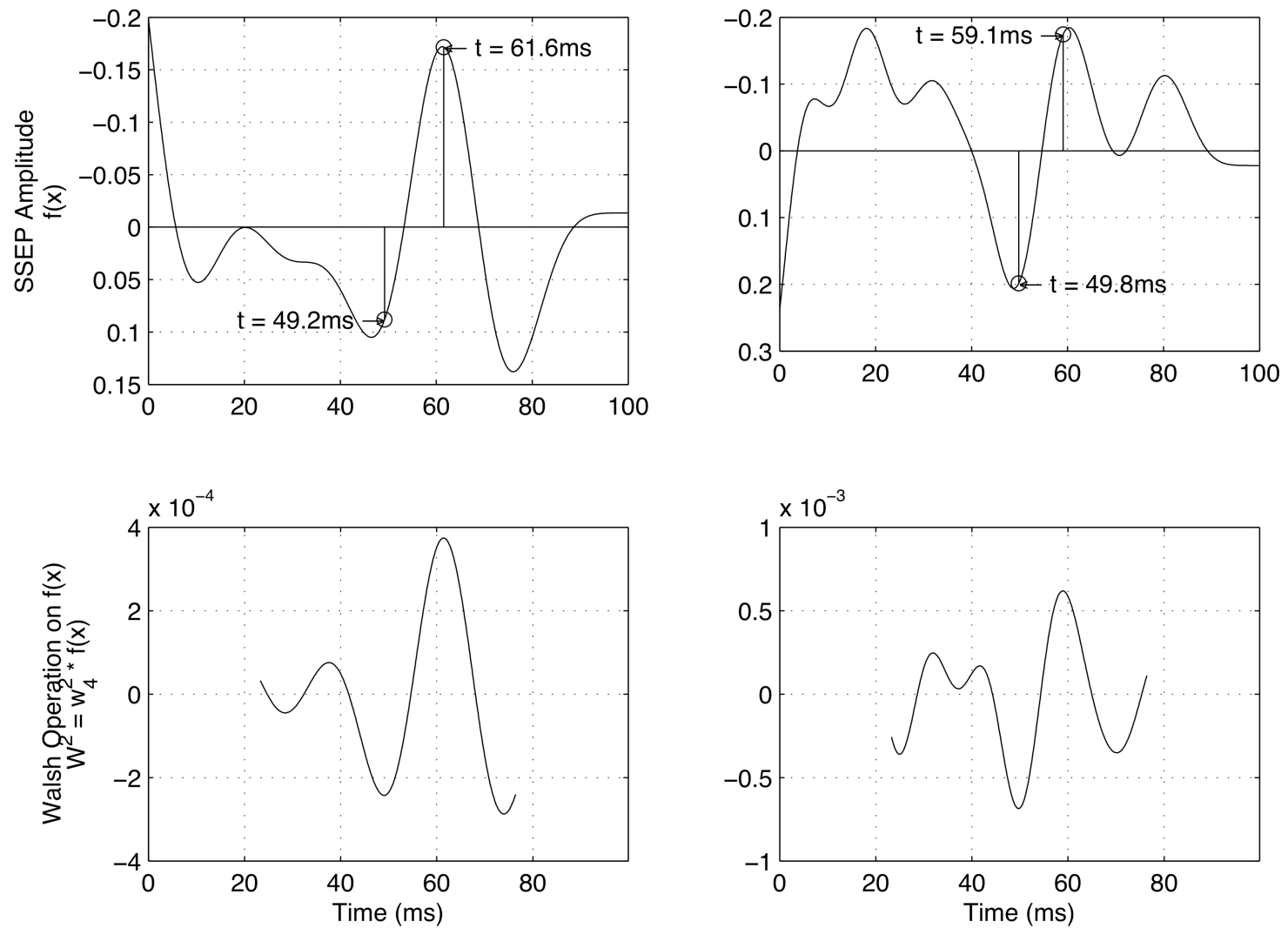

(a) Given signal using 200 trials and its corresponding Walsh transform

(b) Algorithm signal using 10 trials and its corresponding Walsh transform

Figure 2.7: Application of Walsh transform to identify the position of the SSEP: (a) Left column displays the signal obtained using conventional averaging. The plot on the top left shows the given average signal obtained from 200 trials and the possible location of evoked potential response using the Walsh operation shown below it. (b) Right column displays the signal obtained using the proposed algorithm and the detected location of evoked potential response obtained using the Walsh operation shown below it. 

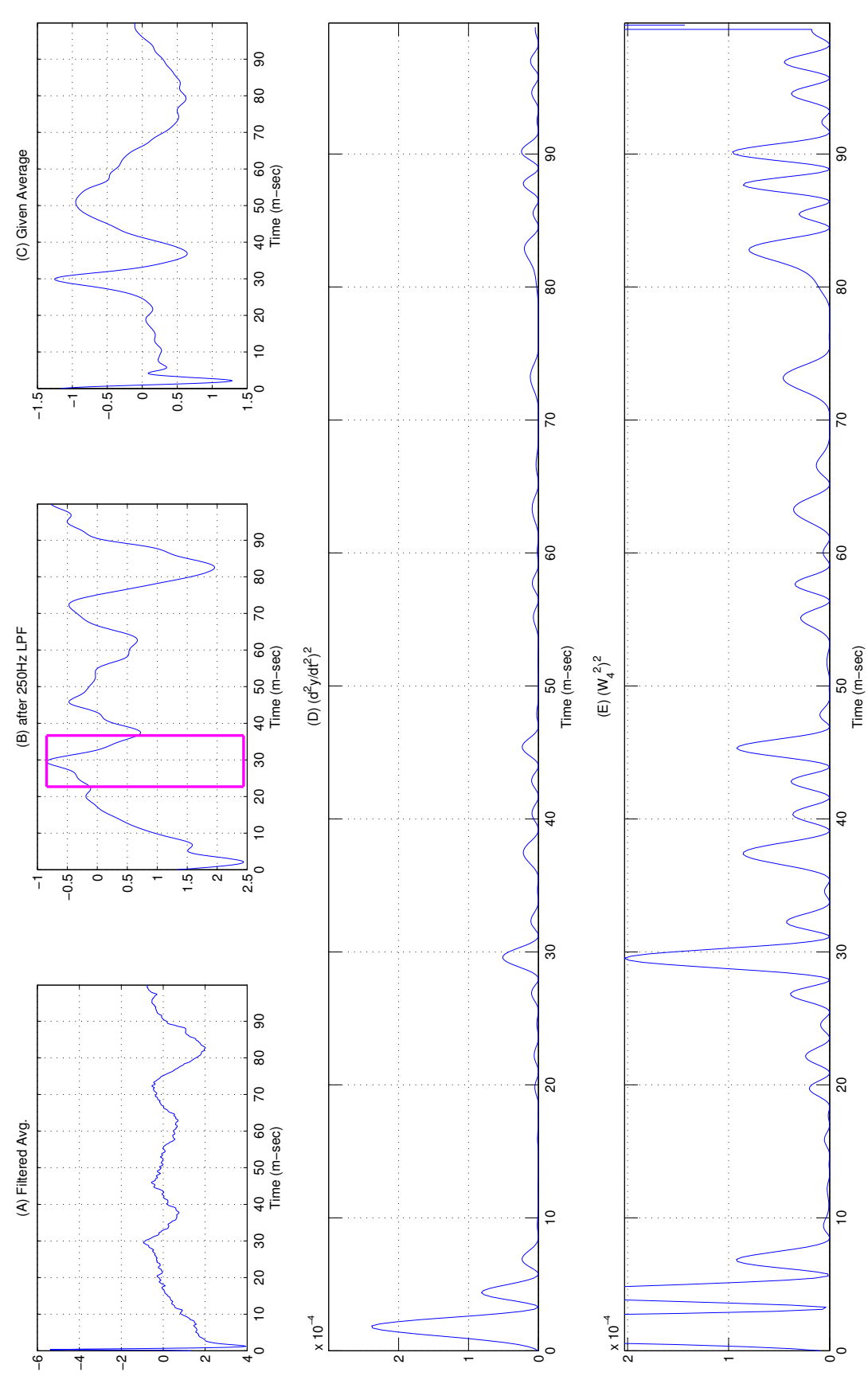

耳访

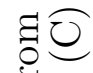

$\exists \frac{\dot{n}}{\sigma}$

.7. 50

융

สํํㅇ

की

00

.

40

\&

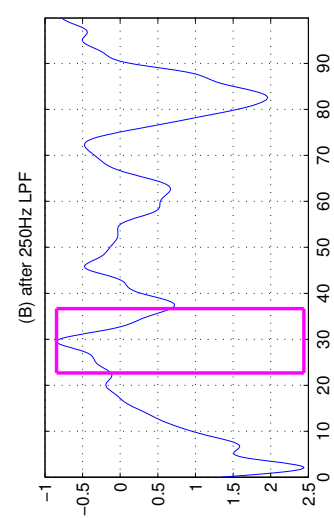

E 50 s

स.

$\dot{0} \overrightarrow{0}$

卷

으

घ

胥:

0.0

颔牙

导它

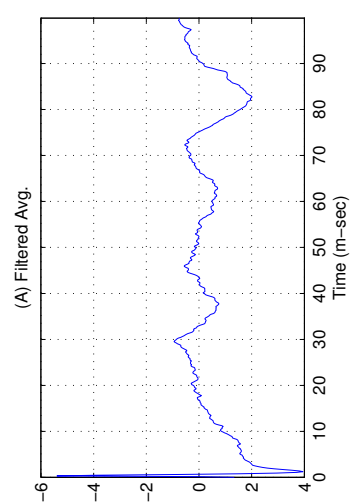

엉

ช。

पै

न्व

ن.

苋团

ช.ำ

䲶通过

预

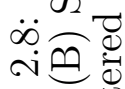

○

我 


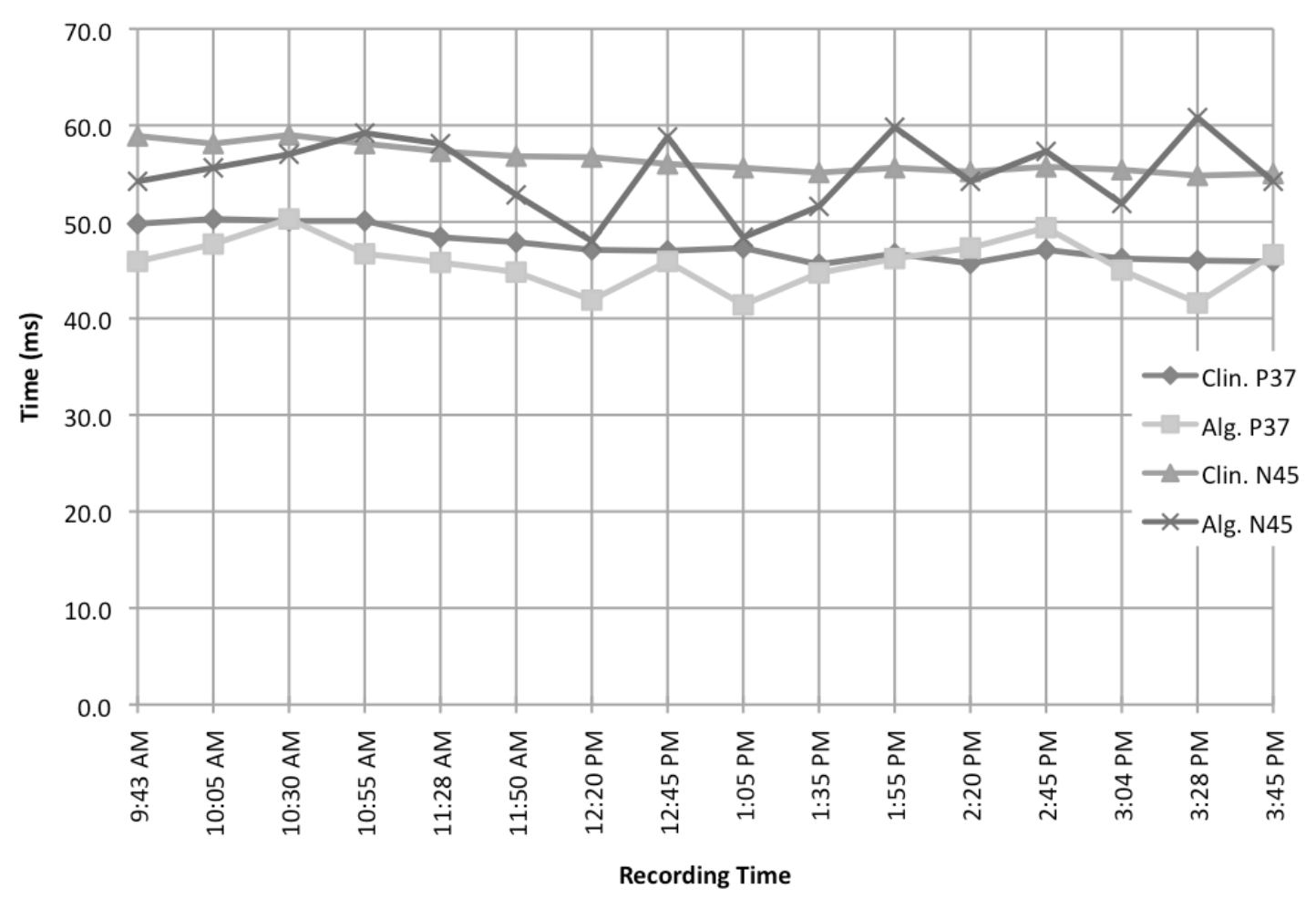

Figure 2.9: Consistency in detecting P37 and N45 peak latencies from the $\mathrm{C}_{\mathrm{Z}}-\mathrm{F}_{\mathrm{Z}}$ recording of subject 20 with the peak latencies (algorithm vs. clinical) as given in table $2.1 \mathrm{~d}$. 


\section{CHAPTER 3}

\section{ALGORITHM VERSION 2 - TEMPORAL FILTERING}

\subsection{Background}

Version 1 of the algorithm was proven to be successful in identifying the SSEP peaks using mathematical tools while not limiting to signal morphology. However, if the SSEP was indeed extracted, the SSEP waveform should be clearly identified by the peaks and the signal morphology. Keeping this in mind, it was understood that the morphology of the signal, was present in the temporal domain.

\subsection{Gaussian Template}

The first temporal filtering approach developed by our group developed was based on Gaussian template (Goryawala et al., 2012, 2011). The algorithm steps are summarized as follows:

(a) Noise removal: Any trial with a standard deviation greater than the mean of standard deviations of the set for base-line is considered a noisy trial and is rejected.

(b) Three step filtering: $60 \mathrm{~Hz}$ noise, $300 \mathrm{~Hz}$ LPF and 25-point moving average filter for signal smoothing.

(c) Averaging: The signals are now averaged similar to clinical approach in time to obtain the SSEP and position and amplitude are obtained.

(d) Gaussian template: A patient specific template was generated from the base-line signal and convolved with the average signal obtained from step C. 
This algorithm used the conventional averaging method to obtain the baseline signal for the specific subject. The algorithm steps were then applied on every 20 consecutive trials to extract the SSEP as shown in fig. 3.1. This baseline signal was then used in the subsequent steps to obtain the trial rejection criterion and to generate the Gaussian template using the equation:

$$
g(x)=a \cdot \exp \left[\frac{(x-b)^{2}}{2 c^{2}}\right]
$$

where $a$ is scaling factor for normalization, $b$ is the mid-point between the two peaks and $c$ is chosen such that the full width at half maximum (FWHM) of the template is twice the time difference between the two peaks. The implementation results from the algorithm are not presented in lieu of the merit of the following Chebyshev filtering method but can be obtained from our research group (Goryawala et al., 2012, 2011).

\subsection{Chebyshev Filtering}

Upon establishing the fact that the morphology of SSEP was associated with temporal information, our group then improvised and developed a Chebyshev based filter. The Gaussian template approach required a priori-information of the peak latencies for a patient that was obtained by conventional averaging for the first 200 trials. Moreover, like conventional averaging, a linear digital filter with linear phase response should be utilized. Addressing these issues, our team developed a finite impulse response (FIR) filter was developed with the understanding that typically, the tibial SSEP waveform is centered at $41 \mathrm{~ms}$ (mean of $37 \mathrm{~ms}$ and $45 \mathrm{~ms}$ ).

In fact if a filter modifies the appearance of the SSEP signal in time domain the po- 
sition of positive and negative peaks and their amplitude may change dramatically. Linear phase filters are known for their performance in preserving the signal shape in time domain. In fact, most of the commercial SSEP monitoring devices implement some kind of moving average, which is linear phase by definition. Here, Fast Fourier Transform (FFT) based filtering was used because of the sliced nature of the data for SSEP trial. FFT application requires some form of windowing in time domain to limit the power leakage between frequency components. On the other hand, normal SSEP signal is centered in the first half of a sweep, which would be typically around $41 \mathrm{~ms}$ in a $100 \mathrm{~ms}$ sweep. In order to minimize SNR loss due to windowing the signal was zero padded in the beginning (or end depending on normal SSEP of the subject) to move the SSEP to the middle of the window function where the tapering effect is minimum. A Chebyshev window was applied to the time domain zero padded signal that minimized the Chebyshev norm of the side lobes while increasing the main lobe width on each FFT component that are going to be calculated. FFT was then applied to the signal transferring it to the frequency domain. A rectangular window in frequency domain annihilated the undesired components, which were set to be below $50 \mathrm{~Hz}$ and over $120 \mathrm{~Hz}$. Setting the corresponding component to zero also rejected the $60 \mathrm{~Hz}$ noise due to power equipment. Inverse Fast Fourier transform (IFFT) was then applied on the remaining components resulting in a signal with unwanted components attenuated and the general morphology of the SSEP remaining intact. The zero padded time domain samples were dropped maintaining the overall length of the signal in time domain.

Thus, this method achieves all the steps of Gaussian template type filtering in only three steps. An implementation example of Chebyshev filtering is showing in fig. 3.2a. It can be observed that the Chebyshev filtering has already extracted 
the morphology of the SSEP signal from one trial. However, the morphology may not always be true to the characteristics due to the unpredictable nature of the background brain activity as shown in fig. $3.2 \mathrm{~b}$.

To analyze the effectiveness of the algorithm, we check the SNR for the algorithm with that of the clinical approach. The formula for calculating the SNR is as follows:

$$
\mathrm{SNR}=20 \times \log _{10}\left(\frac{\mathrm{SSEP} \text { signal amplitude }}{\text { noise amplitude }}\right)
$$

The signal is obtained by taking the ensemble average of all the available trials and the noise signal is obtained by alternate addition and subtraction of all the available trials. This method allows us to obtain the SSEP signal and the noise without the SSEP signal at both the input and output of the algorithm (MacDonald et al., 2005). Figure 3.3 shows a typical SNR comparison wherein the proposed Chebyshev filtering provides with an SNR of $6.58 \mathrm{~dB}$ using just 12 trials as compared with clinical averaging with takes as many as 114 trials to achieve the same SNR. 


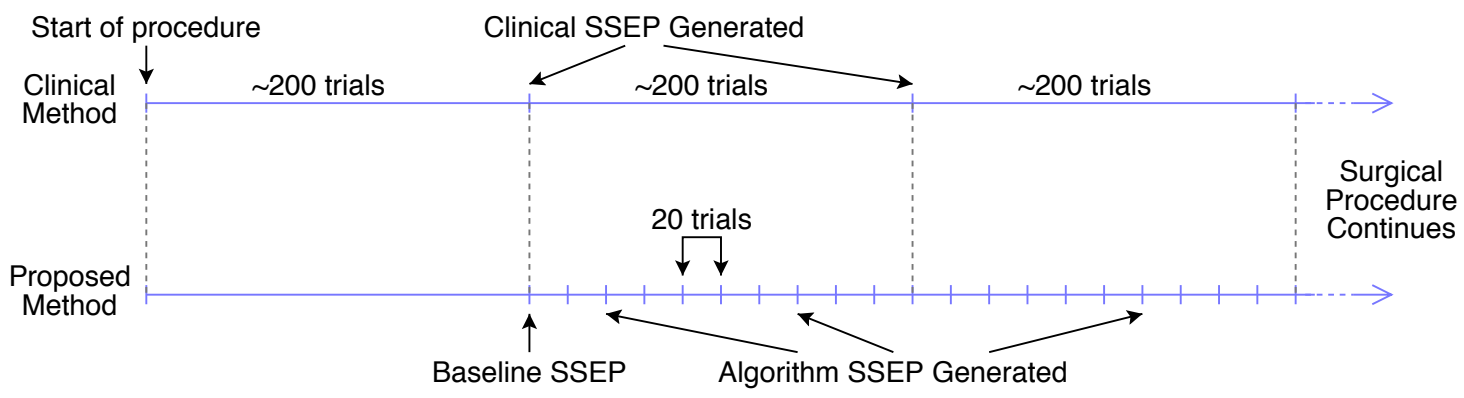

Figure 3.1: Outline of the SSEP recording timeline as compared with the clinical monitoring. By the time one clinical SSEP is obtained, the algorithm version 2 obtained 10 SSEP signals.

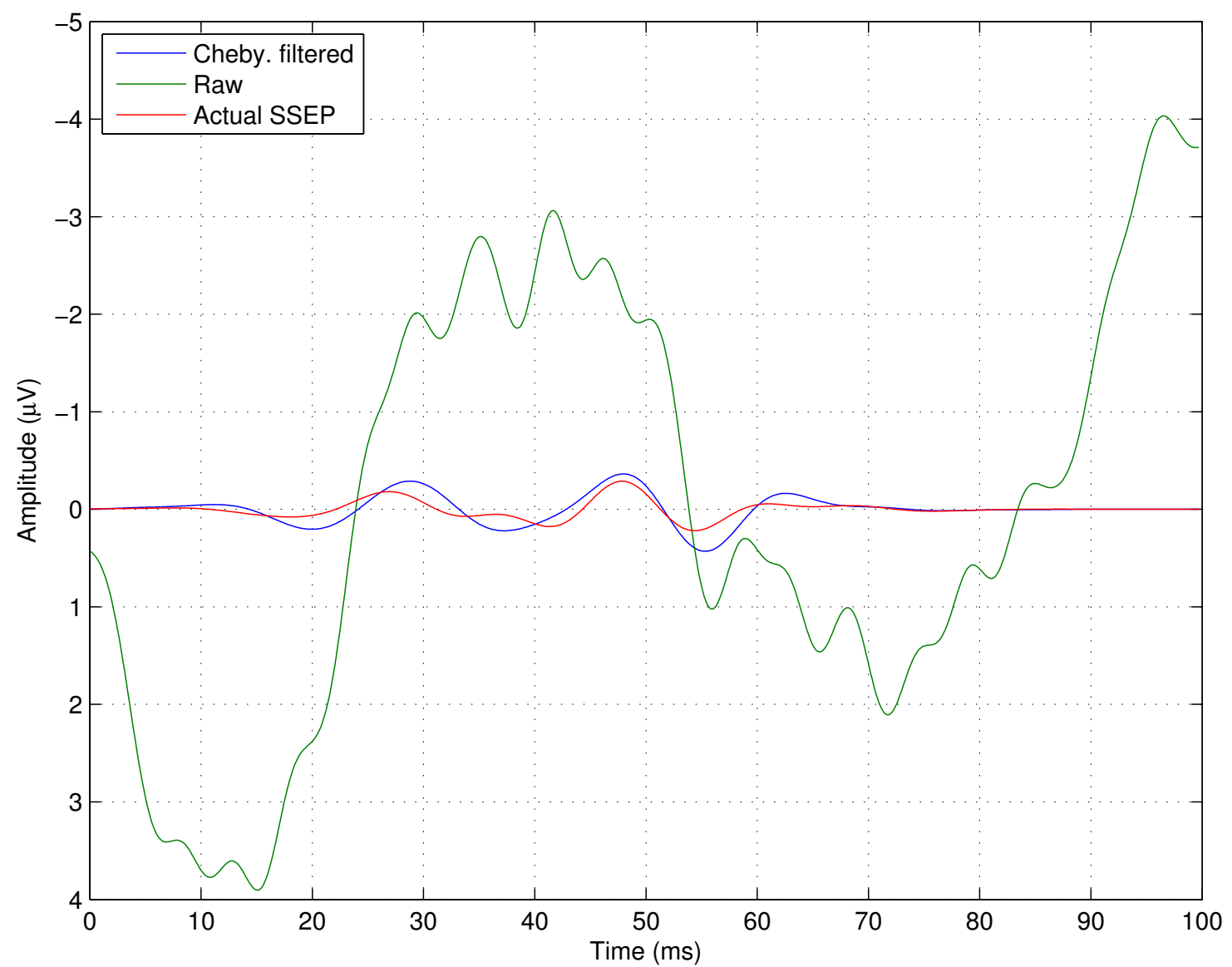

(a) Morphology clearly identifiable. 


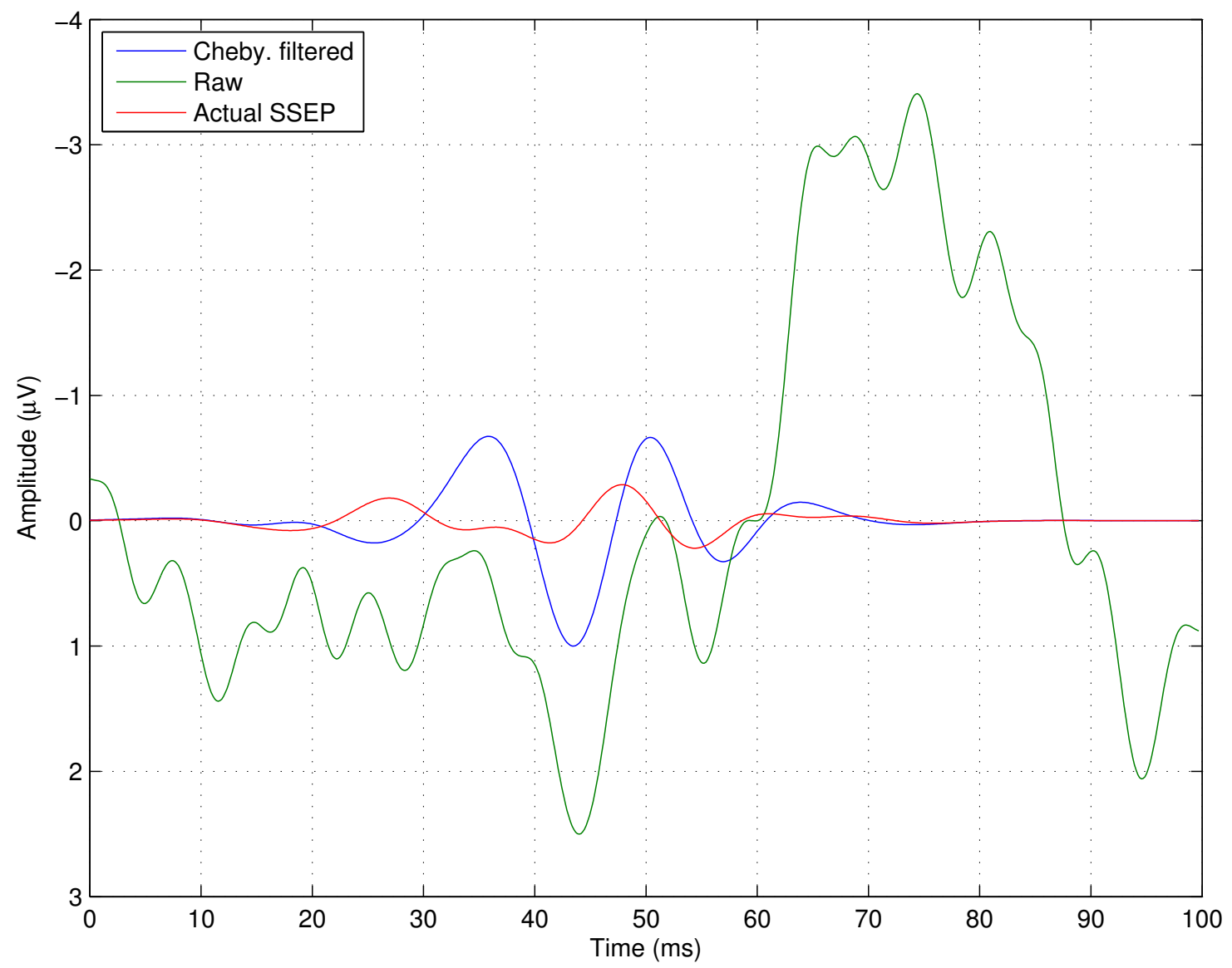

(b) Morphology not so clearly identifiable.

Figure 3.2: Comparison of signals before and after applying Chebyshev filtering with the actual average signal. 


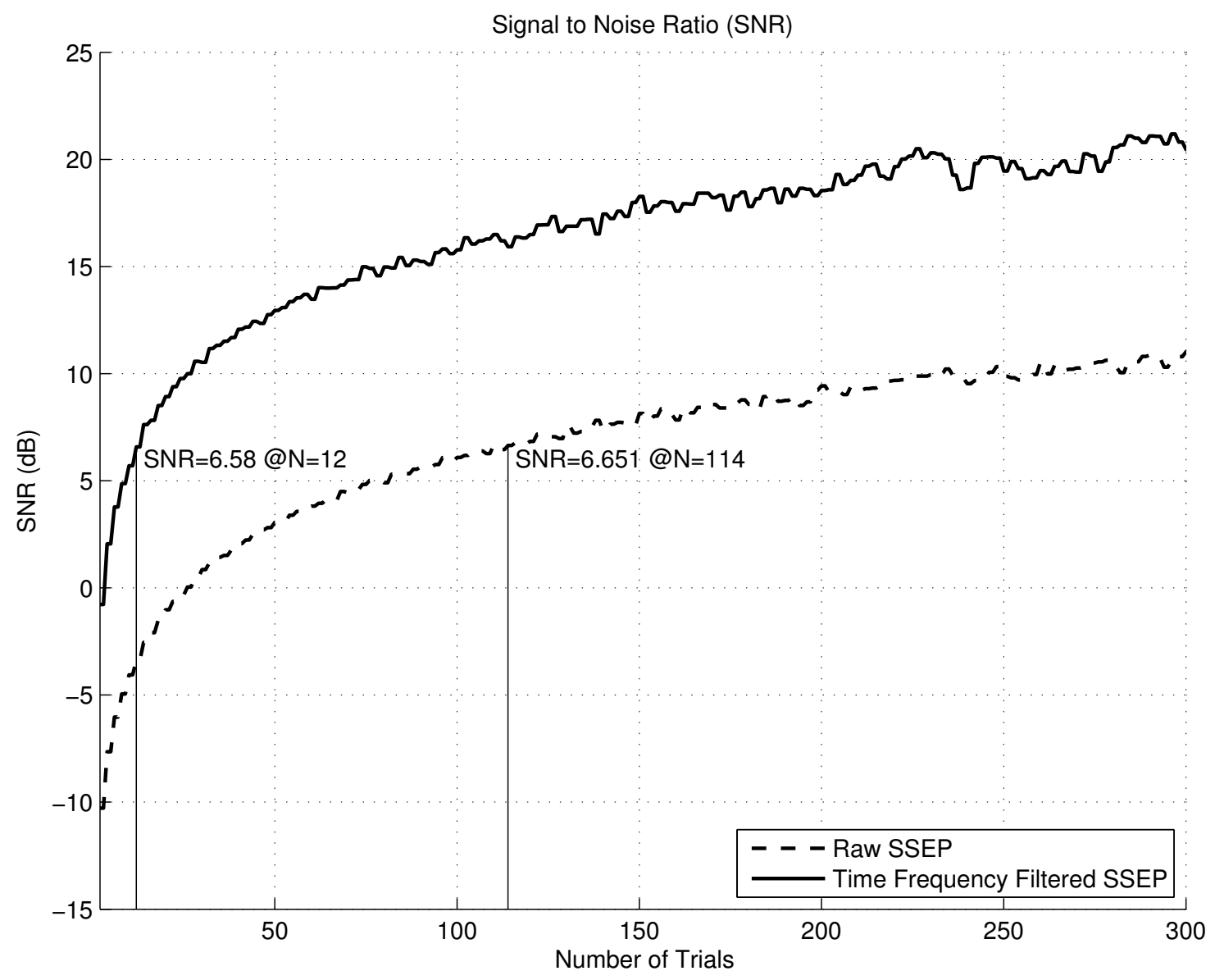

Figure 3.3: The signal to noise ratio (SNR) comparison for raw signals and after. SNR values are provided for the two cases when 12 trials are used and for $200 \mathrm{raw}$ trials. 


\section{CHAPTER 4 \\ ALGORITHM VERSION 3 - COMBINATION OF EIGEN \& TEMPORAL FILTERING}

Version 1 of the algorithm involved direct application of the eigen based filtering on the recorded data and followed by frequency based filtering. In version 2 , a frequency based Gaussian template was used to achieve the same goal. The combination of the two opens up a wider angle of view to understand better the physiology of SSEP. A flowchart which summarizes the key steps taken is shown in fig. 4.1.

\subsection{Merits of Pre-filtering}

Linear phase filters are known for their performance in preserving the signal shape in the time domain. In fact, most of the commercial SSEP monitoring devices implement some kind of moving average, which is linear phase by definition. Here, FFT based filtering was used as explained in the previous chapter.

A Chebyshev window is applied in this case to the time domain zero padded signal that minimizes the Chebyshev norm of the side lobes while increasing the main lobe width on each FFT component that are going to be calculated.

\subsection{Implementation}

\subsubsection{Data Acquisition}

The data acquisition process in this implementation involved creating a matrix of 12 successive unrejected trials for each bipolar recording channel. The same rejection 


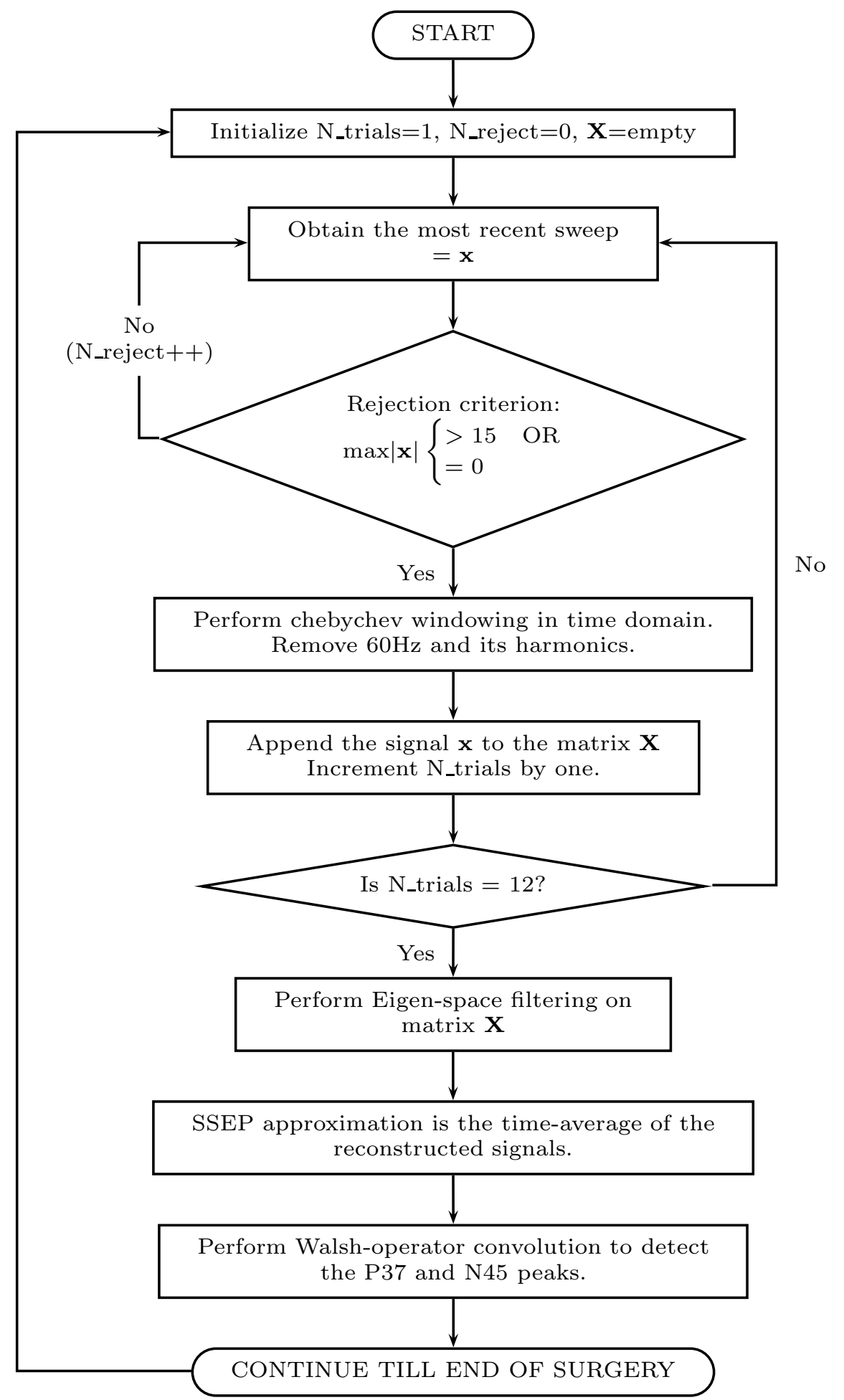

Figure 4.1: Flowchart for the complete algorithm version 3. 

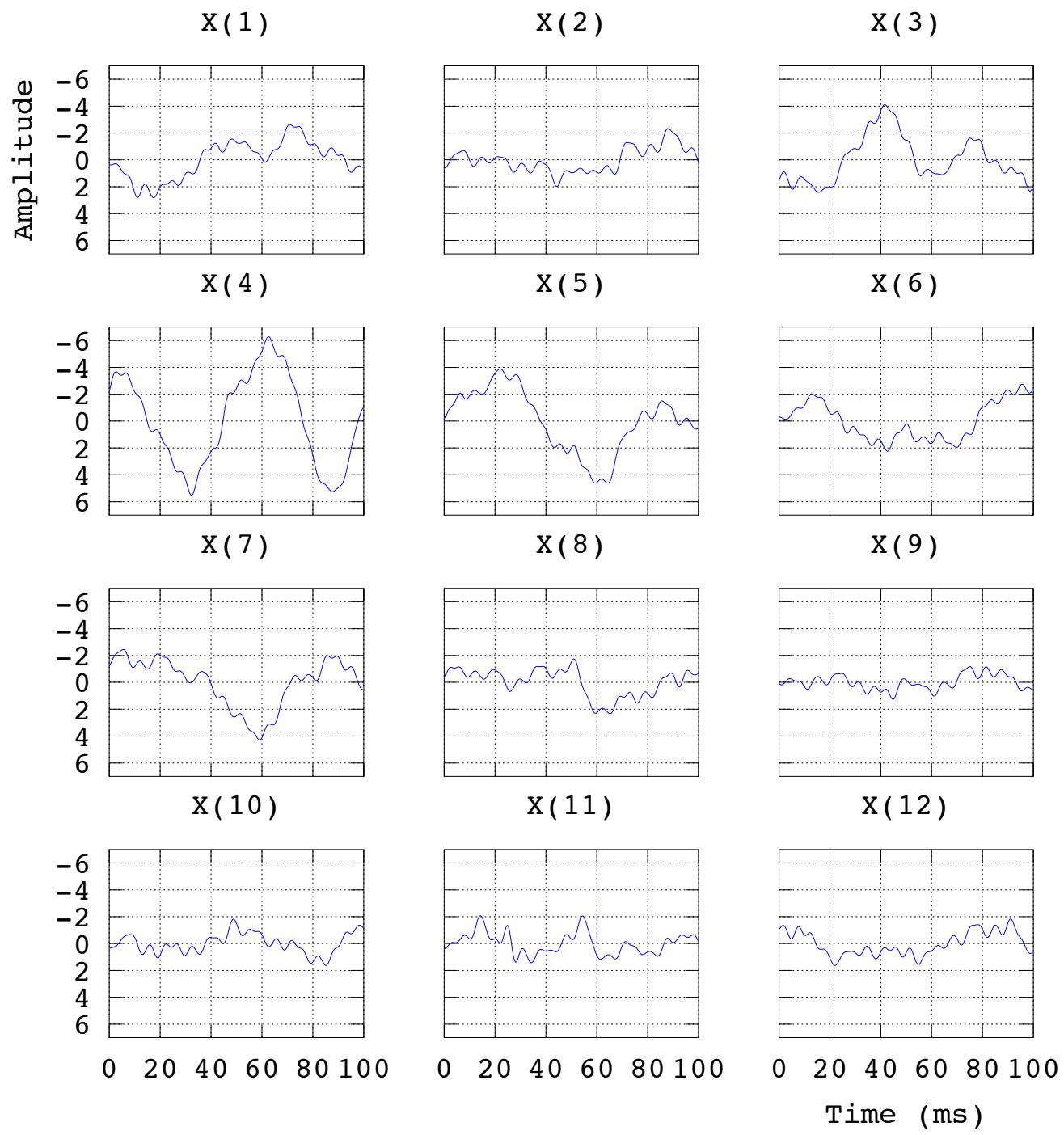

Figure 4.2: A set of twelve successive unrejected recordings for the patient 7 in table 4.1 . 
criterion was used to follow the requirements in the clinical settings i.e., if the amplitude is $0 \mu \mathrm{V}$ or greater than $25 \mu \mathrm{V}$, reject the signal from further analysis fig. 4.2.

\subsubsection{Pre-filtering or Pre-conditioning}

Each of the 12 signals was then preconditioned to remove the $60 \mathrm{~Hz}$ noise and band limit the signal from $50 \mathrm{~Hz}$ to $120 \mathrm{~Hz}$. To achieve this with minimum spectral leakage, the signal was first zero-padded in time domain and a Chebyshev window was applied. The signal was then converted to frequency domain using fast Fourier

transform (FFT) (Madhok et al., 2010; Hu et al., 2011b), where the rectangular window was applied. The inverse FFT will result in a signal devoid of unwanted frequency components and the zero padding can now be removed to get back a signal of $100 \mathrm{~ms}$.

\subsubsection{Eigen Space}

A form of principal component analysis used for blind source separation, called AMUSE was used to analyze the eigen-components of the data set. Experimental evaluations showed that it was always the first three components that contribute to the noise and background EEG in the recordings. Eliminating these components was expected to result in the SSEP from that recording. Figure 4.3 shows the signals before pre-filtering and fig. 4.4 shows the signals after performing the pre-filtering operation. 


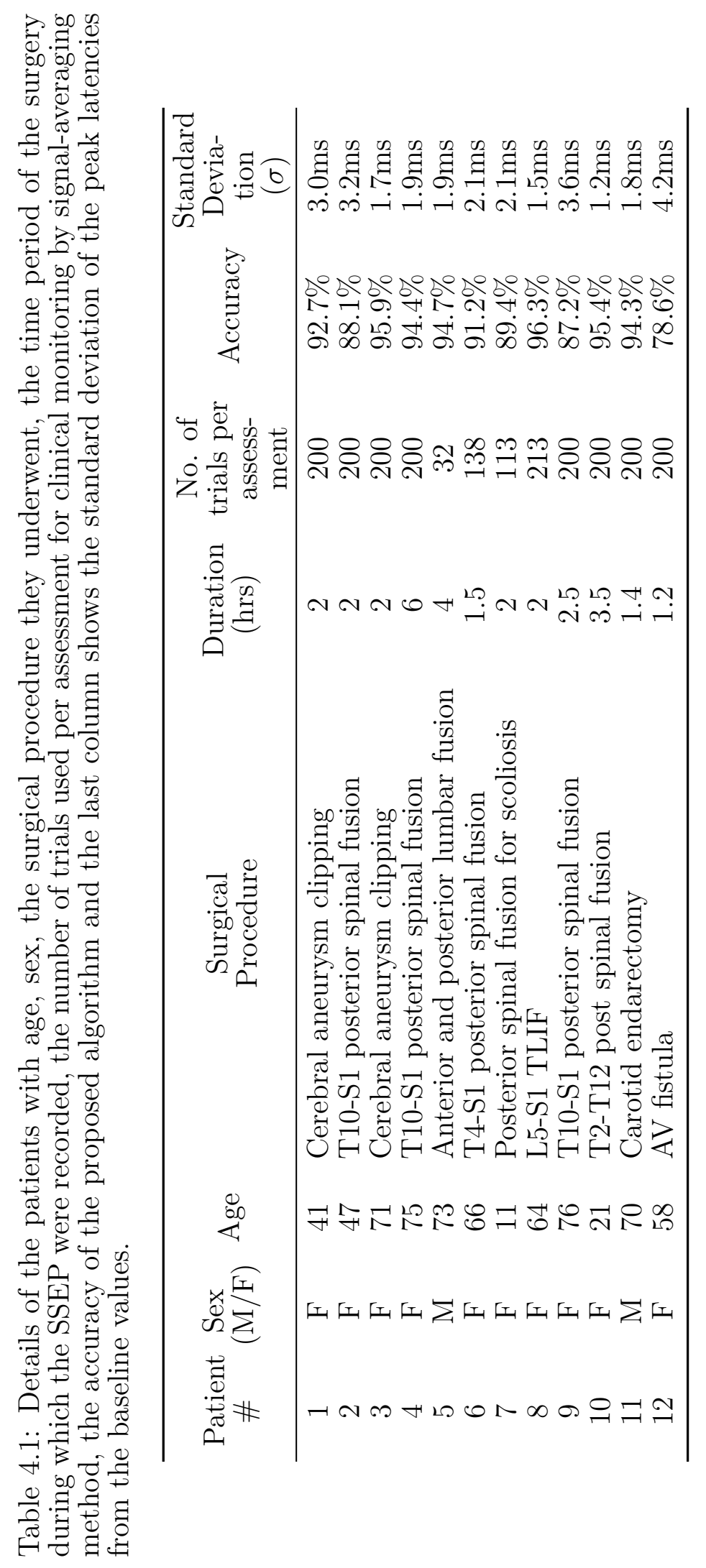



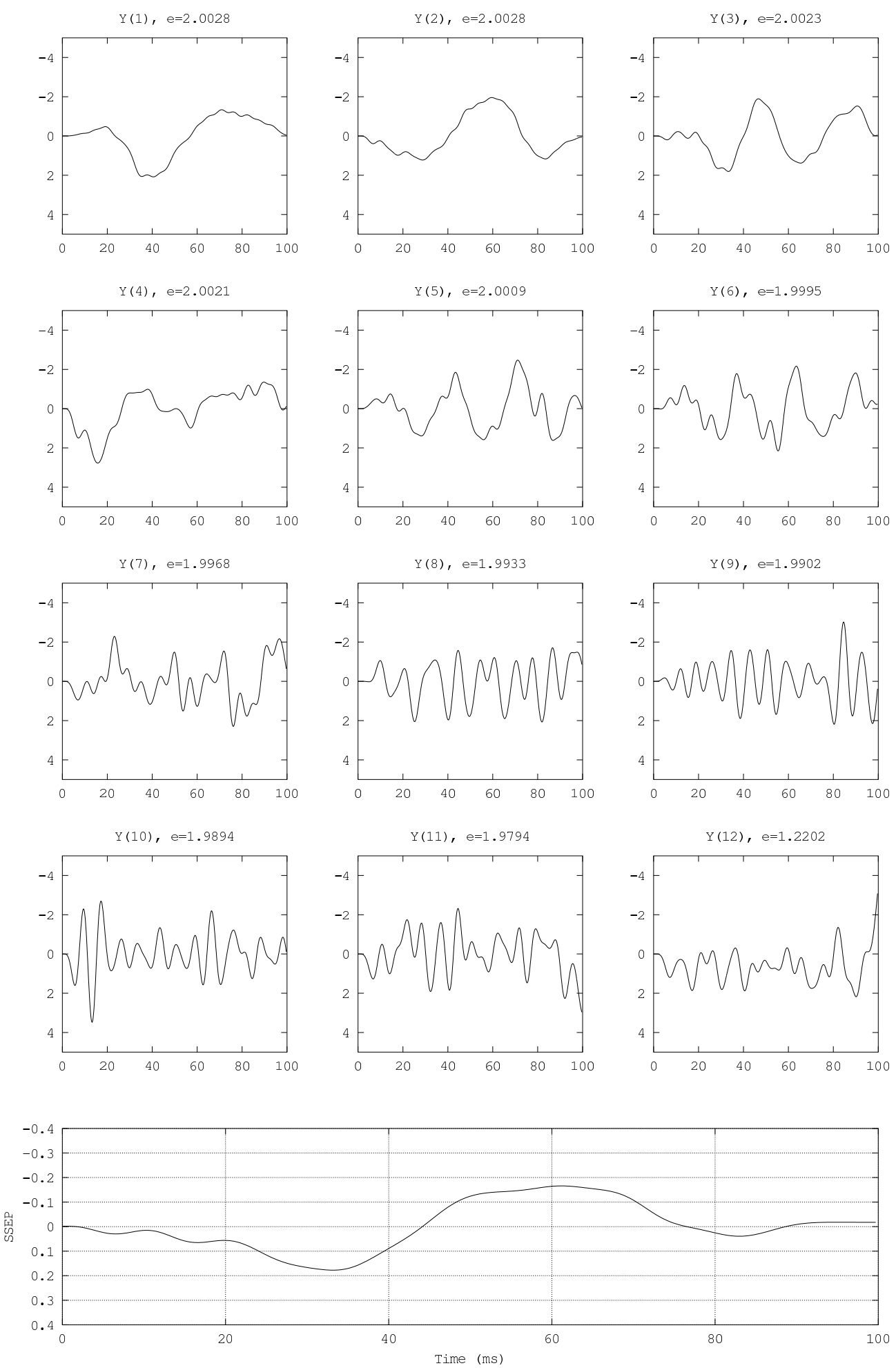

Figure 4.3: The components of a set of 12 sweeps obtained using the AMUSE algorithm when the signals were not pre-conditioned using the Chebyshev window based filtering. The values of ' $\mathrm{e}$ ' are the corresponding eigenvalues for the signal component and have a standard deviation of 0.224 . 

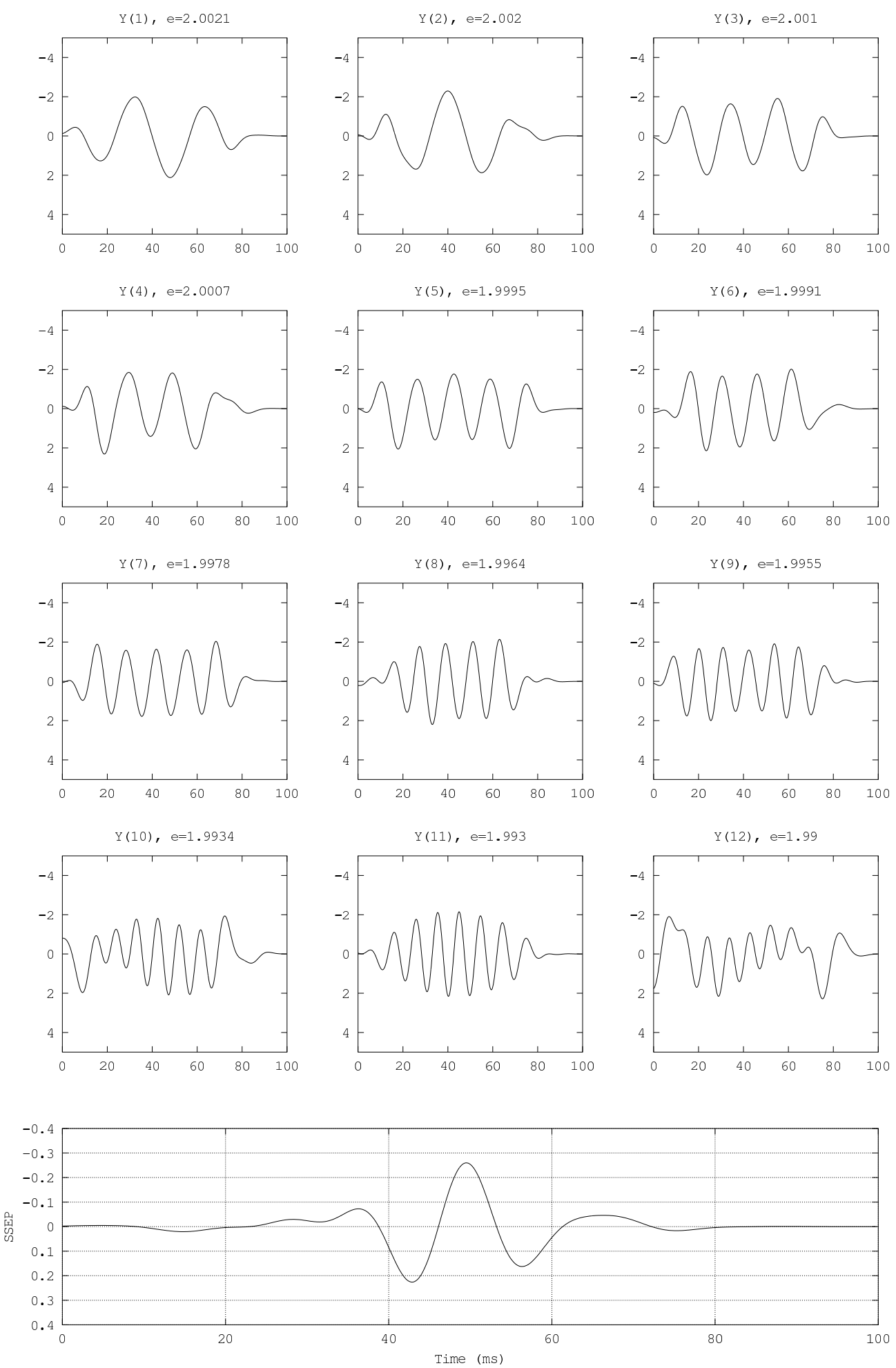

Figure 4.4: The components of a set of 12 sweeps obtained using the AMUSE algorithm when the signals were pre-conditioned using the Chebyshev window based filtering. The values of 'e' for each component are the corresponding eigenvalues and have a standard deviation of 0.0034 . 


\subsubsection{Reconstruction}

These filtered components are used to reconstruct the signals $\mathbf{X}_{r}$ fig. 4.5 that was the SSEP signal from each of the recordings. These 12 reconstructed signals are then averaged in time and the time-locked SSEP signal was obtained.

\subsubsection{Information Extraction}

A Walsh kernel representing the first derivative can then be used to automate the peak detection (Adjouadi et al., 2004) and was used on the extracted SSEP signal to obtain the P37 and N45 peak latencies and the peak-to-peak amplitude fig. 4.6.

\subsection{Results}

The algorithm in this version was robust enough that it would run continuously receiving the EEG recordings, checking for corrupt signals and processing a set of 12 successive good trials to provide with a morphologically and mathematically accurate SSEP waveform. The timeline for the algorithm is represented in fig. 4.7.

\subsubsection{Implementation}

This new algorithm provides an improvement in preserving the morphological characteristics of the SSEP waveforms from earlier versions 1 and 2. The previous algorithm addressed the issue of automated peak detection and presented an algorithm that was designed for the specific intension of being able to verify the waveform by manual inspection. In this new algorithm the objective was to extract automatically the SSEP with a minimum number of sweeps or trials and yet obtain the same 

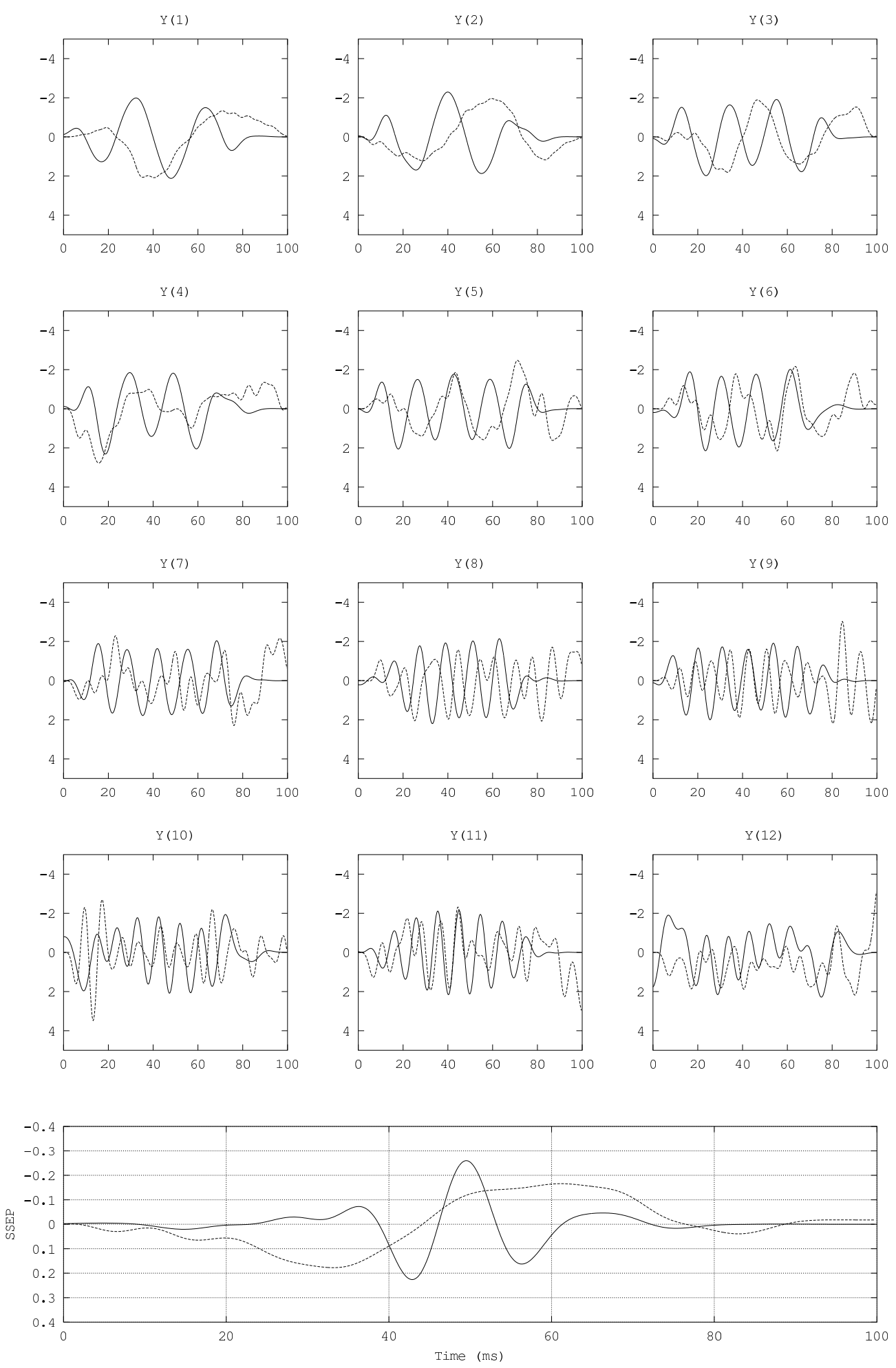

Figure 4.5: A comparison of the components and the extracted SSEP from fig. 4.3 (dotted lines) and fig. 4.4 (solid lines). 


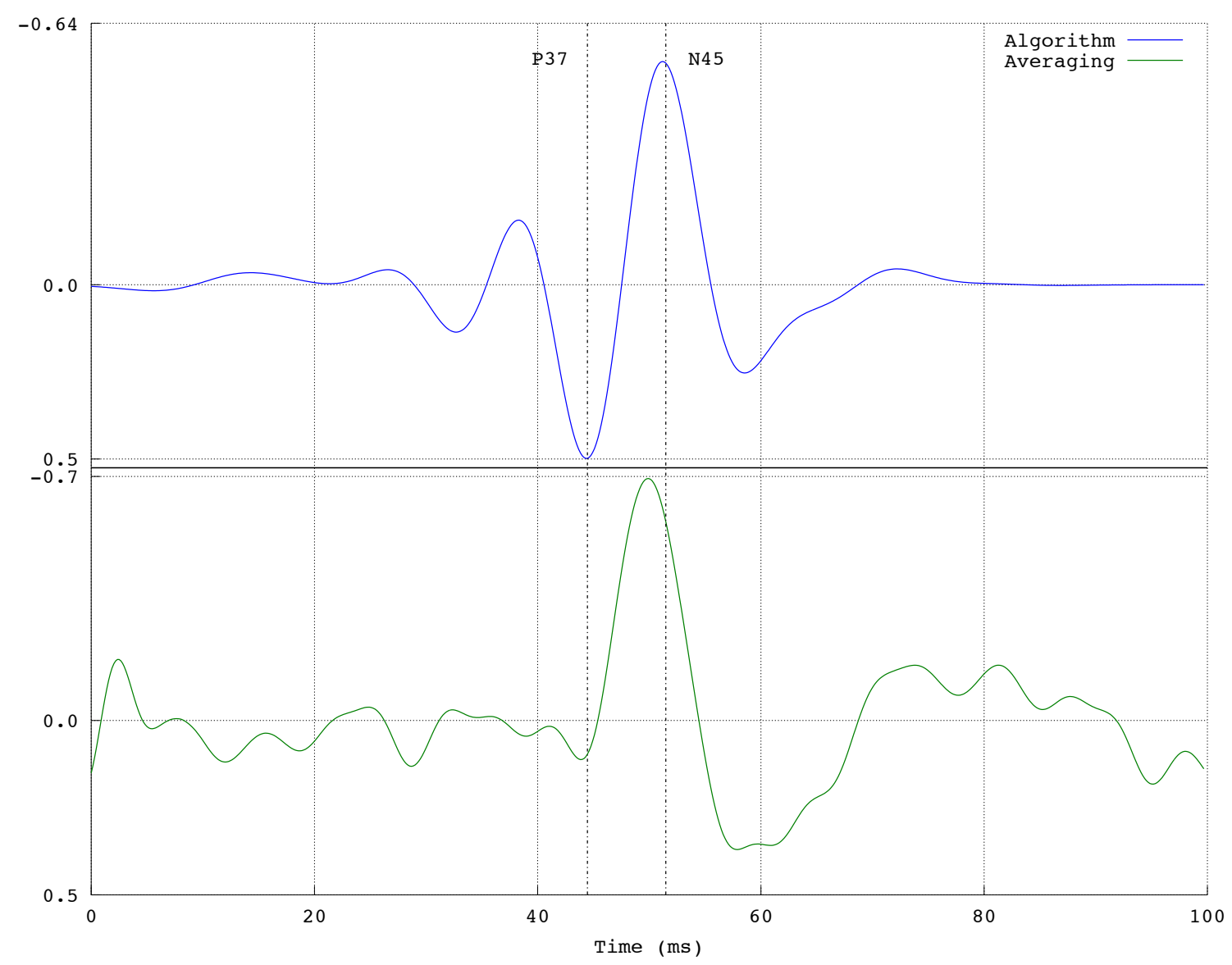

Figure 4.6: Comparison of SSEP extracted by the algorithm using only 12 trials with that obtained using the traditional method of averaging of 113 trials.

morphology as that obtained with the maximum number of trials traditionally used. Significant comfort is reached when time latencies and morphology conform to the true signal.

To weigh the merits of the proposed algorithm, and for illustrative purposes, implementation steps performed on the data for patient number 8 in table 4.1 throughout the 2-hour surgical procedure with all the 479 extracted SSEP waveforms is presented in fig. 4.8. Figure 4.8 displays the extracted waveforms through another similar surgery of patient 7 . The baseline signal is the very first extracted SSEP using the same procedure on the first 12 sweeps and is at the very top of the fig- 
ure. The detected P37 and N45 latencies have been marked with vertical lines for reference. All the 12 tested surgical outcomes were clinically successful without any alarms raised during the surgeries and no neurological damage to the patients were found. To analyze the effectiveness of the algorithm, we check the SNR for the algorithm with that of the clinical approach. The formula used for calculating the SNR was presented warlier in eq. (3.2). The signal is obtained by taking the ensemble average of all the available trials and the noise signal is obtained by alternate addition and subtraction of all the available trials. This method allows us to obtain the SSEP signal and the noise without the SSEP signal at both the input and output of the algorithm (MacDonald et al., 2005). Figure 4.9 shows the SNR improvement achieved by application of the Chebyshev time-frequency windowing method. Table 4.2 summarizes the results from the implementation of the algorithm on all 12 patients.

\subsubsection{False Alarms}

The sensitivity and accuracy of the algorithm can be analyzed based on the number of detections by the algorithm and the actual alarms raised. In the surgeries performed, however, although there were no alarms raised but the proposed algorithm

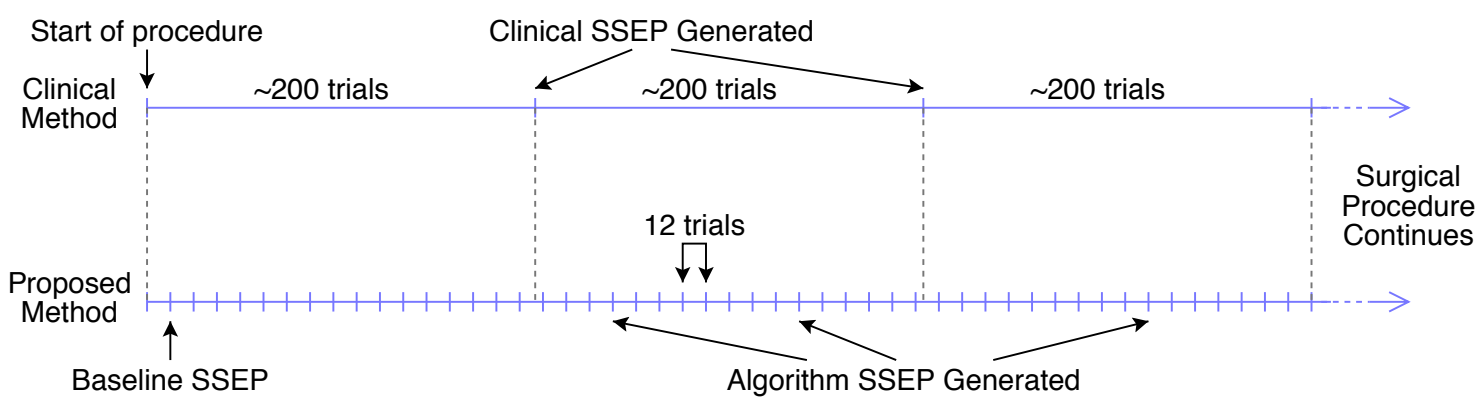

Figure 4.7: Outline of the SSEP recording timeline as compared with the clinical monitoring. By the time one clinical SSEP is obtained, the algorithm version 3 obtaining 12 SSEP signals. 


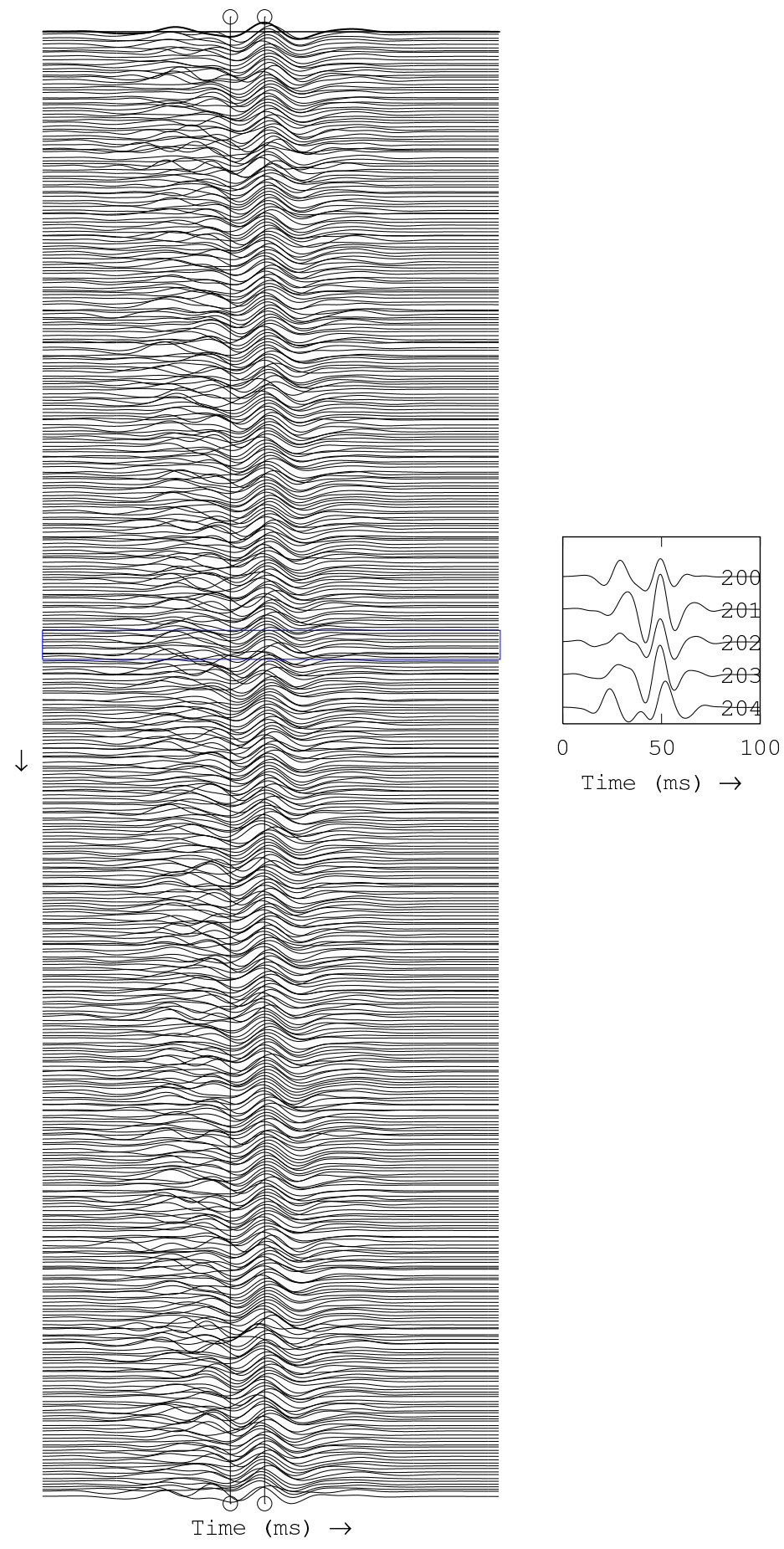

(a) Sequence of $479 \mathrm{SSEPs}$ of $100 \mathrm{~ms}$ extracted in sequence during the two hour-long surgery of patient\# 7 in table 4.1 from the $\mathrm{C}_{3}-\mathrm{C}_{4}$ channel. The baseline signal (earliest SSEP) is on the very top and the last SSEP extracted is at the bottom. Two vertical lines mark the P37 (first one on the left at 41ms) and N45 (second vertical line at $48.5 \mathrm{~ms}$ ) baseline values for reference. Five of the signals in the region marked by a box are displayed in the adjacent plot for illustration. The numbers marked on them are the set numbers from which the SSEP were extracted. 


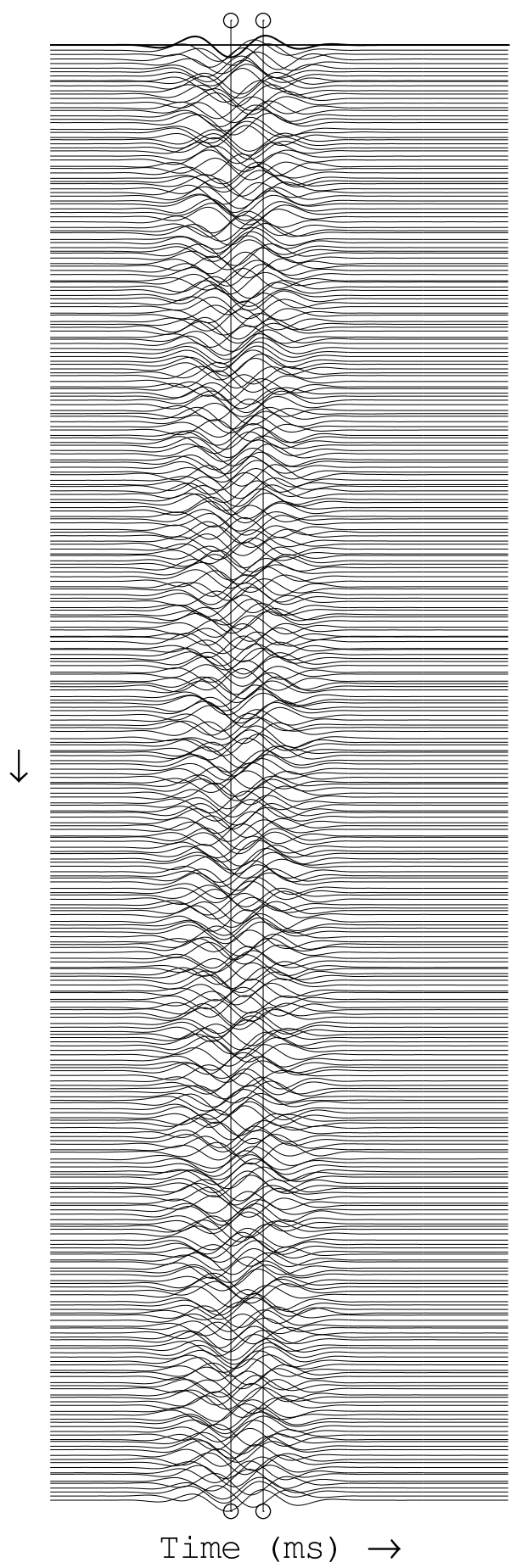

(b) Sequence of 358 SSEPs of $100 \mathrm{~ms}$ extracted in sequence during the two hour-long surgery of patient\# 8 in table 4.1 from the $\mathrm{C}_{3}-\mathrm{C}_{4}$ channel. The baseline signal (earliest SSEP) is on the very top and the last SSEP extracted is at the bottom. Two vertical lines mark the P37 (first one on the left at $39.3 \mathrm{~ms}$ ) and N45 (second vertical line at $46.3 \mathrm{~ms}$ ) baseline values for reference.

Figure 4.8: Sequence of extracted SSEPs of 100ms throughout the surgery. 
Table 4.2: Summary of the results from the implementation of the proposed algorithm showing the average variability in the time latency and amplitude and the total number of false alarms raised for each patient.

\begin{tabular}{cccc}
\hline \multirow{2}{*}{ Patient\# } & \multicolumn{2}{c}{ Average variability $(\%)$} & \multirow{2}{*}{$\begin{array}{c}\text { Number of } \\
\text { false alarms }\end{array}$} \\
\cline { 2 - 3 } & Time latency & Amplitude & 4 \\
1 & 5.1 & 8 & 3 \\
2 & 3.2 & 29 & 4 \\
3 & 1.3 & 22 & 5 \\
4 & 5.8 & 18 & 4 \\
5 & 2.6 & 13 & 2 \\
6 & 1.9 & 12 & 2 \\
7 & 2.3 & 20 & 5 \\
8 & 7.1 & 26 & 4 \\
9 & 3.6 & 19 & 3 \\
10 & 4.1 & 25 & 3 \\
11 & 2.9 & 18 & 2 \\
12 & 2.4 & 15 & $1.6 / \mathrm{hr}$ \\
\hline Average: & 3.3 & 19 & \\
\hline
\end{tabular}

did raise alarms that can be considered as false alarms. The false alarms detected were due to peak-to-peak amplitude variation of more than $50 \%$, fig. 4.10. It was shown in table 4.2 that there were, on an average, 1.6 false alarms per hour. If the false alarms are quantized as the percentage of false alarms occurring per subject for every set of 12 trials used to extract the SSEP, we obtain an average of $0.09 \%$ of false alarms for this specific presented study. It must be noted that the extracted SSEP signals are obtained every twelve trials and hence the short-term SSEP changes that go unnoticed by the conventional averaging method are easily acquired using the algorithm. Hence, for a true positive, the changes must persist for 12 successive SSEP extracted using the algorithm. No such case was observed in the study confirming that no alarms were raised during the procedures.

As an example, the implementation results for patient 10 from table 4.1 are presented in table 4.3. Another example of consistency through a surgical procedure is 
displayed in fig. 4.11. 
Table 4.3: Amplitude and time latency consistency for patient 10 from table 4.1 obtained using the final version of algorithm. The first recording is considered the baseline value and hence no variation value. The values in red indicate the occurrence of false alarms.

\begin{tabular}{|c|c|c|c|c|}
\hline $\begin{array}{c}\text { Patient } \\
\#\end{array}$ & Time & $\begin{array}{l}\text { Electrode } \\
\text { channel }\end{array}$ & $\begin{array}{c}\text { Latency } \\
\text { variation }\end{array}$ & $\begin{array}{c}\text { Amplitude } \\
\text { variation }\end{array}$ \\
\hline \multirow{44}{*}{10} & 11:13 AM & \multirow{44}{*}{$\mathrm{C}_{\mathrm{Z}}-\mathrm{F}_{\mathrm{Z}}$} & - & - \\
\hline & 11:20 AM & & $4.09 \%$ & $6 \%$ \\
\hline & 11:21 AM & & $4.91 \%$ & $36 \%$ \\
\hline & $11: 22 \mathrm{AM}$ & & $4.36 \%$ & $50 \%$ \\
\hline & $11: 23 \mathrm{AM}$ & & $4.09 \%$ & $0 \%$ \\
\hline & $12: 21 \mathrm{PM}$ & & $4.91 \%$ & $30 \%$ \\
\hline & $12: 33$ PM & & $2.18 \%$ & $31 \%$ \\
\hline & $12: 35$ PM & & $4.09 \%$ & $46 \%$ \\
\hline & $12: 49$ PM & & $3.54 \%$ & $29 \%$ \\
\hline & $12: 52 \mathrm{PM}$ & & $4.63 \%$ & $6 \%$ \\
\hline & $12: 55 \mathrm{PM}$ & & $11.4 \%$ & $5 \%$ \\
\hline & 12:56 PM & & $4.91 \%$ & $1 \%$ \\
\hline & 12:57 PM & & $1.09 \%$ & $12 \%$ \\
\hline & 12:59:15 PM & & $3.82 \%$ & $21 \%$ \\
\hline & 12:59:39 PM & & $4.36 \%$ & $46 \%$ \\
\hline & 1:00 PM & & $7.90 \%$ & $37 \%$ \\
\hline & 1:01 PM & & $1.91 \%$ & $40 \%$ \\
\hline & 1:11 PM & & $7.36 \%$ & $50 \%$ \\
\hline & $1: 13 \mathrm{PM}$ & & $2.45 \%$ & $11 \%$ \\
\hline & $1: 14 \mathrm{PM}$ & & $1.36 \%$ & $32 \%$ \\
\hline & $1: 16 \mathrm{PM}$ & & $3.00 \%$ & $23 \%$ \\
\hline & $1: 18$ PM & & $1.91 \%$ & $22 \%$ \\
\hline & 1:19 PM & & $4.91 \%$ & $25 \%$ \\
\hline & $1: 20 \mathrm{PM}$ & & $3.27 \%$ & $29 \%$ \\
\hline & 1:21 PM & & $6.54 \%$ & $11 \%$ \\
\hline & 1:22:09 PM & & $4.09 \%$ & $23 \%$ \\
\hline & $1: 22: 58 \mathrm{PM}$ & & $3.00 \%$ & $2 \%$ \\
\hline & 1:24 PM & & $8.17 \%$ & $26 \%$ \\
\hline & $1: 26 \mathrm{PM}$ & & $2.18 \%$ & $41 \%$ \\
\hline & 1:31 PM & & $6.54 \%$ & $26 \%$ \\
\hline & 1:56:15 PM & & $5.45 \%$ & $21 \%$ \\
\hline & $1: 56: 40 \mathrm{PM}$ & & $0.82 \%$ & $35 \%$ \\
\hline & 1:58 PM & & $3.00 \%$ & $38 \%$ \\
\hline & 1:59 PM & & $0.82 \%$ & $2 \%$ \\
\hline & 2:00 PM & & $7.08 \%$ & $51 \%$ \\
\hline & 2:02 PM & & $1.91 \%$ & $5 \%$ \\
\hline & 2:03 PM & & $3.54 \%$ & $47 \%$ \\
\hline & 2:05 PM & & $1.91 \%$ & $23 \%$ \\
\hline & 2:06 PM & & $6.00 \%$ & $32 \%$ \\
\hline & 2:10 PM & & $3.54 \%$ & $37 \%$ \\
\hline & 2:11 PM & & $2.18 \%$ & $22 \%$ \\
\hline & $2: 20 \mathrm{PM}$ & & $6.54 \%$ & $3 \%$ \\
\hline & $2: 21 \mathrm{PM}$ & & $2.45 \%$ & $44 \%$ \\
\hline & $2: 22 \mathrm{PM}$ & & $1.91 \%$ & $23 \%$ \\
\hline
\end{tabular}




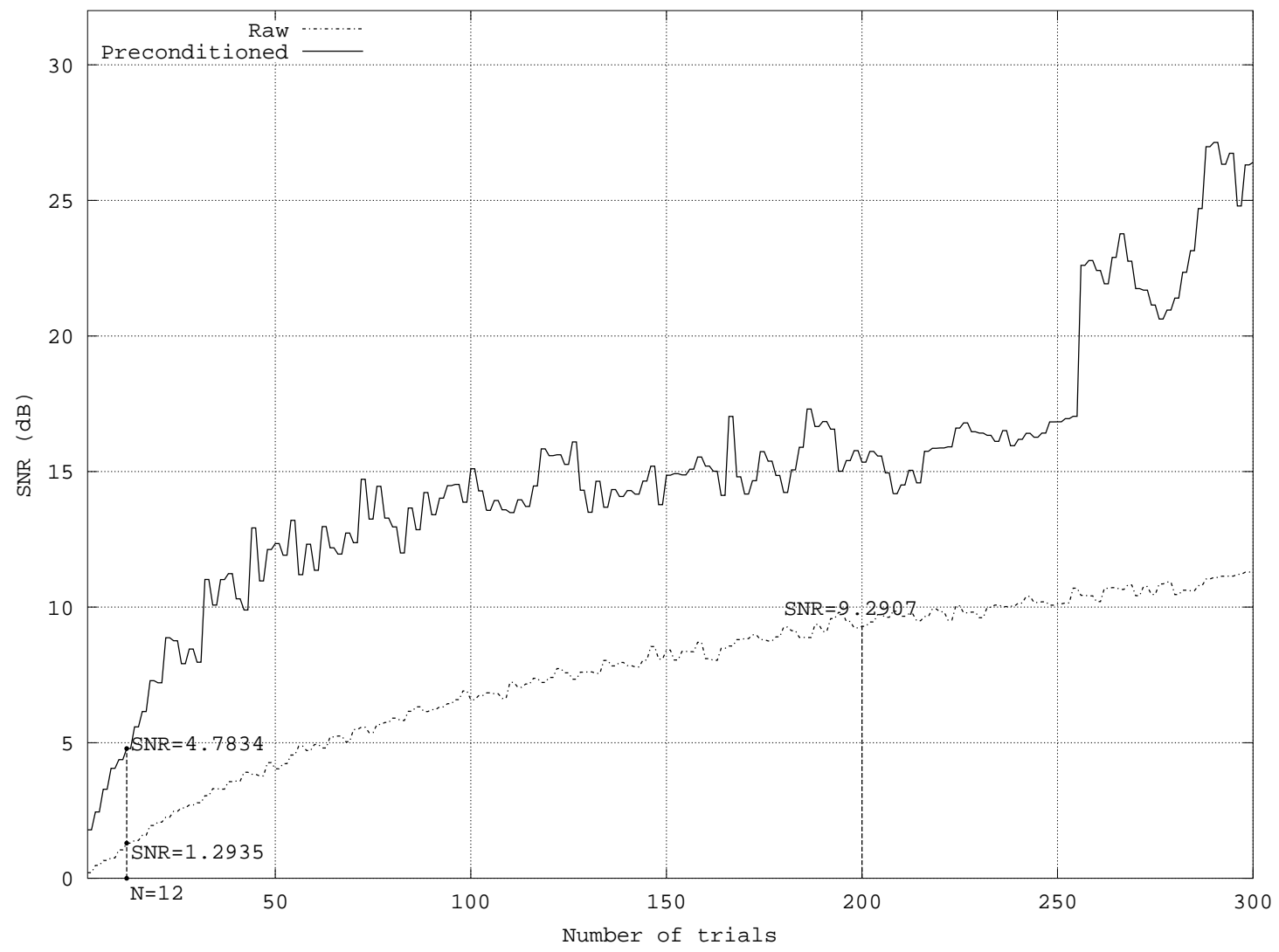

Figure 4.9: The signal to noise ratio (SNR) comparison for clinical SSEP signals and post-processed signals. SNR values are provided for the two cases when 12 trials are used and for 200 raw trials. 


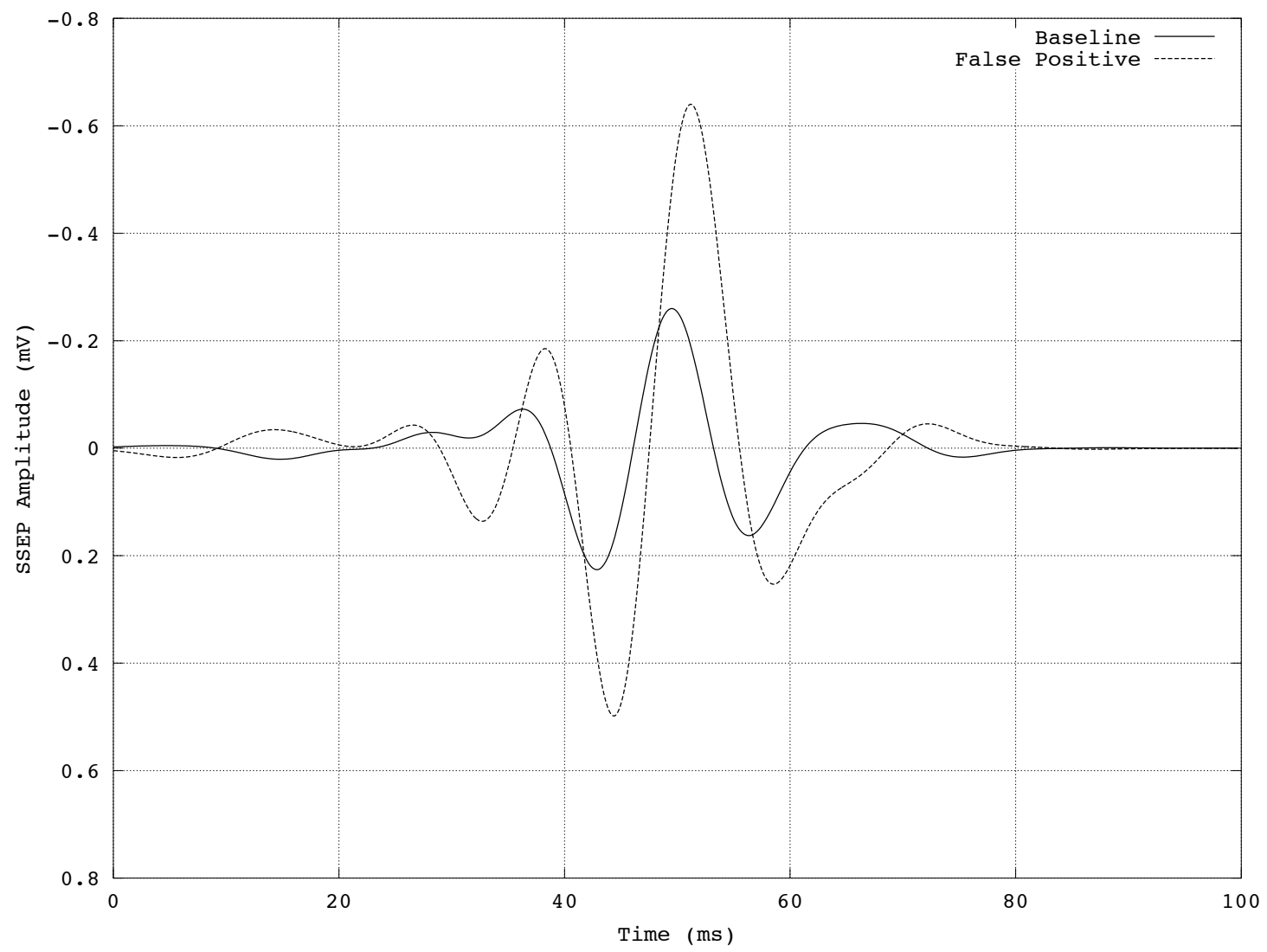

Figure 4.10: Comparison of a false positive SSEP signals as detected due to high amplitude variation for patient 8 in table $4.1 \mathrm{C}_{3}-\mathrm{C}_{4}$ montage at $23 \mathrm{~min}$ through the procedure with the baseline SSEP. 


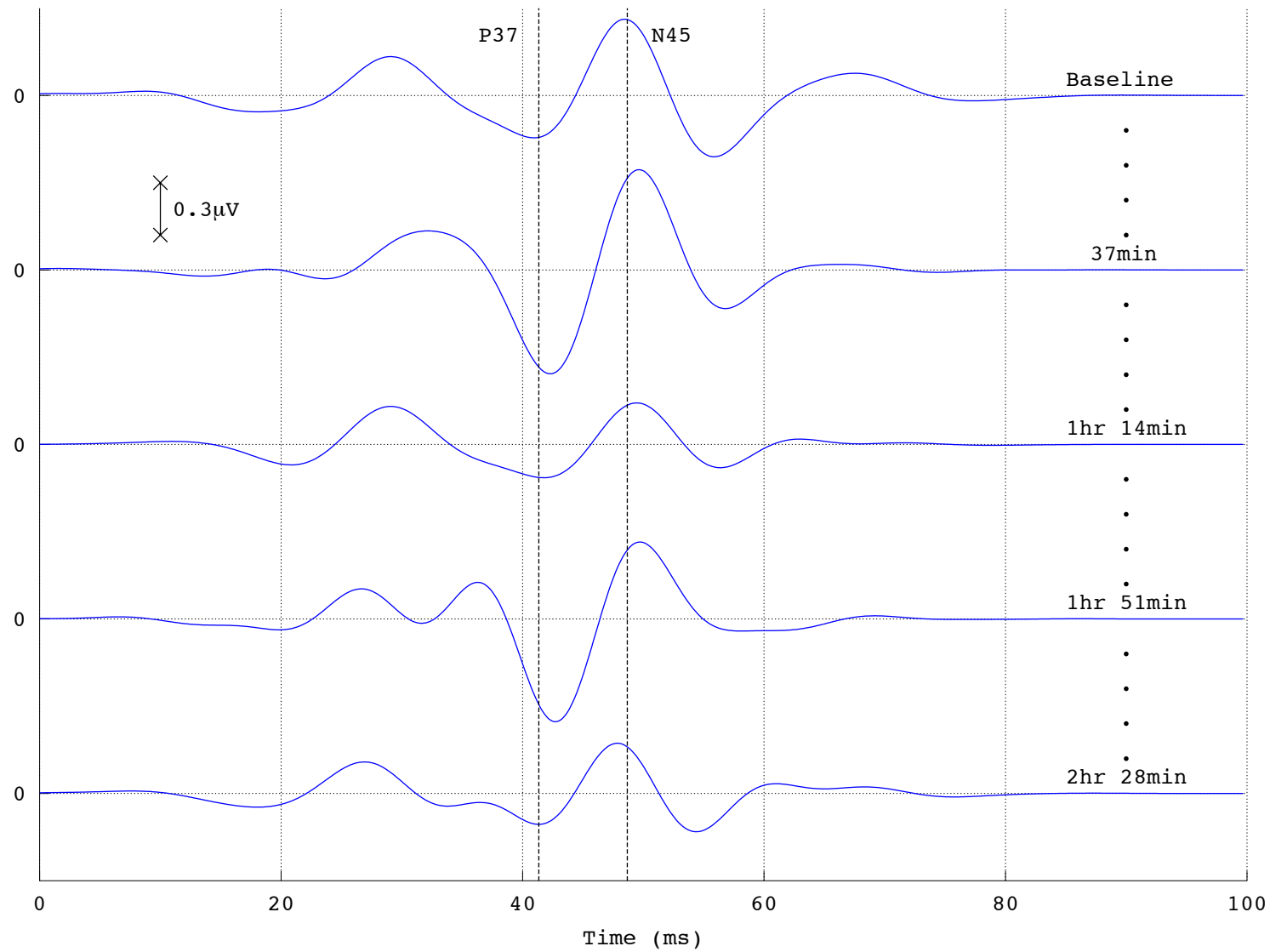

Figure 4.11: The baseline signal and tSSEP signals at different times shown for comparison. The vertical markers are for average P37 and N45 peak latencies for Patient\# 9 from table 4.1. Note that the vertical axis is inverted, with positive direction going downward following the convention of displaying SSEP. 


\section{CHAPTER 5 \\ CONCLUSIONS AND FUTURE WORK}

The new algorithms developed and presented in the dissertation attempted to address the hypotheses proposed and questions raised in this study. The primary objective was to understand the characteristics of an SSEP and classify it from background brain activity, a task that is critical in monitoring spinal surgeries.

\subsection{Conclusions from algorithm versions}

\subsubsection{Version 1}

The eigen-decomposition process helped reduce significantly the number of trials, a clinical outcome that is highly desirable, and allowed for a thorough assessment that delineated signal trend from noise. The use of the Walsh operator proved highly effective in detecting the evoked potential peak latencies using an optimal number of trials (in this study, limited to 10). The algorithm no longer depends on the signal morphology and automates the process from selection of a minimum number of trials based on their frequency response, applying the algorithm on them and using the unique Walsh transformation method to automatically indicate the SSEP response and the peak latencies. The results from the automated detection scheme and interpretation of the characteristic peak using the Walsh operation coincide with expertsâẮ́ opinions.

For all of the cases the noted average misalignment between the clinical peak latencies and those obtained using the proposed algorithm, including both bipolar channels, was $3.38 \mathrm{~ms}$ and $4.3 \mathrm{~ms}$ for P37 and N45, respectively. It should be stated that when such misalignments happen during a surgical procedure, even when a 
maximum number of trials is used, clinicians select one of the two channels that is viewed as more representative of the SSEP morphology.

The Walsh operator proved highly effective in identifying the SSEP occurrence even when the morphology of the signal is quite different from that obtained at a much higher number of trials. In the automated process, a thorough analysis yielded a better mathematical assessment of the noise signal involved in evoked potentials. Since there are no intensive mathematical operations involved in the algorithm, it can be feasibly realized in hardware form and/or integrated in the present systems to establish a very valuable tool in IONM.

\subsubsection{Version 2}

Consistency in the results is also satisfied by the current algorithm for SSEP detection, which demonstrated an average percentage deviation of $4.47 \pm 3.33 \%$ and $3.73 \pm 3.92 \%$ in the detection of the time latencies of the P37 and N45 peaks, respectively, using the $\mathrm{C}_{3}-\mathrm{C}_{4}$ channel; and $4.60 \pm 4.18 \%$ and $3.30 \pm 2.46 \%$ when the $\mathrm{C}_{\mathrm{Z}}-\mathrm{F}_{\mathrm{Z}}$ channel is used. Also, the algorithm was able to get consistent results in the detection of the peak-to-peak amplitude of the SSEP signals. The algorithm demonstrated an average percentage deviation of $19.81 \pm 13.84 \%$ and $23.97 \pm 24.23 \%$ in the calculation of the SSEP peak-to-peak amplitude for the $\mathrm{C}_{3}-\mathrm{C}_{4}$ and $\mathrm{C}_{\mathrm{Z}}-\mathrm{F}_{\mathrm{Z}}$ channels,

respectively. These values are below the clinically approved levels for deviation of $10 \%$ for the time latencies and $50 \%$ for the peak-to-peak amplitude. What is even more important is the fact that the results were found to be consistent throughout the surgical procedure for all the patients considered.

During surgery, the decisions are based on either one of the channels: $\mathrm{C}_{3}-\mathrm{C}_{4}$ and 
Table 5.1: Summary of results showing the average percentage error and percentage deviation in SSEP detection using either $\mathrm{C}_{3}-\mathrm{C}_{4}$ channel or $\mathrm{C}_{\mathrm{Z}}-\mathrm{F}_{\mathrm{Z}}$ channels. The logical OR function of the two channels is denoted by symbol (V).

\begin{tabular}{|c|c|c|c|c|c|}
\hline \multirow{2}{*}{ Electrode } & \multicolumn{2}{|c|}{ \% Error } & \multicolumn{3}{c|}{ \% Deviation from baseline } \\
\cline { 2 - 6 } & P37 & N45 & P37 & N45 & p-p Amp \\
\hline \hline $\mathrm{C}_{3}-\mathrm{C}_{4}$ & $3.70 \pm 3.04$ & $4.03 \pm 3.28$ & $4.47 \pm 3.33$ & $3.73 \pm 3.92$ & $19.81 \pm 13.84$ \\
$\mathrm{C}_{\mathrm{Z}}-\mathrm{F}_{\mathrm{Z}}$ & $3.51 \pm 3.68$ & $4.07 \pm 3.35$ & $4.60 \pm 4.18$ & $3.30 \pm 2.46$ & $23.97 \pm 24.23$ \\
$\left(\mathrm{C}_{3}-\mathrm{C}_{4}\right) \mathrm{V}$ & $1.91 \pm 1.38$ & $2.11 \pm 1.82$ & $2.46 \pm 1.84$ & $1.88 \pm 1.26$ & $11.14 \pm 10.44$ \\
$\left(\mathrm{C}_{\mathrm{Z}}-\mathrm{F}_{\mathrm{Z}}\right)$ & & \multicolumn{3}{|c}{} \\
\hline
\end{tabular}

$\mathrm{C}_{\mathrm{Z}}-\mathrm{F}_{\mathrm{Z}}$. The clinicians select one of the two channels that is viewed as more representative of the SSEP morphology. Taking this into consideration, table 5.1 presents the results of a logical 'OR' function that represents the results obtained by using the results from the electrode that provided the better results. Table 5.1 thus provides an estimate of the best-case scenario where the electrode that provided a better detection of the SSEP is picked for the analysis. Please note that patient 4 was not included in the analysis for this $\mathrm{OR}$ function analysis since the $\mathrm{C}_{3}-\mathrm{C}_{4}$ data were not available. The results in table 5.1 demonstrate that the algorithm is capable of detecting the P37 and N45 peak latencies with a very high accuracy of about $98 \%$ in situations when the best electrode channel is chosen. As such, the average percentage deviations from the baseline are also reduced by approximately a factor of 2 .

Various other techniques have addressed the problem of SSEP detection with the aim of reducing the number of trails. It was shown that 50 input trials can provide similar performance as compared to the ones obtained using 1000 trials (Lam et al., 2005). Single trial determination of SSEP using a combination of EEG-based probabilistic independent component analysis and wavelet filtering had been presented (Hu et al., 2011b). Another study had also shown the determination of SSEPs using 80 trials which provided an average accuracy of $0.75 \% \pm 1.12$ and $0.86 \% \pm 0.37$ of the P37 
and N45 peaks (Hu et al., 2009). Liu et al. presented an independent component analysis (ICA) method for the detection of SSEP monitoring using 300 simulated trials (Liu et al., 2007).

The developed algorithm provides a simple yet precise solution toward the detection of the SSEP waveform from a significantly reduced number of trials. Instructive steps were consolidated from empirical evaluations and observations to ensure that noisy signals were removed using key pre-processing steps. These included (1) a statistical threshold on the amplitude of the signals which removed a priori any trial whose change is one standard deviation higher than the mean of the standard deviations of all 30 channels; and on the remaining trials, (2) the use of a fouth-order Butterworth notch filter with a stop band from $55 \mathrm{~Hz}$ to $65 \mathrm{~Hz}$ that removed the ubiquitous $60 \mathrm{~Hz}$ noise; (3) the use of a fifth-order Butterworth low-pass filter with a cut-off frequency of $300 \mathrm{~Hz}$ that served as a notch filter to remove any unwarranted high-frequency components, which in the context of the desired SSEP signal are considered as noise; and (4) the use of a moving average filter with a 25-point window that served as a smoothing filter.

With all these initial preprocessing steps properly executed, a significant contribution of this algorithm is in introducing patient-specific Gaussian templates that envelop the SSEPs, delineating them clearly from other portions of the signal and any remnant artifacts embedded within a given trial.

\subsubsection{Version 3}

The algorithm is able to exploit multiple characteristics of the SSEP simultaneously viz., the morphology of the signal in time domain, the frequency characteristics and 
the eigen space components that contribute to the signal. The AMUSE algorithm is able to separate the SSEP with the background brain activity but is not able to preserve the signal morphology in the time domain.

The novelty of the approach was the use of Chebyshev windowing for preconditioning the signal before linear filtering for $60 \mathrm{~Hz}$ notch and low pass filter. The windowing method considerably improved the SNR of the raw signals (fig. 4.9) but still required 50 or more number of trials to obtain the SNR close to practical values (Goryawala et al., 2012; Hu et al., 2003). Thus enhancing the prospects for the AMUSE algorithm to better classify the signal components. The preconditioning improved the SNR by about $4 \mathrm{~dB}$ in the first twelve trials. The important contribution was the improvement in SNR by preconditioning in a much reduced number of trials and coupled with the AMUSE algorithm to better extract the SSEP preserving the morphology of the signal.

When linear filtering was performed without this pre-conditioning, the signal components obtained in the AMUSE algorithm did not show a trend in the signal-noise power characteristics and the standard deviation of the eigenvalues was high, typically of the order of 0.12 . Though the extracted SSEP was clearly identifiable (fig. 4.3), the signal showed spreading in time and was reconstructed from the components with the higher eigenvalues. With the windowing, however, as was illustrated in fig. 4.4, the eigenvalues were more organized with noise power concentrated in the components with the higher eigenvalues. This is because the Chebyshev window limits the spectral power leakage and concentrates them in the frequency band of interest thus improving the contrast of the signal. Figures 4.3 and 4.4 depicted the same set of 12 sweeps that form the $5^{\text {th }}$ SSEP of the fig. 4.8 , the former without the Chebyshev windowing and the latter with Chebyshev windowing. These two 
results were superimposed and displayed in fig. 4.6 for comparative purposes.

The AMUSE algorithm performed in the same manner in both cases and was able to separate the signal components attributing to the background EEG. However, with no signal preconditioning, the signal components showed (1) wide variation with respect to their corresponding eigenvalues and (2) wide amplitude variations; whereas with conditioning, the components showed (1) close and evenly distributed eigenvalues and (2) the signal power of the components was relatively uniform.

It was also interesting to observe that the signal components with and without the preconditioning showed regions of very high correlation. The signal preconditioning thus helped to improve the contrast between the signal components that the AMUSE algorithm extracts. These results provide the impetus to proceed with an exhaustive number of implementations for further confirmation.

The algorithm was tested on 12 patients and the results were presented in table 4.2 . The algorithm was successful in extracting the SSEP signals throughout all surgeries. These results were obtained using only 12 trials that passed the exclusion criterion and checks for the consistencies and any causes for false alarms. The 12 surgical cases did not have any alarms raised during the clinical procedures. The algorithm, however, did raise alarms and these were termed as 'false alarms'. This fact assures us that the algorithm is capable of detecting and raising alarms. As such, the accuracy of the algorithm was defined as

$$
\% \text { Accuracy }=\left(1-\frac{\text { no.of false alarms }}{\text { no.of SSEP signals }}\right) \times 100
$$

The sensitivity and accuracy of the algorithm can be analyzed based on the number 
of detections by the algorithm and the actual alarms raised. In the surgeries that were performed, no alarms were raised throughout; however the algorithm did raise alarms that were considered as false alarms. It was shown in table 4.2 that there are, on an average, 1.6 false alarms per hour. If the false alarms are quantized as the percentage of false alarms occurring per subject for every set of 12 trials used to extract the SSEP, we obtain an average of $0.09 \%$ of false alarms.

It is very important to note that since the extracted SSEP signals are obtained every twelve trials and hence the short-term SSEP changes that would have otherwise gone unnoticed by the conventional averaging method are easily detected when using the proposed algorithm. Hence, for a true positive, the changes must persist for 12 successive SSEP signals extracted using the algorithm. No such case was observed in the study confirming that no alarms were raised during the procedures.

On an average, the algorithm raised 1.6 false alarms per hour and presented an accuracy of $91.5 \%$. An example of a typical SSEP extracted using the proposed algorithm for a given subject at five different instances of time during the surgery was shown in fig. 4.10. Note the merits of using Chebyshev as means to preserve the morphology of the SSEP signal.

\subsection{Discussion}

In version 1 , it was observed that even though the automation scheme was shown to be viable, the IIR filtering applied at the very end might not give an SSEP true to its morphological characteristics to be observed by experienced eye (Vedala et al., 2012b). We observed, in the final version 3, that such filtering is more beneficial when applied prior to eigen-space filtering. Conventional systems also adopt a sim- 
ilar approach of filtering using moving average type filters after signal averaging. These are linear phase filters. Hence, we choose Chebyshev time windowing prior to eigen-space filtering. This has the merit of limiting power leakage of the frequency components of SSEP to adjacent frequencies. A rectangular window in the frequency domain eliminated undesired frequency components and preserved those frequencies that contribute most to the SSEP signal. It is also effective in removing the $60 \mathrm{~Hz}$ noise introduced by the electrical equipment in the vicinity.

It is a long known fact that EEG sources are non-stationary (Delorme et al., 2012), however, during the 100ms time windows, as is common in IONM, the EEG sources are considered quasi-stationary (Kaplan et al., 2005; Zygierewicz et al., 2006), and hence the AMUSE algorithm was able to identify only the known stationary SSEP component. On the other hand, the use of Chebyshev time window a priori made certain that any other stationary and quasi-stationary components are eliminated.

The current algorithms assume that the SSEP does not change during the time when the 200 or more trials are recorded and rely on the frequency characteristics of the signal rather than the SSEP morphology in time domain. Other proposed approaches also tend to focus on only one aspect of the SSEP characteristics, mainly in the frequency domain. The present approach in retrospect focuses on preserving the time domain features of SSEP and eliminates the inter-trial variance and extracts the SSEP while considering the ongoing brain activity.

To ascertain the validity of the algorithm, we present the results of the implementation of the algorithm on 12 surgical procedures that lasted anywhere between 1.5 and 6hrs. These surgeries were successful with no resulting neurophysiological effects. The algorithm also ascertained that the peak latencies and peak-to-peak amplitudes 
are within the required limits to prove consistency. The algorithm, however, raised 1.6 false alarms per hour. This can be considered as a good sign because this proves that the algorithm is capable of detecting a true alarm, although not experienced in these surgeries.

\subsection{Future Study and Recommendations}

\subsubsection{Exhaustive study}

The data available for the study contained no true positive cases and hence, a critical study involving sensitivity of the algorithm could not be presented. Future study should involve the following:

(a) Implementation of algorithm on patients where a true clinical alarm was indeed raised in order to assess the algorithm's accuracy and sensitivity in detecting true positives.

(b) Implementation of algorithm on SSEP waveforms from other extremities (e.g. ulnar nerve).

(c) Implementation of algorithm on expanded subject cases involving appropriate statistics that include gender, height, type of surgical procedure and the type of anaesthesia used.

\subsubsection{Practical implementation}

The simplistic nature of the final algorithm (version 3) makes it possible for its potential implementation in both hardware and software forms. The present IONM software systems can readily incorporate the algorithm and provide the benefits 
readily. The hardware implementation provides many advantages. Primarily, implementation in hardware form would minimize both the setup process and the processing requirements in the operating theater, but at the same time providing with the same benefits of a software based system. 


\section{REFERENCES}

Adjouadi, M., Cabrerizo, M., Ayala, M., Sanchez, D., Yaylali, I., Jayakar, P., and Barreto, A. (2005). Detection of interictal spikes and artifactual data through orthogonal transformations. Journal of clinical neurophysiology : official publication of the American Electroencephalographic Society, 22(1):53-64.

Adjouadi, M. and Candocia, F. (1994). A stereo matching paradigm based on the Walsh transformation. IEEE Transactions on Pattern Analysis and Machine Intelligence, 16(12):1212-1218.

Adjouadi, M., Candocia, F., and Riley, J. (1996). Exploiting Walsh-based attributes to stereo vision. IEEE Transactions on Signal Processing, 44(2):409-420.

Adjouadi, M., Sanchez, D., Cabrerizo, M., Ayala, M., Jayakar, P., Yaylali, I., and Barreto, A. (2004). Interictal spike detection using the Walsh transform. IEEE transactions on bio-medical engineering, 51(5):868-72.

Bai, O., Nakamura, M., Nagamine, T., and Shibasaki, H. (2001). Parametric modeling of somatosensory evoked potentials using discrete cosine transform. IEEE Transactions on Biomedical Engineering, 48(11):1347-51.

Candocia, F. and Adjouadi, M. (1997). A similarity measure for stereo feature matching. IEEE Transactions on Image Processing, 6(10):1460-4.

Celesia, G. G. (1979). Somatosensory evoked potentials recorded directly from human thalamus and Sm I cortical area. Archives of neurology, 36(7):399-405.

Cichocki, A., Shishkin, S. L., Musha, T., Leonowicz, Z., Asada, T., and Kurachi, T. (2005). EEG filtering based on blind source separation (BSS) for early detection of Alzheimer's disease. Clinical neurophysiology : official journal of the International Federation of Clinical Neurophysiology, 116(3):729-37.

Crespo-Garcia, M., Atienza, M., and Cantero, J. L. (2008). Muscle artifact removal from human sleep EEG by using independent component analysis. Annals of Biomedical Engineering, 36(3):467-75.

Crittenden, M. D. (2001). Intraoperative metabolic monitoring of the heart: I. Clinical assessment of coronary sinus metabolites. The Annals of Thoracic Surgery, 72(6):S2220-S2226. 
Dawson, G. D. (1947). Cerebral Responses to Electrical Stimulation of Peripheral Nerve in Man. Journal of neurology, neurosurgery, and psychiatry, 10(3):134-40.

Deletis, V. (2007). Basic methodological principles of multimodal intraoperative monitoring during spine surgeries. European spine journal : official publication of the European Spine Society, the European Spinal Deformity Society, and the European Section of the Cervical Spine Research Society, 16 Suppl 2:S147-52.

Deletis, V. and Sala, F. (2008). Intraoperative neurophysiological monitoring of the spinal cord during spinal cord and spine surgery: a review focus on the corticospinal tracts. Clinical neurophysiology : official journal of the International Federation of Clinical Neurophysiology, 119(2):248-64.

Delorme, A., Palmer, J., Onton, J., Oostenveld, R., and Makeig, S. (2012). Independent EEG sources are dipolar. PloS one, 7(2):e30135.

Dinner, D. S., Lüders, H., Lesser, R. P., Morris, H. H., Barnett, G., and Klem, G. (1986). Intraoperative spinal somatosensory evoked potential monitoring. Journal of neurosurgery, 65(6):807-14.

Epstein, N. E., Danto, J., and Nardi, D. (1993). Evaluation of Intraoperative Somatosensory-Evoked Potential Monitoring During 100 Cervical Operations. Spine, 18(6):737-747.

Friedman, W. A., Chadwick, G. M., Verhoeven, F. J., Mahla, M., and Day, A. L. (1991). Monitoring of somatosensory evoked potentials during surgery for middle cerebral artery aneurysms. Neurosurgery, 29(1):83-8.

Frigo, M. and Johnson, S. G. S. S. (1998). FFTW: an adaptive software architecture for the FFT. In Proceedings of the 1998 IEEE International Conference on Acoustics, Speech and Signal Processing, ICASSP '98 (Cat. No.98CH36181), volume 3, pages 1381-1384. IEEE.

Fukuda, H., Sonoo, M., and Ishibashi, M. (2007). Muscle afferent contributions to tibial nerve somatosensory evoked potentials investigated using knee stimulations. Clinical Neurophysiology, 118(9):2104-2111.

Glaser, E. and Ruchkin, D. S. (1976). Principles of Neurobiological Signal Analysis. Academic Press, New York.

Goryawala, M., Yaylali, I., Cabrerizo, M., Vedala, K., and Adjouadi, M. (2011). An effective novel patient specific Gaussian template based scheme for somatosensory 
evoked potential detection. In 2011 IEEE Signal Processing in Medicine and Biology Symposium (SPMB), pages 1-6, New York, NY. IEEE.

Goryawala, M., Yaylali, I., Cabrerizo, M., Vedala, K., and Adjouadi, M. (2012). An effective intra-operative neurophysiological monitoring scheme for aneurysm clipping and spinal fusion surgeries. Journal of neural engineering, 9(2):026021.

Grundy, B. L. (1983). Intraoperative monitoring of sensory-evoked potentials. Anesthesiology, 58(1):72-87.

Hargadine, J. R. and Snyder, E. (1982). Brain stem and somatosensory evoked potentials: application in the operating room and intensive care unit. Bulletin of the Los Angeles neurological societies, 47:62-75.

Hu, Y., Liu, H., and Luk, K. D. (2011a). Time-frequency analysis of somatosensory evoked potentials for intraoperative spinal cord monitoring. Journal of clinical neurophysiology : official publication of the American Electroencephalographic Society, 28(5):504-11.

Hu, Y., Liu, H., and Luk, K. D. (2011b). Time-frequency analysis of somatosensory evoked potentials for intraoperative spinal cord monitoring. Journal of clinical neurophysiology : official publication of the American Electroencephalographic Society, 28(5):504-11.

Hu, Y., Luk, K. D., and Xie, X. (2009). Surface somatosensory evoked potential detection by FPGA based multi-adaptive filter. In 2009 4th International IEEE/EMBS Conference on Neural Engineering, pages 673-676. IEEE.

Hu, Y., Luk, K. D. K., Lu, W. W., and Leong, J. C. Y. (2003). Application of timefrequency analysis to somatosensory evoked potential for intraoperative spinal cord monitoring. Journal of neurology, neurosurgery, and psychiatry, 74(1):82-7.

Hussain, A. M. (2008). A Practical Approach to Neurophysiologic Intraoperative Monitoring. Demos Medical Publishing.

Jones, S. J., Edgar, M. A., Ransford, A. O., and Thomas, N. P. (1983). A system for the electrophysiological monitoring of the spinal cord during operations for scoliosis. The Journal of bone and joint surgery. British volume, 65(2):134-9.

Jou, I.-M., Hsu, C.-C., Chern, T.-C., Chen, W.-Y., and Dau, Y.-C. (2003). Spinal somatosensory evoked potential evaluation of acute nerve-root injury associated with pedicle-screw placement procedures: an experimental study. Journal of 
orthopaedic research : official publication of the Orthopaedic Research Society, $21(2): 365-72$.

Kany, C. and Treede, R. D. (1997). Median and tibial nerve somatosensory evoked potentials: middle-latency components from the vicinity of the secondary somatosensory cortex in humans. Electroencephalography and Clinical Neurophysiology, 104(5):402-10.

Kaplan, A. Y., Fingelkurts, A. a., Fingelkurts, A. a., Borisov, S. V., and Darkhovsky, B. S. (2005). Nonstationary nature of the brain activity as revealed by EEG/MEG: Methodological, practical and conceptual challenges. Signal Processing, 85(11):2190-2212.

Khan, M. H., Smith, P. N., Balzer, J. R., Crammond, D., Welch, W. C., Gerszten, P., Sclabassi, R. J., Kang, J. D., and Donaldson, W. F. (2006). Intraoperative somatosensory evoked potential monitoring during cervical spine corpectomy surgery: experience with 508 cases. Spine, 31(4):E105-13.

Kilpatrick, C. C., Puig, C., Chohan, L., Monga, M., and Orejuela, F. J. (2010). Intraoperative fetal heart rate monitoring during nonobstetric surgery in pregnancy: a practice survey. Southern medical journal, 103(3):212-5.

Kong, X. and Oiu, T. (2001). Latency change estimation for evoked potentials: A comparison of algorithms. Medical \& Biological Engineering \& Computing, 39(2):208-224.

Lam, A. M., Manninen, P. H., Ferguson, G. G., and Nantau, W. (1991). Monitoring electrophysiologic function during carotid endarterectomy: a comparison of somatosensory evoked potentials and conventional electroencephalogram. Anesthesiology, 75(1):15-21.

Lam, B. S. C., Hu, Y., Lu, W. W., Luk, K. D. K., Chang, C. Q., Qiu, W., and Chan, F. H. Y. (2005). Multi-adaptive filtering technique for surface somatosensory evoked potentials processing. Medical engineering \& physics, 27(3):257-66.

Liu, H., Chang, C. Q., Luk, K. D. K., and Hu, Y. (2011). Comparison of blind source separation methods in fast somatosensory-evoked potential detection. Journal of clinical neurophysiology : official publication of the American Electroencephalographic Society, 28(2):170-7.

Liu, H., Hu, Y., Chang, C. Q., and Luk, K. D. K. (2007). Fast extraction of somatosensory evoked potential based on second order blind identification. In 
29th Annual International Conference of the IEEE Engineering in Medicine and Biology Society, volume 2007, pages 5457-60, Lyon. IEEE.

Loring, T. T. A., Worth, D. D. E., and Tang, A. A. C. (2004). Multiresolution metrics for detecting single-trial evoked response potentials (ERPS). In Proceedings of 2004 International Conference on Machine Learning and Cybernetics (IEEE Cat. No.04EX826), volume 7, pages 4240-4245. IEEE.

MacDonald, D. B., Al Zayed, Z., and Stigsby, B. (2005). Tibial somatosensory evoked potential intraoperative monitoring: recommendations based on signal to noise ratio analysis of popliteal fossa, optimized P37, standard P37, and P31 potentials. Clinical neurophysiology : official journal of the International Federation of Clinical Neurophysiology, 116(8):1858-69.

Madhok, J., Maybhate, A., Xiong, W., Koenig, M. A., Geocadin, R. G., Jia, X., and Thakor, N. V. (2010). Quantitative assessment of somatosensory-evoked potentials after cardiac arrest in rats: prognostication of functional outcomes. Critical care medicine, 38(8):1709-17.

Mahla, M. E., Long, D. M., McKennett, J., Green, C., and McPherson, R. W. (1984). Detection of brachial plexus dysfunction by somatosensory evoked potential monitoring-a report of two cases. Anesthesiology, 60(3):248-52.

McGarry, J., Friedgood, D. L., Woolsey, R., Horenstein, S., and Johnson, C. (1984). Somatosensory-evoked potentials in spinal cord injuries. Surgical neurology, 22(4):341-3.

Moore, B. (1981). Principal component analysis in linear systems: Controllability, observability, and model reduction. IEEE Transactions on Automatic Control, 26(1):17-32.

Nash, C. L., Lorig, R. a., Schatzinger, L. a., and Brown, R. H. (1977). Spinal cord monitoring during operative treatment of the spine. Clinical orthopaedics and related research, 126:100-5.

Nercessian, O. A., Gonzalez, E. G., and Stinchfield, F. E. (1989). The use of somatosensory evoked potential during revision or reoperation for total hip arthroplasty. Clinical orthopaedics and related research, (243):138-42.

Nishida, S., Nakamura, M., and Shibasaki, H. (1993). Method for single-trial recording of somatosensory evoked potentials. Journal of biomedical engineering, 15(3):257-62. 
Noss, R. S., Boles, C. D., and Yingling, C. D. (1996). Steady-state analysis of somatosensory evoked potentials. Electroencephalography and Clinical Neurophysiology, 100(5):453-61.

Nuwer, M. R. (2008). Intraoperative Monitoring of Neural Function. Elsevier Science Health Science Division.

Nuwer, M. R. and Dawson, E. (1984). Intraoperative evoked potential monitoring of the spinal cord: enhanced stability of cortical recordings. Electroencephalography and clinical neurophysiology, 59(4):318-27.

Nuwer, M. R., Dawson, E. G., Carlson, L. G., Kanim, L. E., and Sherman, J. E. (1995). Somatosensory evoked potential spinal cord monitoring reduces neurologic deficits after scoliosis surgery: results of a large multicenter survey. Electroencephalography and clinical neurophysiology, 96(1):6-11.

Pastorelli, F., Di Silvestre, M., Plasmati, R., Michelucci, R., Greggi, T., Morigi, A., Bacchin, M. R., Bonarelli, S., Cioni, A., Vommaro, F., Fini, N., Lolli, F., and Parisini, P. (2011). The prevention of neural complications in the surgical treatment of scoliosis: the role of the neurophysiological intraoperative monitoring. European spine journal : official publication of the European Spine Society, the European Spinal Deformity Society, and the European Section of the Cervical Spine Research Society, 20 Suppl 1:S105-14.

Porcaro, C., Coppola, G., Di Lorenzo, G., Zappasodi, F., Siracusano, A., Pierelli, F., Rossini, P. M., Tecchio, F., and Seri, S. (2009). Hand somatosensory subcortical and cortical sources assessed by functional source separation: an EEG study. Human brain mapping, 30(2):660-74.

Porter, S. S., Black, D. L., Reckling, F. W., and Mason, J. (1989). Intraoperative cortical somatosensory evoked potentials for detection of sciatic neuropathy during total hip arthroplasty. Journal of Clinical Anesthesia, 1(3):170-176.

Regan, D. (1990). Human Brain Electrophysiology: Evoked Potentials and Evoked Magnetic Fields in Science and Medicine, volume 74. Elsevier Science Publishing, New York.

Sako, K., Nakai, H., Kawata, Y., Takizawa, K., Satho, M., and Yonemasu, Y. (1998). Temporary arterial occlusion during anterior communicating or anterior cerebral artery aneurysm operation under tibial nerve somatosensory evoked potential monitoring. Surgical neurology, 49(3):316-22; discussion 322-3. 
Schwartz, D. M., Auerbach, J. D., Dormans, J. P., Flynn, J., Drummond, D. S., Bowe, J. A., Laufer, S., Shah, S. A., Bowen, J. R., Pizzutillo, P. D., and Jones, K. J. (2007). Neurophysiological detection of impending spinal cord injury during scoliosis surgery. The Journal of bone and joint surgery. American volume, $89(11): 2440-9$.

Simpson, D. M., Tierra-Criollo, C. J., Leite, R. T., Zayen, E. J., and Infantosi, A. F. (2000). Objective response detection in an electroencephalogram during somatosensory stimulation. Annals of Biomedical Engineering, 28(6):691-8.

Smith, W. D. (1981). Walsh versus Fourier estimators of the EEG power spectrum. IEEE Transactions on Biomedical Engineering, 28(11):790-3.

Society, A. C. N. (2006). Guideline 9A: Guidelines on evoked potentials. Journal of clinical neurophysiology : official publication of the American Electroencephalographic Society, 23(2):125-37.

Stoica, P. and Moses, R. L. (1997). Introduction to spectral analysis. Prentice Hall, Upper Saddle River, New Jersey, 1 edition.

Strahm, C., Min, K., Boos, N., Ruetsch, Y., and Curt, A. (2003). Reliability of perioperative SSEP recordings in spine surgery. Spinal cord : the official journal of the International Medical Society of Paraplegia, 41(9):483-9.

Suter, C. M. (1970). Principal component analysis of average evoked potentials. Experimental Neurology, 29(2):317-327.

Terada, K., Umeoka, S., Baba, K., Sakura, Y., Usui, N., Matsuda, K., Tottori, T., Mihara, T., Usui, K., Nakamura, F., and Inoue, Y. (2009). Generators of tibial nerve somatosensory evoked potential: recorded from the mesial surface of the human brain using subdural electrodes. Journal of Clinical Neurophysiology, 26(1):13-6.

Tito, M., Cabrerizo, M., Ayala, M., Jayakar, P., and Adjouadi, M. (2010). A comparative study of intracranial EEG files using nonlinear classification methods. Annals of biomedical engineering, 38(1):187-99.

Toleikis, J. R. (2005). Intraoperative monitoring using somatosensory evoked potentials. A position statement by the American Society of Neurophysiological Monitoring. Journal of clinical monitoring and computing, 19(3):241-58. 
Tong, L., Liu, R.-w., Soon, V., and Huang, Y.-F. (1991). Indeterminacy and identifiability of blind identification. IEEE Transactions on Circuits and Systems, 38(5):499-509.

Tong, L., Soon, V. V., Huang, Y., and Liu, R. (1990). AMUSE: a new blind identification algorithm. In IEEE International Symposium on Circuits and Systems, volume 3, pages 1784-1787. IEEE.

Truccolo, W., Knuth, K. H., Shah, A., Bressler, S. L., Schroeder, C. E., and Ding, M. (2003). Estimation of single-trial multicomponent ERPs: differentially variable component analysis (dVCA). Biological Cybernetics, 89(6):426-38.

Turetsky, B. I., Raz, J., and Fein, G. (1989). Estimation of trial-to-trial variation in evoked potential signals by smoothing across trials. Psychophysiology, 26(6):70012 .

van de Wassenberg, W. J. G., Kruizinga, W. J., van der Hoeven, J. H., Leenders, K. L., and Maurits, N. M. (2008). Multichannel recording of tibial-nerve somatosensory evoked potentials. Neurophysiologie clinique = Clinical neurophysiology, 38(5):277-88.

Vedala, K., Yaylali, I., Cabrerizo, M., Goryawala, M., and Adjouadi, M. (2012a). Intraoperative Neurophysiologic Monitoring During Surgical Treatment of Spinal Surgeries. In Neurocritical Care Society, 10th Annual Meeting, volume 17 Suppl 2, page S66, Denver, CO. Neurocritical Care.

Vedala, K., Yaylali, I., Cabrerizo, M., Goryawala, M., and Adjouadi, M. (2012b). Peak detection of somatosensory evoked potentials using an integrated principal component analysis-walsh method. Journal of clinical neurophysiology : official publication of the American Electroencephalographic Society, 29(2):165-73.

Weide, B. W., Andrews, L. T., and Iannone, A. M. (1978). Real-time analysis of EEG using Walsh transforms. Computers in Biology and Medicine, 8(4):255-263.

Wirfalt, P. and Jansson, M. (2010). On Toeplitz and Kronecker structured covariance matrix estimation. In 2010 IEEE Sensor Array and Multichannel Signal Processing Workshop, pages 185-188. IEEE.

Zhang, X.-S. S., Roy, R. J., Schwender, D., and Daunderer, M. (2001). Discrimination of Anesthetic States using Mid-Latency Auditory Evoked Potential and Artificial Neural Networks. Annals of Biomedical Engineering, 29(5):446-453. 
Zygierewicz, J., Mazurkiewicz, J., Durka, P. J., Franaszczuk, P. J., and Crone, N. E. (2006). Estimation of short-time cross-correlation between frequency bands of event related EEG. Journal of Neuroscience Methods, 157(2):294-302. 
VITA

KrishnateJ Vedala

2007

B.E., Electronics and Telecommunication

North Maharashtra University

Jalgaon, India

2008

M.S., Electrical Engineering

Florida International University

Miami, Florida

2013

Ph. D. Candidate, Electrical Engineering

Florida International University

Miami, Florida

\section{JOURNAL PUBLICATIONS}

Vedala K., Motahari A., Cabrerizo M., Goryawala M., Yaylali I. \& Adjouadi M. Quasi-stationaryity of EEG for Intraoperative Monitoring During Spinal Surgeries. The Scientific World Journal, Under review.

Goryawala, M., Yaylali, I., Cabrerizo, M., Vedala, K. \& Adjouadi, M. An effective intra-operative neurophysiological monitoring scheme for aneurysm clipping and spinal fusion surgeries. Journal of neural engineering 9(2), 026021, pp:1-12 (2012). PMID: 22419062

Vedala, K., Yaylali, I., Cabrerizo, M., Goryawala, M. \& Adjouadi, M. Peak detection of somatosensory evoked potentials using an integrated principal component analysis-walsh method. Journal of clinical neurophysiology : official publication of the American Electroencephalographic Society 29, pp:165-73 (2012). PMID: 22469683

\section{CONFERENCE PUBLICATIONS}

Vedala K., Motahari A., Goryawala M., Cabrerizo M., Yaylali I. \& Adjouadi M. Novel Time-Frequency-Eigen Filter for Intraoperative Neurophysiologic Monitoring in Spinal Surgeries. Neural Engineering (NER), 2013 6th International IEEE/EMBS Conference on, In Press. 
Motahari A., Vedala K., Goryawala M., Cabrerizo M., Yaylali I. \& Adjouadi M. A SomatoSensory Evoked Potential Monitoring Algorithm Using Time Frequency Filtering. Neural Engineering (NER), 2013 6th International IEEE/EMBS Conference on, In Press.

Vedala, K., Yaylali, I., Cabrerizo, M., Goryawala, M. \& Adjouadi, M. Intraoperative neurophysiologic monitoring during surgical treatment of spinal surgeries. In 2013 10th Annual Neurocritical Care Society Meeting, [Denver, CO - Oct. 5, 2012], Abstract in: Neurocritical Care, 17 Suppl 2, pp: S1-337 (2012). PMID: 22990584

Goryawala, M., Yaylali, I., Cabrerizo, M., Vedala, K. \& Adjouadi, M. An effective novel patient specific Gaussian template based scheme for somatosensory evoked potential detection. In 2011 IEEE Signal Processing in Medicine and Biology Symposium (SPMB) pp:1-6 (2011). PMID: 22419062 